\title{
Small Panchromatic and NIR Absorbers from Quinoid Zwitterions
}

\author{
Angélina Torres Ruiz, ${ }^{\dagger, \ddagger}$ Manon H. E. Bousquet,,$₫$ Simon Pascal, ${ }^{*}, \dagger$ Gabriel \\ Canard, ${ }^{\dagger}$ Valérie Mazan, Mourad Elhabiri, Denis Jacquemin, ${ }^{*}$, Olivier Siri*, $\dagger$ \\ † Aix-Marseille Université, CNRS UMR 7325, Centre Interdisciplinaire de Nanoscience de Marseille \\ (CINaM), Campus de Luminy, 13288 Marseille cedex 09, France. \\ $\S$ Laboratoire CEISAM, CNRS UMR 6230, Université de Nantes, 2, rue de la Houssinière, 44322 \\ Nantes, France. \\ "Université de Strasbourg, Université de Haute-Alsace, CNRS, LIMA, UMR 7042, Equipe Chimie \\ Bioorganique et Médicinale, ECPM, 25 Rue Becquerel, 67000 Strasbourg, France.
}


TABLE OF CONTENT

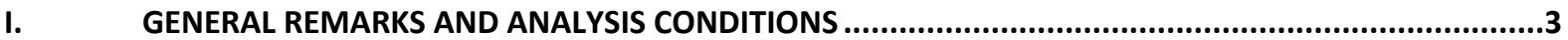

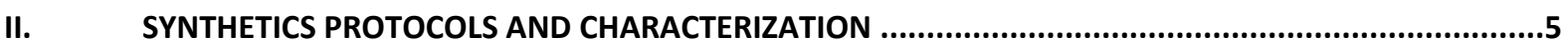

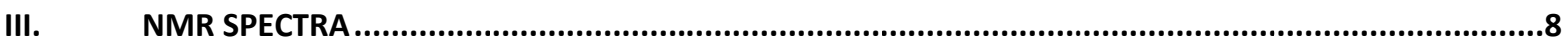

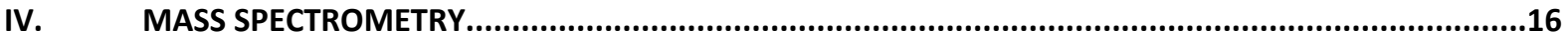

V. INFRARED SPECTROSCOPY

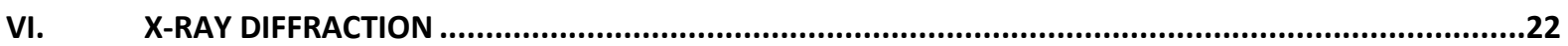

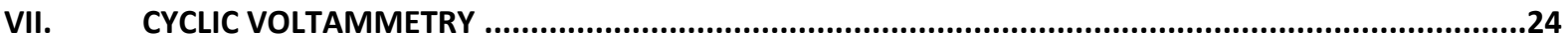

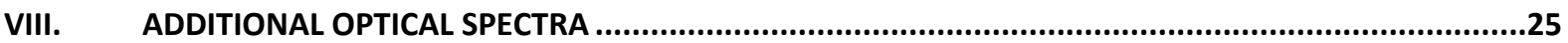

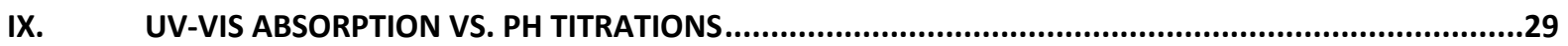

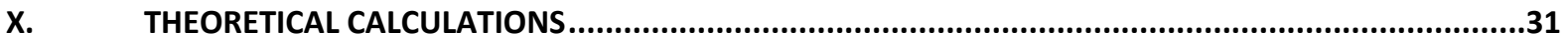

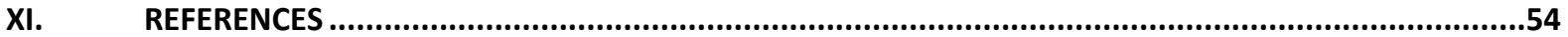




\section{GENERAL REMARKS AND ANALYSIS CONDITIONS}

Reagents. Unsubstituted zwitterion 1 was prepared from 4,6-diaminoresorcinol dihydrochloride. ${ }^{1}$ Compounds 2, 3a and $\mathbf{3 b}$ were prepared according to reported procedures. ${ }^{2}$ 4,6-diaminoresorcinol dihydrochloride was purchased from Sigma Aldrich. When heating was required, oil bathes were used.

Analytical methods and apparatus. Melting points (M.P.) were measured in open capillary tubes with a STUART SMP30 melting points apparatus and are uncorrected. NMR spectra were recorded JEOL ECS400 NMR spectrometer at room temperature (otherwise noted). NMR chemical shifts are given in ppm (ס) relative to $\mathrm{Me}_{4} \mathrm{Si}$ with solvent resonances used as internal standards $\left(\mathrm{CDCl}_{3}: 7.26 \mathrm{ppm}\right.$ for ${ }^{1} \mathrm{H}$ and 77.2 for ${ }^{13} \mathrm{C} ; \mathrm{CD}_{2} \mathrm{Cl}_{2}: 5.32 \mathrm{ppm}$ for ${ }^{1} \mathrm{H}$ and 53.8 for ${ }^{13} \mathrm{C}$; Acetone- $d_{6}: 2.05 \mathrm{ppm}$ for ${ }^{1} \mathrm{H}$ and 29.8 for ${ }^{13} \mathrm{C}$; DMSO- $d_{6}$ : 2.50 ppm for ${ }^{1} \mathrm{H}$ and 39.5 for ${ }^{13} \mathrm{C}$ ). IR spectra were recorded on an Agilent Cary 630 FTIR equipped with an attenuated total reflectance (ATR) sampling. HRMS analyses were performed on a QStar Elite (Applied Biosystems SCIEX) or a SYNAPT G2 HDMS (Waters) spectrometers by the "Spectropole" of the Aix-Marseille University. These two instruments were equipped with an ESI or MALDI source, and a TOF mass analyzer.

Crystallography. Suitable crystal for compound $\mathbf{2}$ was obtained from slow diffusion from a solution of the compound in acetone to pentane. It was mounted on a Rigaku Oxford Diffraction SuperNova diffractometer and measured at $293 \mathrm{~K}$ at the $\mathrm{Cu}$ radiation $(\lambda=1.54184 \AA)$. Data collection, reduction and multiscan ABSPACK correction were performed with CrysAlisPro (Rigaku Oxford Diffraction). Using

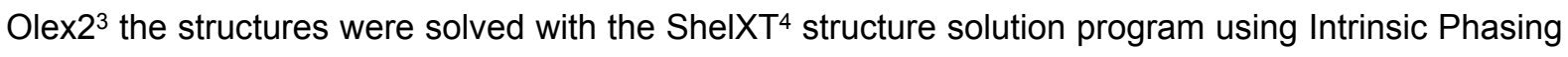
and refined with ShelXL ${ }^{4}$ using least-square minimization.

Electrochemistry. Cyclic voltammetry (CV) data were recorded using a BAS 100 (Bioanalytical Systems) potentiostat and the BAS100W software (v2.3). All the experiments were conducted under an argon atmosphere in a standard one-compartment using a three electrodes setup: a Pt working electrode $(\varnothing=1.6 \mathrm{~mm}$ ), a Pt counter electrode and an $\mathrm{Ag} / \mathrm{AgCl}$ reference electrode (filled with a $3 \mathrm{M}$ $\mathrm{NaCl}$ solution). Tetra- $n$-butylammonium hexafluorophosphate $\left(\left[\mathrm{TBA}^{\mathrm{B}}\left[\mathrm{PF}_{6}\right]\right)\right.$ was used as supporting electrolytes $\left(10^{-1} \mathrm{M}\right)$, with a concentration of the electro-active compound ca. $10^{-3} \mathrm{M}$ in DCM. The reference electrode was calibrated using ferrocene $\left(\mathrm{E}^{\circ}\left(\mathrm{Fc} / \mathrm{Fc}^{+}\right)=0.46 \mathrm{~V} / \mathrm{SCE}(\mathrm{DCM})\right) .{ }^{5}$ The scan rate was $100 \mathrm{mV} / \mathrm{S}$. Before experiment, the solution was degassed using argon and the working electrode (Pt) was polished before each recording.

Electronic absorption. UV-vis-NIR absorption spectra were recorded in spectrophotometric grade solvents (ca. 10-5 M) on a VARIAN CARY 50 SCAN spectrophotometer at room temperature with a 300 $\mathrm{nm} / \mathrm{min}$ scan rate.

Absorption spectrophotometric titrations versus $\mathrm{pH}$. Distilled water was purified by passing it through a mixed bed of ion-exchanger (Bioblock Scientific R3-83002, M3-83006) and activated carbon (Bioblock Scientific ORC-83005). Spectrophotometric grade methanol (Merck, p.a.) was used and water was de-oxygenated by $\mathrm{CO}_{2}$ - and $\mathrm{O}_{2}$-free argon (Sigma Oxiclear cartridge) before use. All the stock solutions were prepared by weighing solid products using an AG 245 Mettler Toledo analytical balance 
(precision $0.01 \mathrm{mg})$. The ionic strength was maintained at $0.1 \mathrm{M}$ with either sodium chloride $(\mathrm{NaCl}$, Carlo-Erba-SDS Phar. Eur. 99-100.5\%) for titrations in water/DMSO 90/10 w/w or tetrabutylammonium perchlorate $\left(n \mathrm{NBu}_{4} \mathrm{ClO}_{4}\right.$, Sigma-Aldrich, $\left.>98 \%\right)$ for titrations in methanol/water $80 / 20 \mathrm{w} / \mathrm{w}$, and all measurements were carried out at 25.0(2) $\left.{ }^{\circ} \mathrm{C}\right)$. CAUTION! Perchlorate salts combined with organic ligands are potentially explosive and should be handled in small quantities and with the adequate precautions. Stock solutions of the BQI derivatives $3 \mathbf{a}\left(4.75 \times 10^{-3} \mathrm{M}\right)$ and $3 \mathrm{~d}\left(3.27 \times 10^{-3} \mathrm{M}\right.$ and $4.09 \mathrm{x}$ $10^{-3} \mathrm{M}$ ) were first prepared by quantitative dissolution of the corresponding solid samples in methanol. The methanolic stock solutions were then diluted in $\mathrm{CH}_{3} \mathrm{OH} / \mathrm{H}_{2} \mathrm{O}$ solvent $(80 / 20 \mathrm{w} / \mathrm{w})$ containing the supporting electrolyte at $0.1 \mathrm{M}\left(n \mathrm{NBu}_{4} \mathrm{ClO}_{4}\right)$. An aliquot of $40 \mathrm{~mL}$ of $3 \mathrm{a}\left(4.66 \times 10^{-5} \mathrm{M}\right)$ or $3 \mathrm{~d}\left(3.96 \times 10^{-5}\right.$ $\mathrm{M}$ for $5.68<\mathrm{pH}<11.73$ and $4.03 \times 10^{-5} \mathrm{M}$ for $2.27<\mathrm{pH}<5.05$ ) solution was then introduced into a jacketed cell (Metrohm) maintained at $25.0 \pm 0.2^{\circ} \mathrm{C}$ by the flow of a Lauda E200 thermostat. The free hydrogen ion concentration was measured with a combined glass electrode (Metrohm 6.0234.500, Long Life; $\mathrm{Ag} / \mathrm{AgCl}$ reference glass electrode filled with $0.1 \mathrm{M} \mathrm{NaCl}$ in $\mathrm{CH}_{3} \mathrm{OH} / \mathrm{H}_{2} \mathrm{O} 80 / 20 \mathrm{w} / \mathrm{w}$ ) and an automatic titrator system 794 Basic Titrino (Metrohm) connected to a microcomputer (Tiamo light 1.2 program). The combined glass electrode was calibrated as a hydrogen concentration probe by titrating known amounts of perchloric acid $\left(0.126 \mathrm{M}\right.$ from $\mathrm{HClO}_{4}$, Prolabo, normapur, $\left.70 \% \mathrm{~min}\right)$ with $\mathrm{CO}_{2}$-free tetrabutylammonium hydroxide $n \mathrm{NBu}_{4} \mathrm{OH}$ solution $\left(0.097 \mathrm{M}\right.$ from Fluka, $\sim 40 \%$ in water). ${ }^{6}$ The $\mathrm{HClO}_{4}$ and $n-\mathrm{NBu}_{4} \mathrm{OH}$ solutions were freshly prepared just before use in $\mathrm{CH}_{3} \mathrm{OH} / \mathrm{H}_{2} \mathrm{O}$ solvent and their analytical concentrations were ascertained by colorimetric titrations with sodium tetraborate decahydrate $\left(\mathrm{B}_{4} \mathrm{Na}_{2} \mathrm{O}_{7} .10 \mathrm{H}_{2} \mathrm{O}\right.$, Fluka, puriss, p.a. $)$ and potassium hydrogen phthalate $\left(\mathrm{C}_{8} \mathrm{H}_{5} \mathrm{KO}_{3}\right.$, Fluka, puriss, p.a.), respectively, using methyl orange (RAL) and phenolphthalein (Prolabo, purum) as the indicators. The initial $\mathrm{pH}$ of the $\mathrm{BQI}$ solutions were adjusted to $\sim 2-3$ with $\mathrm{HClO}_{4}$ (Prolabo, normapur, $70 \% \mathrm{~min}$ ), and the titrations (3a: $3.09<\mathrm{pH}<11.50$ and $3 \mathrm{~d}$ : $2.27<\mathrm{pH}<5.05$ and $5.68<\mathrm{pH}<11.73$ ) were then carried out by addition of known volumes of $n-\mathrm{NBu}_{4} \mathrm{OH}$ solutions using the automatic titrator of the 794 Basic Titrino device (Metrohm). After each addition (i.e., DET - dynamic Potential Titration method of the Tiamo program used with a measuring point density of 3), an absorption spectra was recorded using a Varian CARY 50 spectrophotometer fitted with Hellma optical fibers (Hellma, 041.002UV) and an immersion probe made of quartz suprasil (Hellma, 661.500-QX) and interfaced (Cetrib) with the potentiometric unit. The same experimental procedure was used to determine the protonation properties of compound $3 a$ in a 90/10 $w / w$ water/DMSO mixture $\left(p K_{a}=8.64 \pm 0.05\right)$ but could not be applied to $\mathbf{3 d}$ for solubility reasons.

The spectrophotometric data were analyzed with Specfit ${ }^{7-9}$ program which adjusts the absorptivities and the stability constants of the species formed at equilibrium. Specfit uses factor analysis to reduce the absorbance matrix and to extract the eigenvalues prior to the multiwavelength fit of the reduced data set according to the Marquardt algorithm. ${ }^{10,11}$ 


\section{SYNTHETICS PROTOCOLS AND CHARACTERIZATION}

\section{Compound 3c}<smiles></smiles>

$3 c$

Under inert atmosphere, 1,4-phenylenediamine dihydrochloride $(11.86 \mathrm{~g}, 65.16$ mmol, 30 equiv.) and DIPEA (22.55 mL, $130.32 \mathrm{mmol}, 60$ equiv.) were added to a suspension of zwitterion 1 ( $300 \mathrm{mg}, 2.17 \mathrm{mmol}, 1$ equiv.) in absolute ethanol (50 $\mathrm{mL}$ ). The reaction mixture was heated to reflux for 4 days. The formed precipitate was filtered on a sintered glass and the crude solid was washed with ethyl acetate, $\mathrm{DCM}$, acetone, and $\mathrm{Et}_{2} \mathrm{O}$ to afford the product as a black solid (194 $\mathrm{mg}, 28 \%$ yield). M.P: > $300{ }^{\circ} \mathrm{C}$ (decomposition). ${ }^{1} \mathrm{H}$ NMR (400 MHz, DMSO-d6): $\delta=10.42$ (br s, 2H), $7.04(\mathrm{~d}, 4 \mathrm{H}, \mathrm{J}=7.71 \mathrm{~Hz}), 6.54(\mathrm{~d}, 4 \mathrm{H}, \mathrm{J}=7.71 \mathrm{~Hz}), 5.87(\mathrm{~s}, 1 \mathrm{H}), 5.42(\mathrm{br} \mathrm{s}, 4 \mathrm{H}), 5.11(\mathrm{~s}, 1 \mathrm{H})$. ${ }^{13} \mathrm{C}\left\{{ }^{1} \mathrm{H}\right\}$ NMR (101 MHz, DMSO-d6): $\delta=172.4\left(\mathrm{C}_{\text {quat }}\right), 153.3\left(\mathrm{C}_{\text {quat }}\right), 148.2\left(\mathrm{C}_{\text {quat }}\right), 125.3(\mathrm{CH}), 124.6$ ( $\left.\mathrm{C}_{\text {quat }}\right), 113.7(\mathrm{CH}), 98.1(\mathrm{CH}), 84.9(\mathrm{CH})$. UV-Vis: $\lambda_{\max }(\mathrm{DMSO})=594 \mathrm{~nm}\left(\varepsilon=3340 \mathrm{~L} \cdot \mathrm{mol}^{-1} \cdot \mathrm{cm}^{-1}\right)$. IR (neat, $\left.\mathbf{c m}^{-1}\right): v=3448,3417,3167,1629,1604,1540,1507,1473,1437,1348,1282,1239,1201$, 1167, 1131, 945, 906, 867, 821, 756, 710. HRMS (ESI) m/z: [M+ H] $]^{+}$Calcd for $\mathrm{C}_{18} \mathrm{H}_{17} \mathrm{~N}_{4} \mathrm{O}_{2}{ }^{+} 321.1346$; Found 321.1344.

\section{Compound 3d}<smiles></smiles>

$3 d$

$\mathrm{N}, \mathrm{N}$-Dimethyl-1,4-phenylenediamine $(7.4 \mathrm{~g}, 54.3 \mathrm{mmol}, 30$ equiv.) was added to a suspension of zwitterion 1 ( $250 \mathrm{mg}, 1.81 \mathrm{mmol}, 1$ equiv.) in absolute ethanol (25 $\mathrm{mL})$. The reaction mixture was heated to reflux for 3 days. The formed precipitate was filtered on a sintered glass and the crude solid was washed with acetone, DCM, $\mathrm{MeOH}$, diethyl ether and pentane. The product was obtained as a black solid (120 mg, $18 \%$ yield). M.P: $267-269{ }^{\circ} \mathrm{C} .{ }^{1} \mathrm{H}$ NMR (400 MHz, DMSO-d6): $\delta=10.57$ (s, $2 \mathrm{H}), 7.24(\mathrm{~d}, 4 \mathrm{H}, \mathrm{J}=8.42 \mathrm{~Hz}), 6.74(\mathrm{~d}, 4 \mathrm{H} \mathrm{J}=8.48 \mathrm{~Hz}), 5.93(\mathrm{~s}, 1 \mathrm{H}), 5.13(\mathrm{~s}, 1 \mathrm{H})$, $2.91(\mathrm{~s}, 12 \mathrm{H})$. No ${ }^{13} \mathrm{C}$ NMR spectrum could be recorded due to poorly resolved signals and poor solubility. UV-Vis: $\lambda_{\max }(\mathrm{DMSO})=643 \mathrm{~nm}\left(\varepsilon=3087 \mathrm{~L} \cdot \mathrm{mol}^{-1} \cdot \mathrm{cm}^{-1}\right)$. IR (neat, $\left.\mathbf{c m}^{-1}\right): \mathrm{v}=$ 3292, 2881, 2796, 1647, 1596, 1574, 1560, 1497, 1438, 1420, 1350, 1311, 1216, 1159, 1119, 1073 , 1056, 995, 943, 814, 747. HRMS (ESI) m/z: [M + H] $]^{+}$Calcd for $\mathrm{C}_{22} \mathrm{H}_{25} \mathrm{~N}_{4} \mathrm{O}_{2}{ }^{+} 377.1972$; Found 377.1972. 


\section{Compound 4}

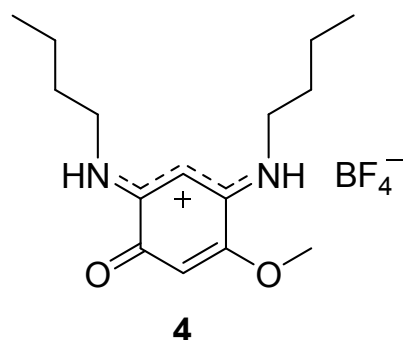

Compound 2 (50 mg, $0.19 \mathrm{mmol}, 1$ equiv.) was dissolved in anhydrous DCM $(10 \mathrm{~mL})$, and trimethyloxonium tetrafluoroborate $(29 \mathrm{mg}, 0.19 \mathrm{mmol}$, 1 equiv.) was added under inert atmosphere. After the solution was stirred at room temperature for $2.5 \mathrm{~h}$, the solvent was evaporated and the alkylated product was obtained as a red powder following precipitation in pentane (35 mg, 50\% yield). M.P: $100-102{ }^{\circ} \mathrm{C} .{ }^{1} \mathrm{H}$ NMR (400 $\mathbf{M H z}, \mathrm{CDCl}_{3}$ ): $\delta=9.43(\mathrm{br}, 2 \mathrm{H}), 5.84(\mathrm{~s}, 1 \mathrm{H}), 5.63(\mathrm{~s}, 1 \mathrm{H}), 4.05(\mathrm{~s}, 3 \mathrm{H}), 3.68(\mathrm{~m}, 2 \mathrm{H}), 3.48(\mathrm{~m}, 2 \mathrm{H}), 1.77(\mathrm{~m}, 4 \mathrm{H}), 1.46$ $(\mathrm{m}, 4 \mathrm{H}), 0.98(\mathrm{~m}, 6 \mathrm{H}) \cdot{ }^{13} \mathrm{C}\left\{{ }^{1} \mathrm{H}\right\} \operatorname{NMR}\left(101 \mathrm{MHz}, \mathrm{CDCl}_{3}\right): \delta=178.6$ ( $\left.\mathrm{C}_{\text {quat }}\right), 161.1$ ( $\left.\mathrm{C}_{\text {quat }}\right), 155.6$ ( $\left.\mathrm{C}_{\text {quat }}\right)$, 149.3 ( $\left.\mathrm{C}_{\text {quat }}\right), 102.9(\mathrm{CH}), 84.4(\mathrm{CH}), 58.4\left(\mathrm{CH}_{3}\right), 45.7\left(\mathrm{CH}_{2}\right), 44.0\left(\mathrm{CH}_{2}\right), 30.3\left(\mathrm{CH}_{2}\right), 30.2\left(\mathrm{CH}_{2}\right), 20.3$ $\left(\mathrm{CH}_{2}\right), 20.2\left(\mathrm{CH}_{2}\right), 13.8\left(\mathrm{CH}_{3}\right)$. UV-Vis: $\lambda_{\max }(\mathrm{DCM})=497 \mathrm{~nm}\left(\varepsilon=2249 \mathrm{~L} \cdot \mathrm{mol}^{-1} \cdot \mathrm{cm}^{-1}\right) \cdot{ }^{19} \mathrm{~F}$ NMR (376 MHz, $\mathbf{C D C l}_{3}$ ): $\delta=-152.6$. IR (neat, $\mathbf{c m}^{-1}$ ): $v=3254,3090,2962,2934,2875,1674,1606,1556,1515$, 1458, 1376, 1343, 1290, 1231, 1205, 1143, 1047, 1017, 985, 869, 788, 751, 718, 660. HRMS (ESI) m/z: [M] ${ }^{+}$Calcd for $\mathrm{C}_{15} \mathrm{H}_{25} \mathrm{~N}_{2} \mathrm{O}_{2}{ }^{+}$265.1911; Found 265.1913.

\section{Compound 5a}<smiles>COC1=CC(=O)/C(=N/c2ccccc2)C=C1Nc1ccccc1</smiles>

$5 \mathbf{a}$

Compound 3a (30 mg, $0.1 \mathrm{mmol}$ ) was dissolved in DCM, and trimethyloxonium tetrafluoroborate $(15.3 \mathrm{mg}, 0.1 \mathrm{mmol})$ was added to the solution. After the solution was stirred at $25{ }^{\circ} \mathrm{C}$ for $4 \mathrm{~h}$, the solvent was evaporated. The crude solution was filtered through a sintered glass with chloroform and after evaporation of the filtrate, the alkylated product was obtained as a purple powder by precipitation in $n$-hexane in a $72 \%$ yield $(29$ mg). M.P: $186-188^{\circ} \mathrm{C} .{ }^{1} \mathrm{H}$ NMR (400 MHz, Acetone-d $\left.{ }_{6}\right): \delta=11.44(\mathrm{br}, 1 \mathrm{H}), 10.59(\mathrm{br}, 1 \mathrm{H}), 7.58(\mathrm{~m}$, $5 \mathrm{H}), 7.52(\mathrm{~m}, 5 \mathrm{H}), 6.35(\mathrm{~s}, 1 \mathrm{H}), 6.29(\mathrm{~s}, 1 \mathrm{H}), 4.19(\mathrm{~s}, 3 \mathrm{H}) \cdot{ }^{13} \mathrm{C}\left\{{ }^{1} \mathrm{H}\right\}$ NMR (101 MHz, Acetone-d $\left.d_{6}\right): \delta=$ 180.2 ( $\left.\mathrm{C}_{\text {quat }}\right), 161.7\left(\mathrm{C}_{\text {quat }}\right), 157.1\left(\mathrm{C}_{\text {quat }}\right), 150.0\left(\mathrm{C}_{\text {quat }}\right), 136.6\left(\mathrm{C}_{\text {quat }}\right), 136.2\left(\mathrm{C}_{\text {quat }}\right), 130.9(\mathrm{CH}), 130.7$ $(\mathrm{CH}), 130.4(\mathrm{CH}), 129.6(\mathrm{CH}), 126.4(\mathrm{CH}), 125.6(\mathrm{CH}), 104.8(\mathrm{CH}), 89.4(\mathrm{CH}), 58.9\left(\mathrm{CH}_{3}\right) .{ }^{19} \mathrm{~F}$ NMR (376 MHz, Acetone-d $\mathrm{d}_{6}$ ): $\delta=-151.6$. UV-Vis: $\lambda_{\max }(\mathrm{DMSO})=502 \mathrm{~nm}\left(\varepsilon=4400 \mathrm{~L}^{\mathrm{mol}}{ }^{-1} \cdot \mathrm{cm}^{-1}\right)$. IR (neat, $\left.\mathbf{c m}^{-1}\right): \mathrm{v}=3065,2925,2854,1731,1663,1593,1540,1517,1488,1451,1373,1341,1282,1237,1207$, 1181, 1051, 998, 841, 758, 690, 664. HRMS (ESI) m/z: [M] Calcd for $\mathrm{C}_{19} \mathrm{H}_{17} \mathrm{~N}_{2} \mathrm{O}_{2}{ }^{+}$305.1285; Found 305.1284 . 


\section{Compound 5b}<smiles>COC1=CC(=O)C(Nc2ccc(OC)cc2)=CC1=Nc1ccc(OC)cc1</smiles>

$5 b$

Compound 3b (20 mg, $0.057 \mathrm{mmol}, 1$ equiv.) was dissolved in anhydrous $\mathrm{DCM}(5 \mathrm{~mL})$ and trimethyloxonium tetrafluoroborate $(8.4 \mathrm{mg}, 0.057 \mathrm{mmol}, 1$ equiv.) was added to the solution. After the reaction mixture was stirred at 25 ${ }^{\circ} \mathrm{C}$ for $3 \mathrm{~h}$, the solvent was evaporated and the crude residue was washed with $n$-hexane to afford the product as a green powder (19 $\mathrm{mg}, 73 \%$ yield). M.P: $221-223{ }^{\circ} \mathrm{C} .{ }^{1} \mathrm{H}$ NMR (400 MHz, DMSO-d 6 ): $\delta=11.11$ (br s, $2 \mathrm{H}$ ), 7.29 $(\mathrm{m}, 4 \mathrm{H}, \mathrm{J}=8.67 \mathrm{~Hz}, 17.77 \mathrm{~Hz}), 7.06(\mathrm{~m}, 4 \mathrm{H}, \mathrm{J}=8.62 \mathrm{~Hz}, 25.75 \mathrm{~Hz}), 6.30$ (s, 1H), $5.89(\mathrm{~s}, 1 \mathrm{H}), 4.02(\mathrm{~s}, 3 \mathrm{H}), 3.75(\mathrm{~s}, 3 \mathrm{H}), 3.72(\mathrm{~s}, 3 \mathrm{H}) .{ }^{13} \mathrm{C}\left\{{ }^{1} \mathrm{H}\right\}$ NMR $(101$

MHz, DMSO-d $\left.)_{6}\right): \delta=180.1$ ( $\left.\mathrm{C}_{\text {quat }}\right), 160.9\left(\mathrm{C}_{\text {quat }}\right), 159.1$ ( $\left.\mathrm{C}_{\text {quat }}\right), 158.6\left(\mathrm{C}_{\text {quat }}\right), 153.6\left(\mathrm{C}_{\text {quat }}\right), 147.6$ ( $\left.\mathrm{C}_{\text {quat }}\right)$, $128.8\left(\mathrm{C}_{\text {quat }}\right), 126.6(\mathrm{CH}), 125.8(\mathrm{CH}), 114.8(\mathrm{CH}), 114.7(\mathrm{CH}), 104.1(\mathrm{CH}), 88.3(\mathrm{CH}), 58.2\left(\mathrm{CH}_{3}\right), 55.6$ $\left(\mathrm{CH}_{3}\right), 55.5\left(\mathrm{CH}_{3}\right)$. One $\mathrm{C}_{\text {quat }}$ signal is missing, even after overnight ${ }^{13} \mathrm{C}$ NMR analysis. ${ }^{19} \mathrm{~F}$ NMR (376 MHz, DMSO-d $\left.{ }_{6}\right): \delta=-148.2$. UV-Vis: $\lambda_{\max }(\mathrm{DMSO})=538 \mathrm{~nm}\left(\varepsilon=6018 \mathrm{~L} \cdot \mathrm{mol}^{-1} \cdot \mathrm{cm}^{-1}\right)$. IR (neat, $\left.\mathrm{cm}^{-1}\right)$ : $v=3215,2908,2836,1663,1605,1591,1541,1509,1497,1458,1438,1379,1341,1306,1289,1257$, 1231, 1211, 1185, 1170, 1048, 1017, 958, 910, 958, 910, 781, 743, 711, 689. HRMS (ESI) m/z: [M] $]^{+}$ Calcd for $\mathrm{C}_{21} \mathrm{H}_{21} \mathrm{~N}_{2} \mathrm{O}_{4}{ }^{+}$365.1496; Found 365.1494.

\section{Compound 6}<smiles>CN(C)c1ccc(N=C2C=C(Nc3ccc(N(C)C)cc3)C(O)=C(F)C2=O)cc1</smiles>

6
Compound 3d (50 mg, $0.13 \mathrm{mmol}, 1$ equiv.) was dissolved in anhydrous DMSO $(10 \mathrm{~mL})$ and trimethyloxonium tetrafluoroborate $(20 \mathrm{mg}, 0.13 \mathrm{mmol}, 1$ equiv.) was added to the solution under inert atmosphere. After the reaction was stirred at $25^{\circ} \mathrm{C}$ for 4 days, the mixture was poured in water and washed with DCM and ethyl acetate. A solution of potassium hexafluorophosphate $(0.1 \mathrm{M})$ was added to the water layer and the product was extracted with DCM. The organic layer was washed once with water, dried over sodium sulfate, filtrated and evaporated under reduced pressure. The product was finally precipitated in diethyl ether to obtain a purple powder $\left(12 \mathrm{mg}, 17 \%\right.$ yield). M.P: $217-219{ }^{\circ} \mathrm{C} .{ }^{1} \mathbf{H}$ NMR (400 MHz, $\left.\mathrm{CD}_{2} \mathrm{Cl}_{2}\right): \delta=9.48(\mathrm{br} \mathrm{s}, 2 \mathrm{H}), 7.19(\mathrm{~d}, 4 \mathrm{H}, \mathrm{J}=8.88 \mathrm{~Hz}), 6.74(\mathrm{~d}, 4 \mathrm{H}, \mathrm{J}=8.88 \mathrm{~Hz}), 6.39$ $(\mathrm{s}, 1 \mathrm{H}), 3.01(\mathrm{~s}, 12 \mathrm{H}), 2.55(\mathrm{~s}, 3 \mathrm{H}) .{ }^{13} \mathrm{C}\left\{{ }^{1} \mathrm{H}\right\} \mathrm{NMR}\left(101 \mathrm{MHz}, \mathrm{CD}_{2} \mathrm{Cl}_{2}\right): \delta=150.60\left(\mathrm{C}_{\text {quat }}\right), 124.82(\mathrm{CH})$, 124.31 ( $\left.\mathrm{C}_{\text {quat }}\right), 115.03\left(\mathrm{C}_{\text {quat }}\right), 112.98(\mathrm{CH}), 85.70(\mathrm{CH}), 41.57\left(\mathrm{CH}_{3}\right), 40.60\left(\mathrm{CH}_{3}\right)$. Two $\mathrm{C}_{\text {quat }}$ signals are missing, even after overnight ${ }^{13} \mathrm{C}$ NMR analysis. ${ }^{19} \mathrm{~F}$ NMR (376 $\mathbf{M H z}, \mathrm{CD}_{2} \mathrm{Cl}_{2}$ ): $\delta=-72.0,-73.9$. UV-Vis: $\lambda_{\max }(\mathrm{DMSO})=674 \mathrm{~nm}\left(\varepsilon=2308 \mathrm{~L} \cdot \mathrm{mol}^{-1} \cdot \mathrm{cm}^{-1}\right) \cdot \mathbf{I R}$ (neat, $\left.\mathbf{c m}^{-1}\right): \mathrm{v}=3209,2854,2068,1603,1559$, 1508, 1356, 1227, 1166, 1123, 1021, 1000, 945, 837, 813. HRMS (ESI) m/z: [M] ${ }^{+}$Calcd for $\mathrm{C}_{23} \mathrm{H}_{27} \mathrm{~N}_{4} \mathrm{O}_{2}{ }^{+}$ 391.2129; Found 391.2126. 


\section{NMR SPECTRA}

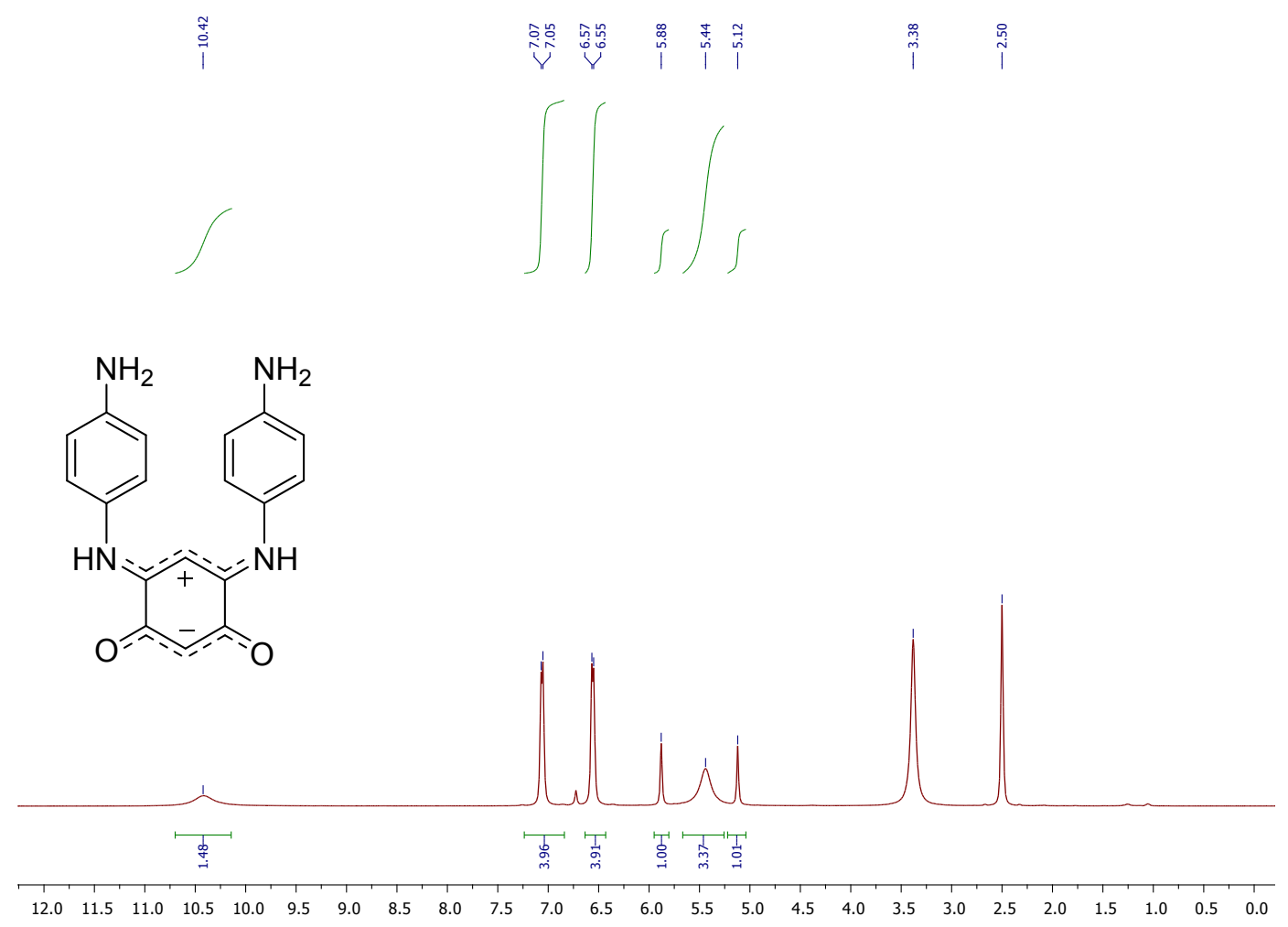

Figure S $1 .{ }^{1} \mathrm{H}$ NMR (400 MHz, DMSO- $\left.d_{6}\right)$ of compound $3 \mathbf{c}$.

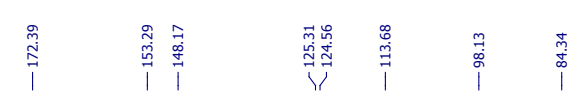<smiles></smiles>

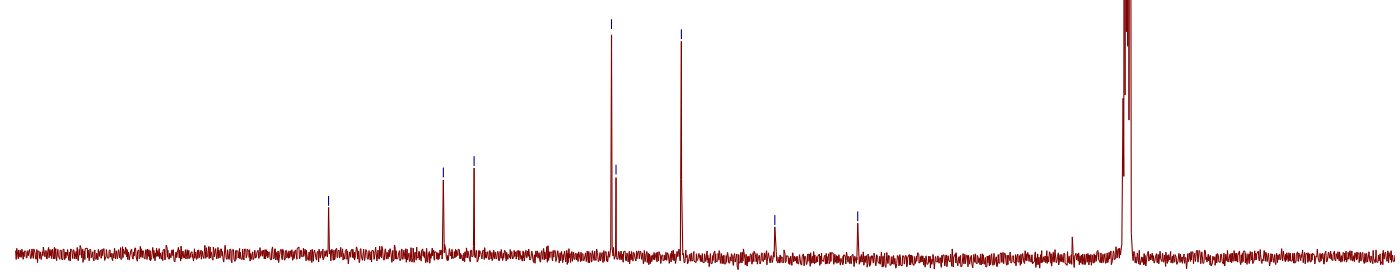

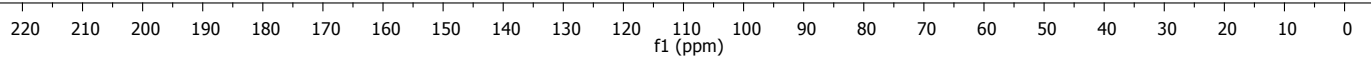

Figure S $2 .{ }^{13} \mathrm{C}$ NMR $\left(101 \mathrm{MHz}\right.$, DMSO- $\left.d_{6}\right)$ of compound $\mathbf{3 c}$. 


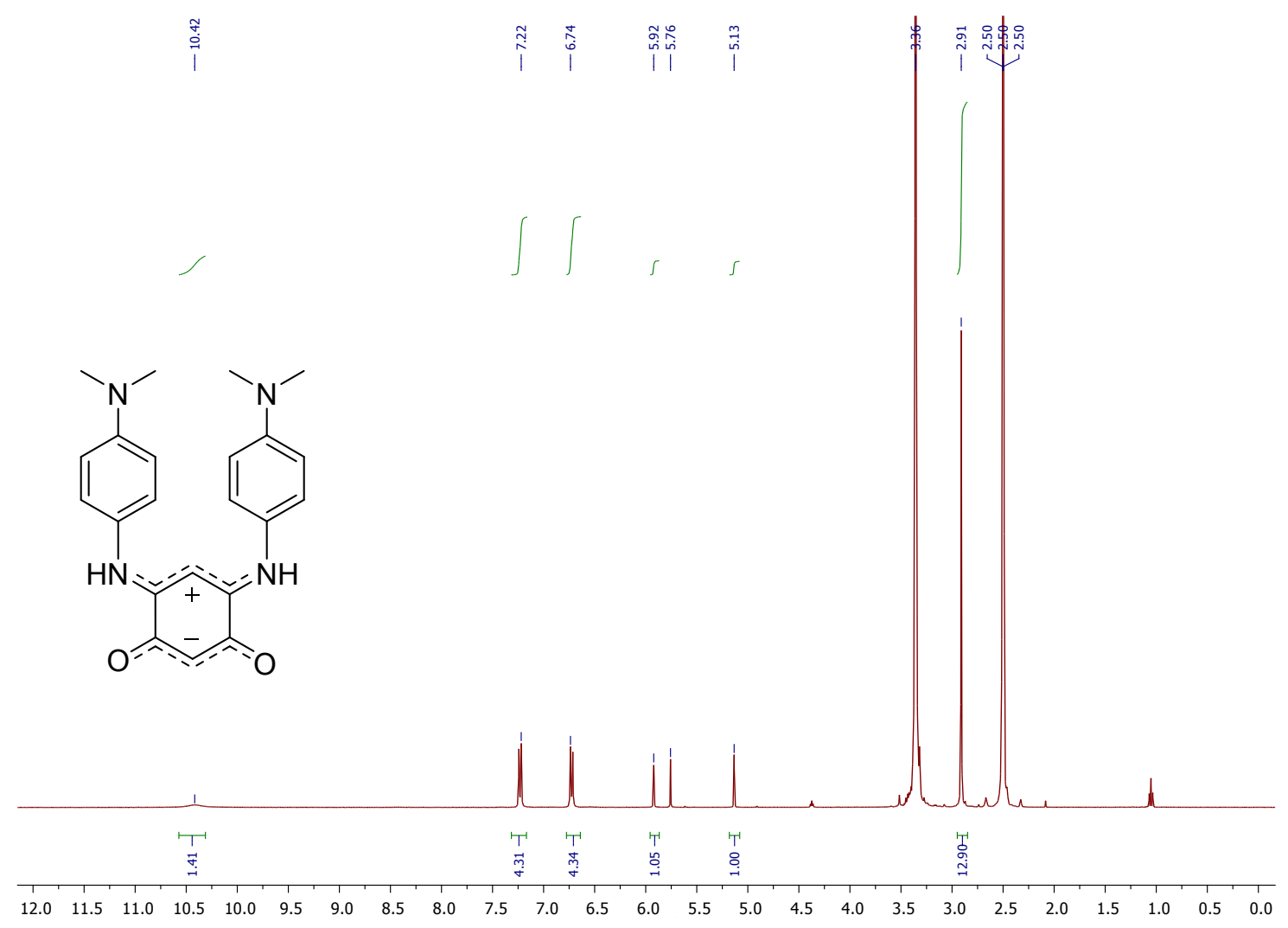

Figure S 3. ${ }^{1} \mathrm{H}$ NMR (400 MHz, DMSO- $d_{6}$ ) of compound $\mathbf{3 d}$.

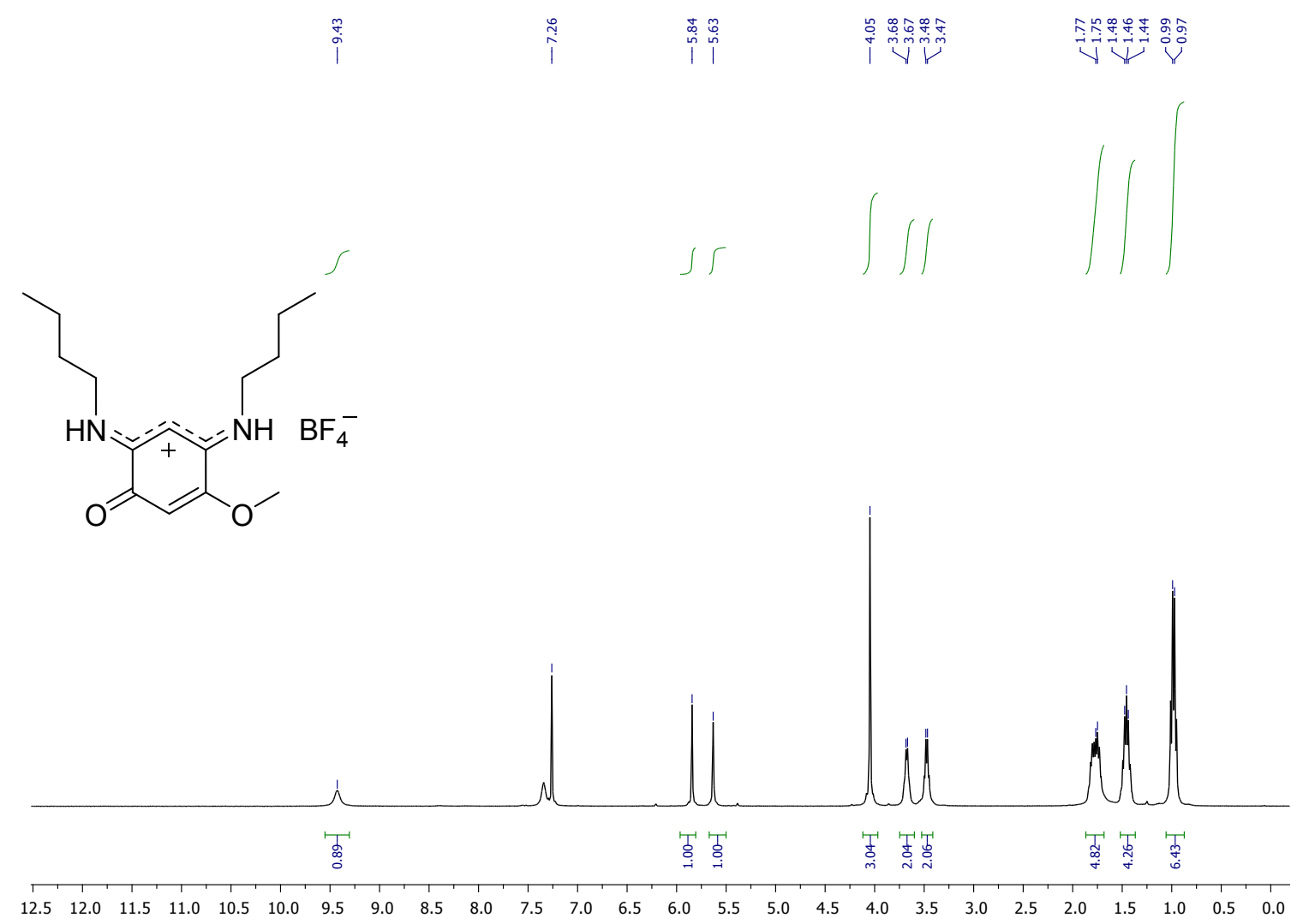

Figure S 4. ${ }^{1} \mathrm{H} \mathrm{NMR}\left(400 \mathrm{MHz}, \mathrm{CDCl}_{3}\right)$ of compound 4. 
|
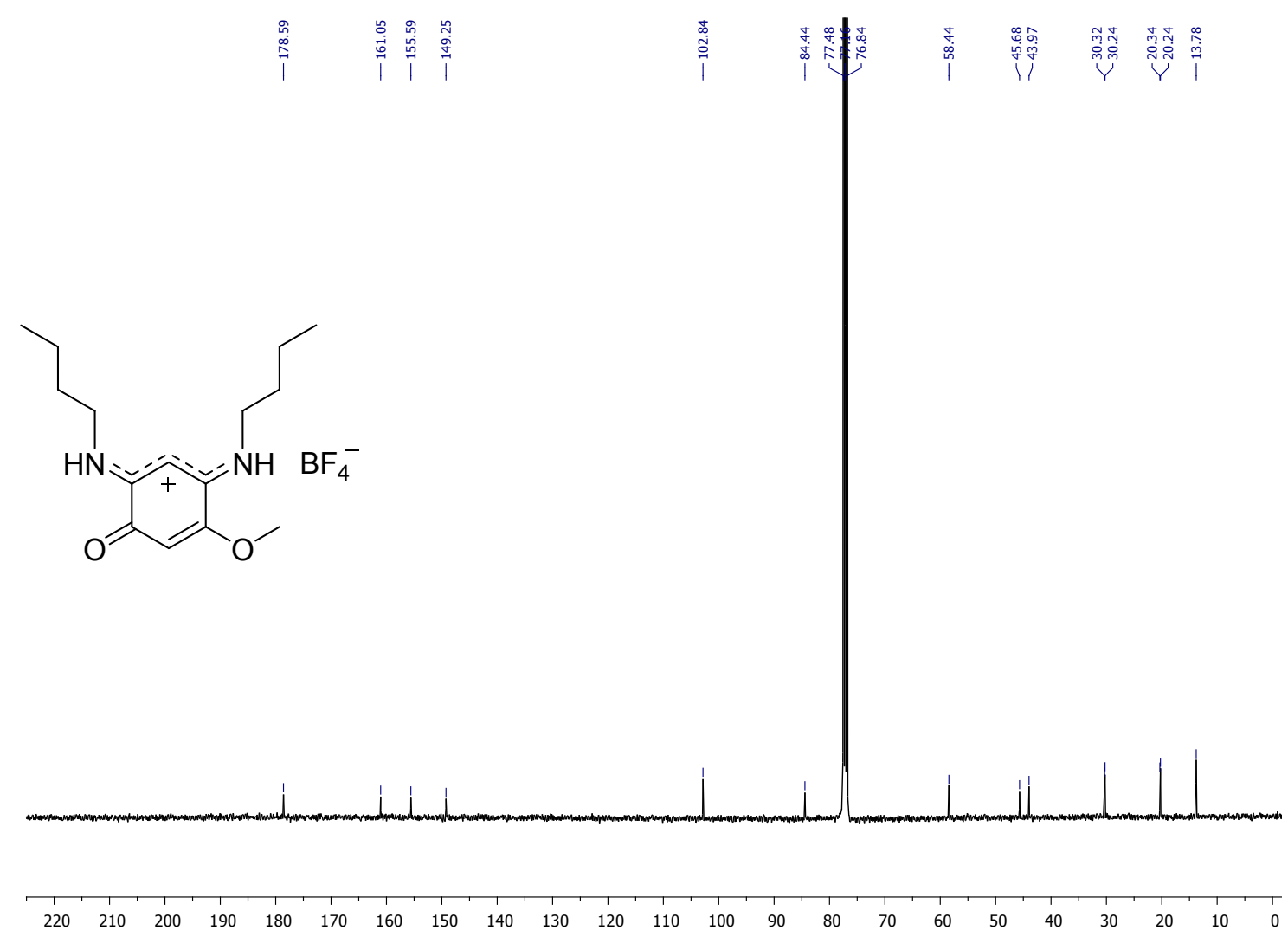

Figure S $5 .{ }^{13} \mathrm{C}$ NMR (101 MHz, $\left.\mathrm{CDCl}_{3}\right)$ of compound 4 .
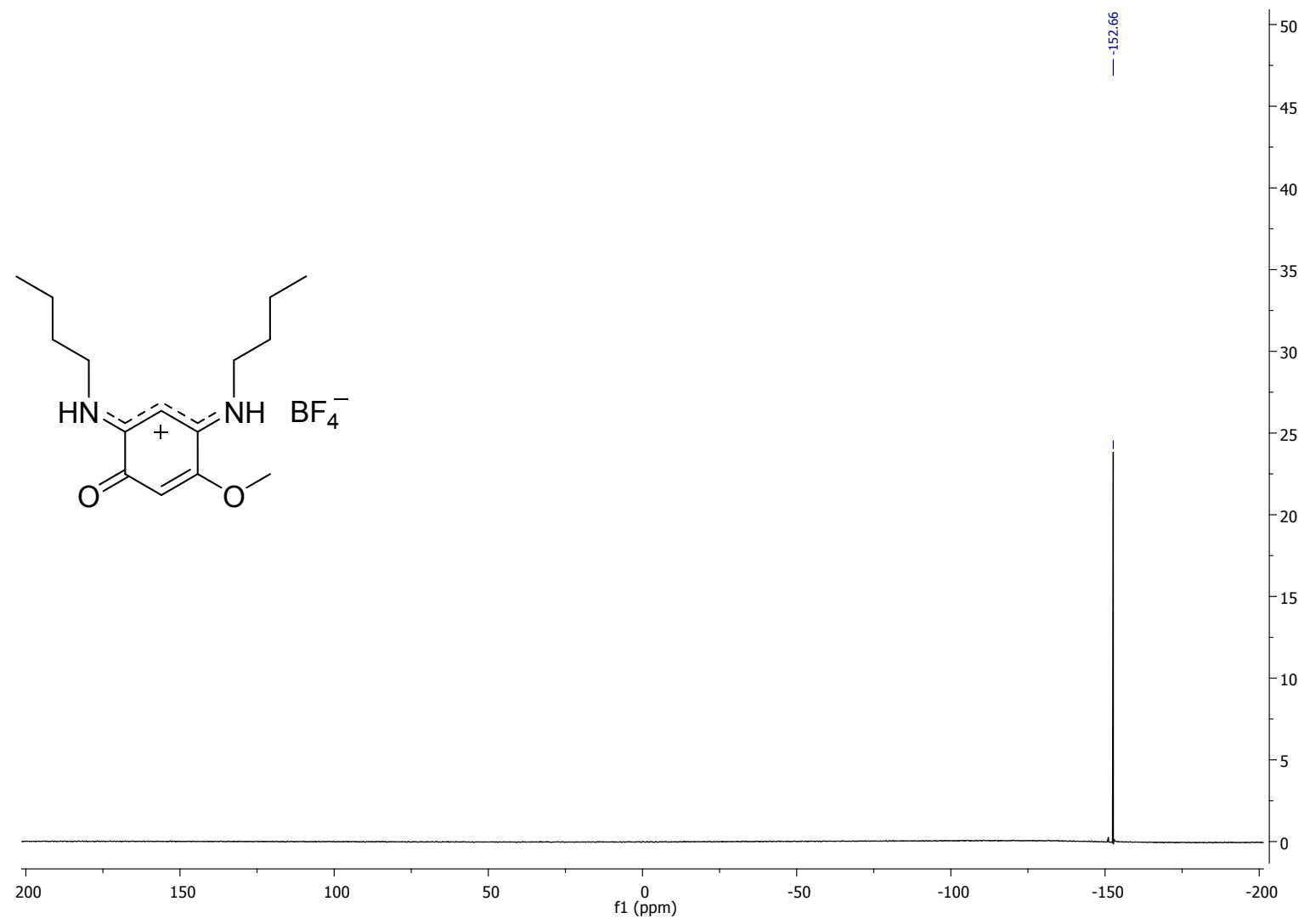

Figure S 6. ${ }^{19} \mathrm{~F}$ NMR (376 MHz, $\left.\mathrm{CDCl}_{3}\right)$ : of compound 4 .

$\mathrm{S} 10$ 


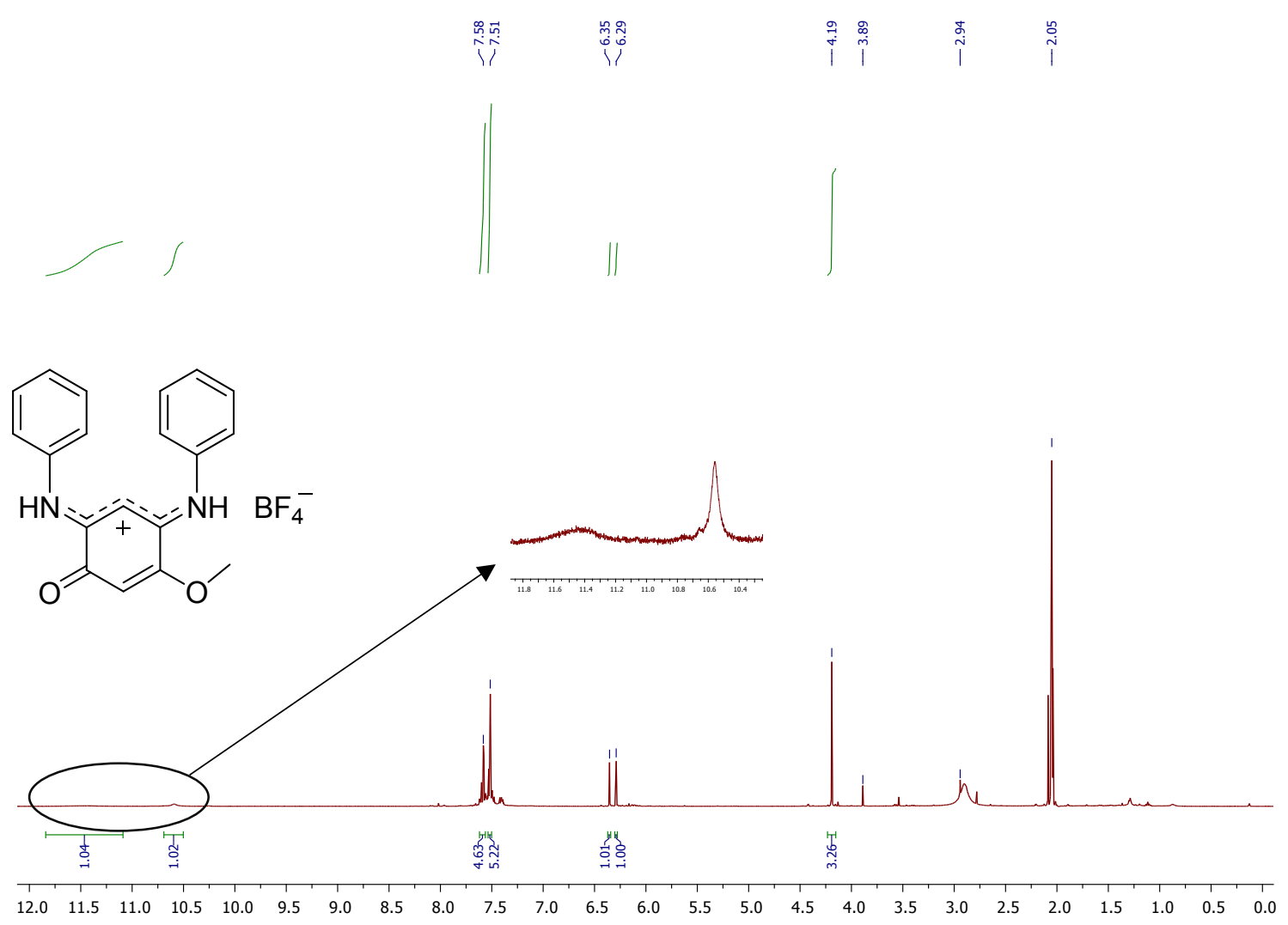

Figure S 7. ${ }^{1} \mathrm{H}$ NMR (400 MHz, Acetone- $\left.d_{6}\right)$ of compound 5a.

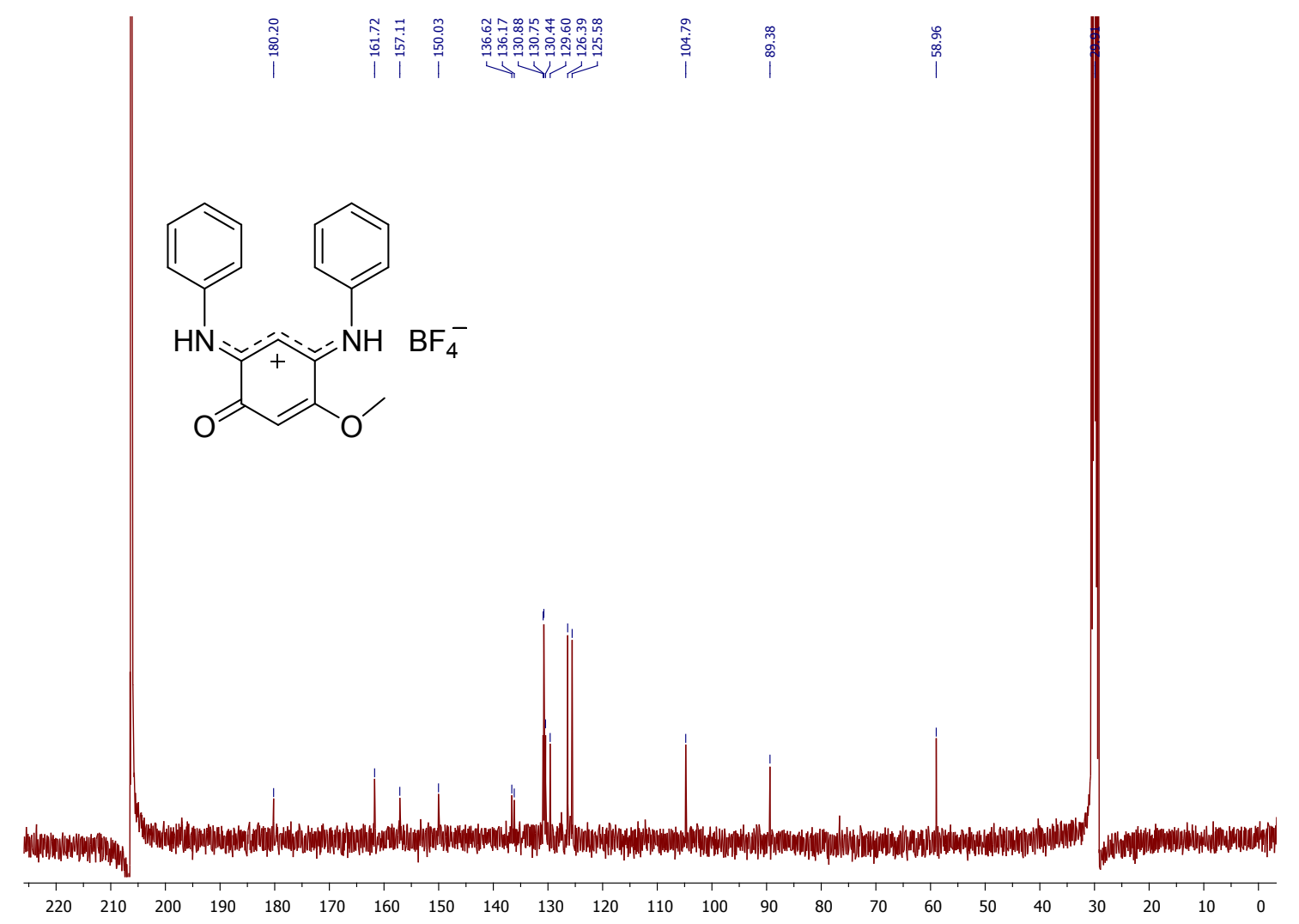

Figure S $8 .{ }^{13} \mathrm{C}$ NMR (101 MHz, Acetone- $d_{6}$ ) of compound $\mathbf{5 a}$. 
<smiles>COC1=CC(=O)C(=Nc2ccccc2)C=C1Nc1ccccc1</smiles>

Figure S 9. ${ }^{19} \mathrm{~F}$ NMR (376 MHz, Acetone-d6): of compound 5a.

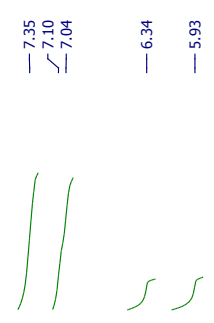<smiles>COC1=CC(=O)C(=Nc2ccc(OC)cc2)C=C1Nc1ccc(OC)cc1</smiles>

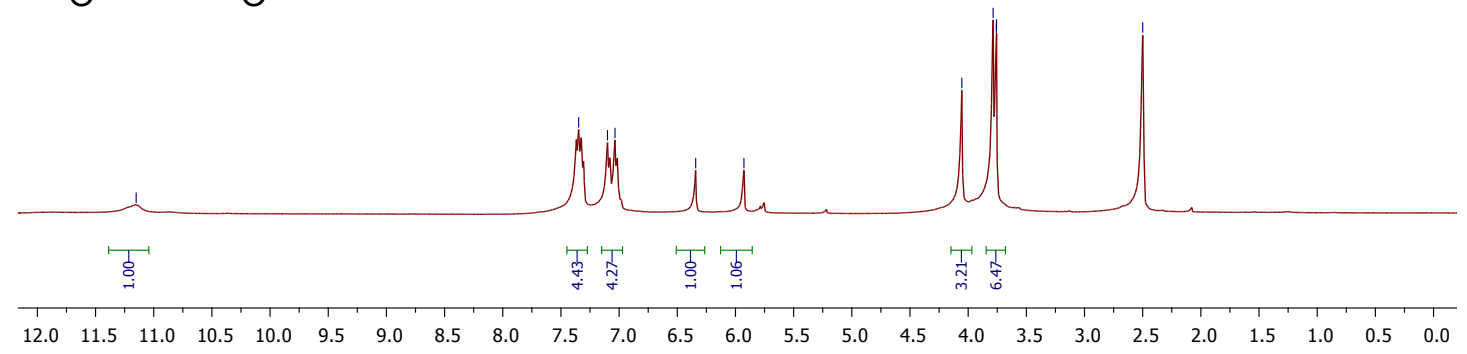

Figure $S$ 10. ${ }^{1} \mathrm{H}$ NMR $\left(400 \mathrm{MHz}, \mathrm{DMSO}-d_{6}\right)$ of compound $\mathbf{5 b}$. 
<smiles>COC1=CC(=O)C(Nc2ccc(OC)cc2)=CC1=Nc1ccc(OC)cc1</smiles>

$\begin{array}{lllllllllllllllllllllll}220 & 210 & 200 & 190 & 180 & 170 & 160 & 150 & 140 & 130 & 120 & 110 & 100 & 90 & 80 & 70 & 60 & 50 & 40 & 30 & 20 & 10 & 0\end{array}$

Figure $S 11 .{ }^{13} \mathrm{C}$ NMR (101 MHz, DMSO- $\left.d_{6}\right)$ of compound $\mathbf{5 b}$.

ATR-148-F-dmso single_pulse
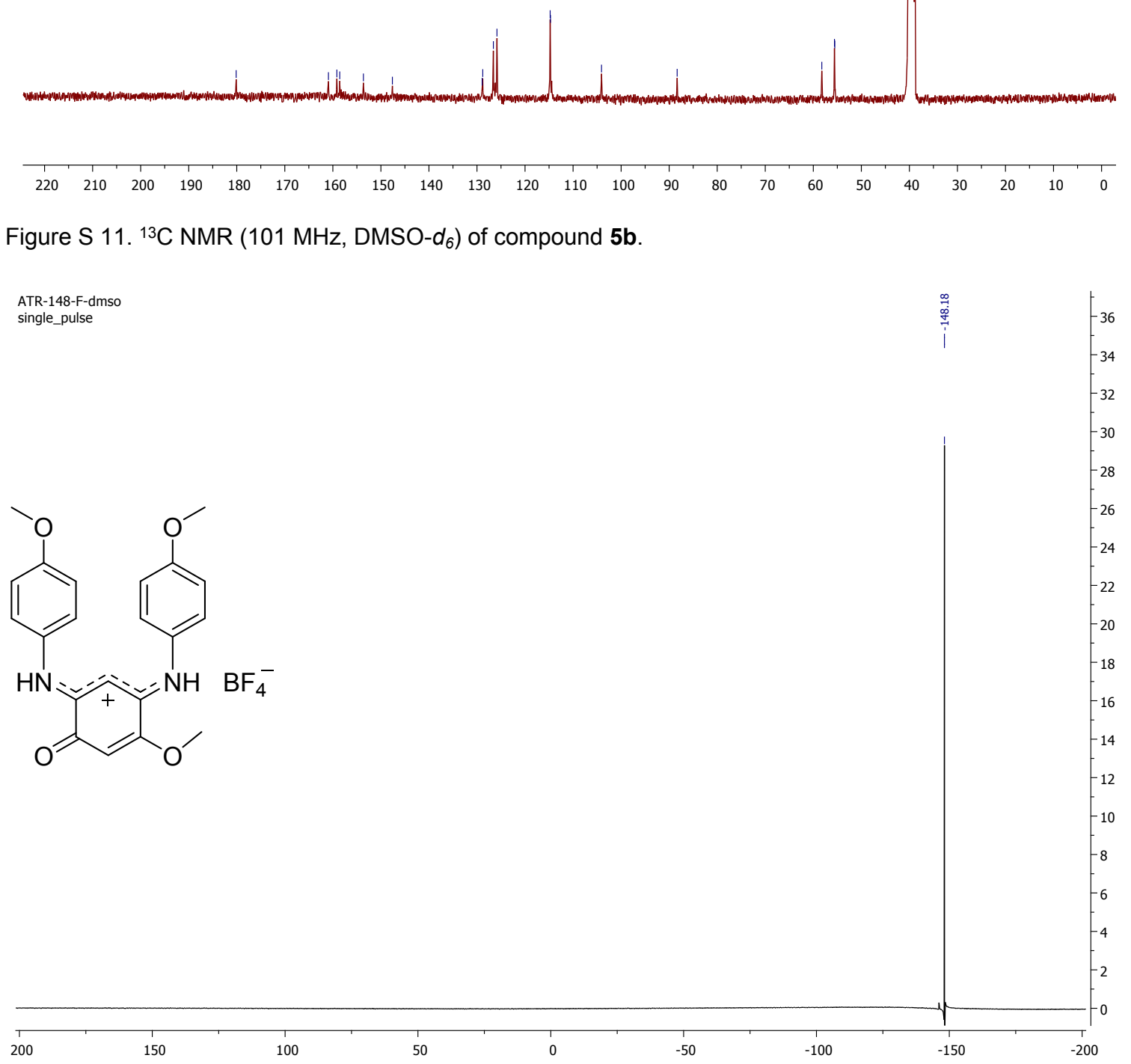

Figure S 12. ${ }^{19} \mathrm{~F}$ NMR ( $376 \mathrm{MHz}$, Acetone- $\left.d_{6}\right)$ : of compound $\mathbf{5 b}$. 


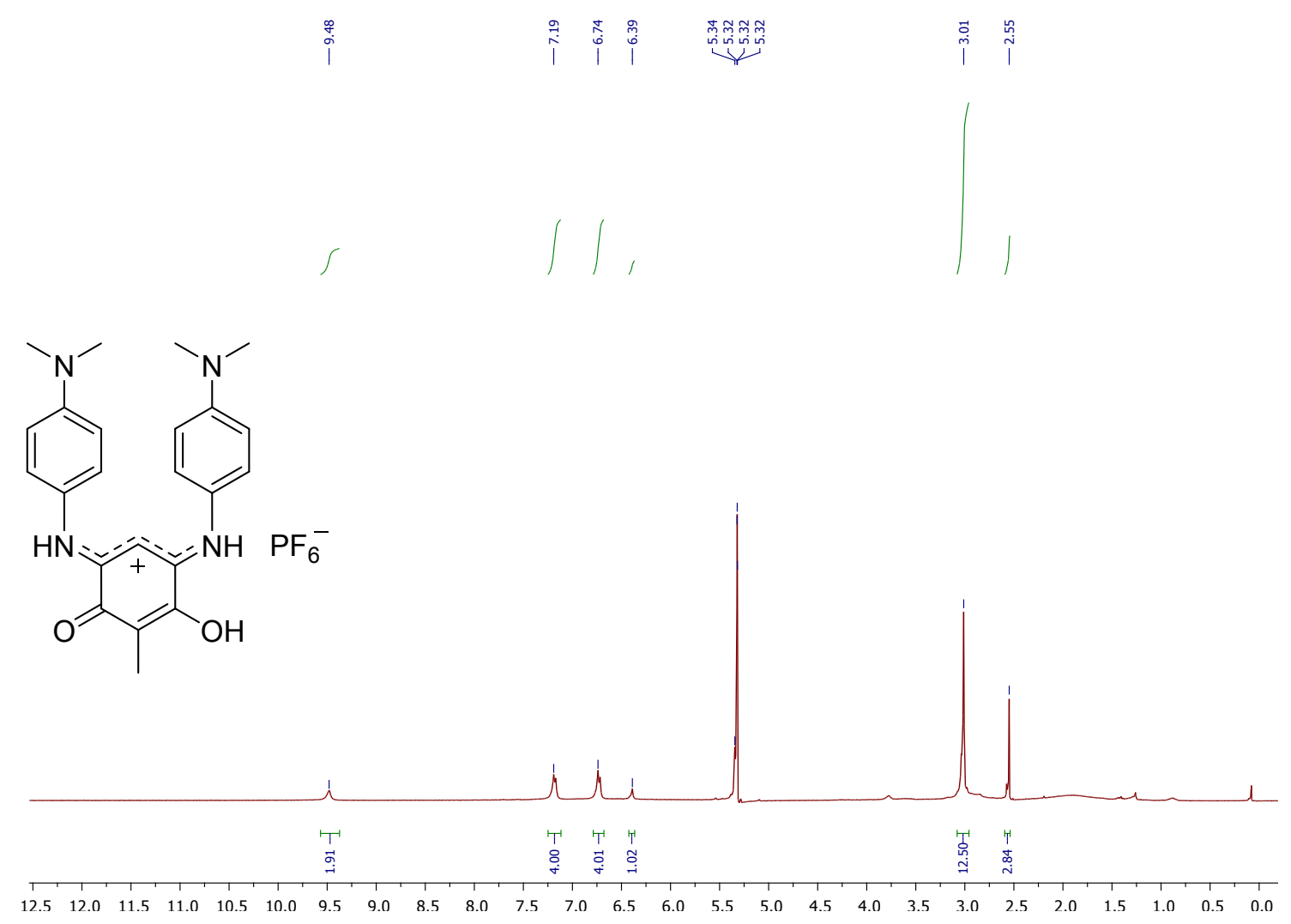

Figure $\mathrm{S} 13 .{ }^{1} \mathrm{H}$ NMR $\left(400 \mathrm{MHz}, \mathrm{CD}_{2} \mathrm{Cl}_{2}\right)$ of compound 6.
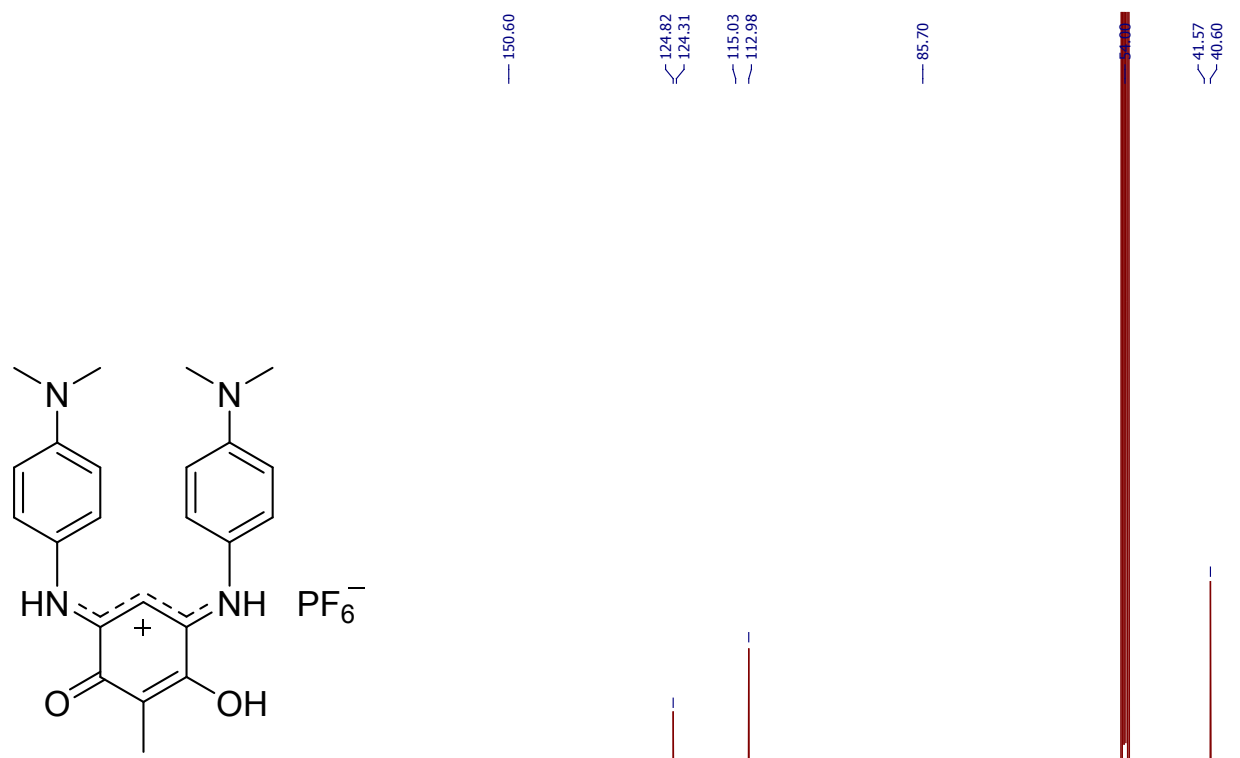

$\begin{array}{llllllllllllllllllllll}220 & 210 & 200 & 190 & 180 & 170 & 160 & 150 & 140 & 130 & 120 & 110 & 100 & 90 & 80 & 70 & 60 & 50 & 40 & 30 & 20 & 10\end{array}$

Figure $\mathrm{S} 14 .{ }^{13} \mathrm{C}$ NMR $\left(101 \mathrm{MHz}, \mathrm{CD}_{2} \mathrm{Cl}_{2}\right)$ of compound 6 . 


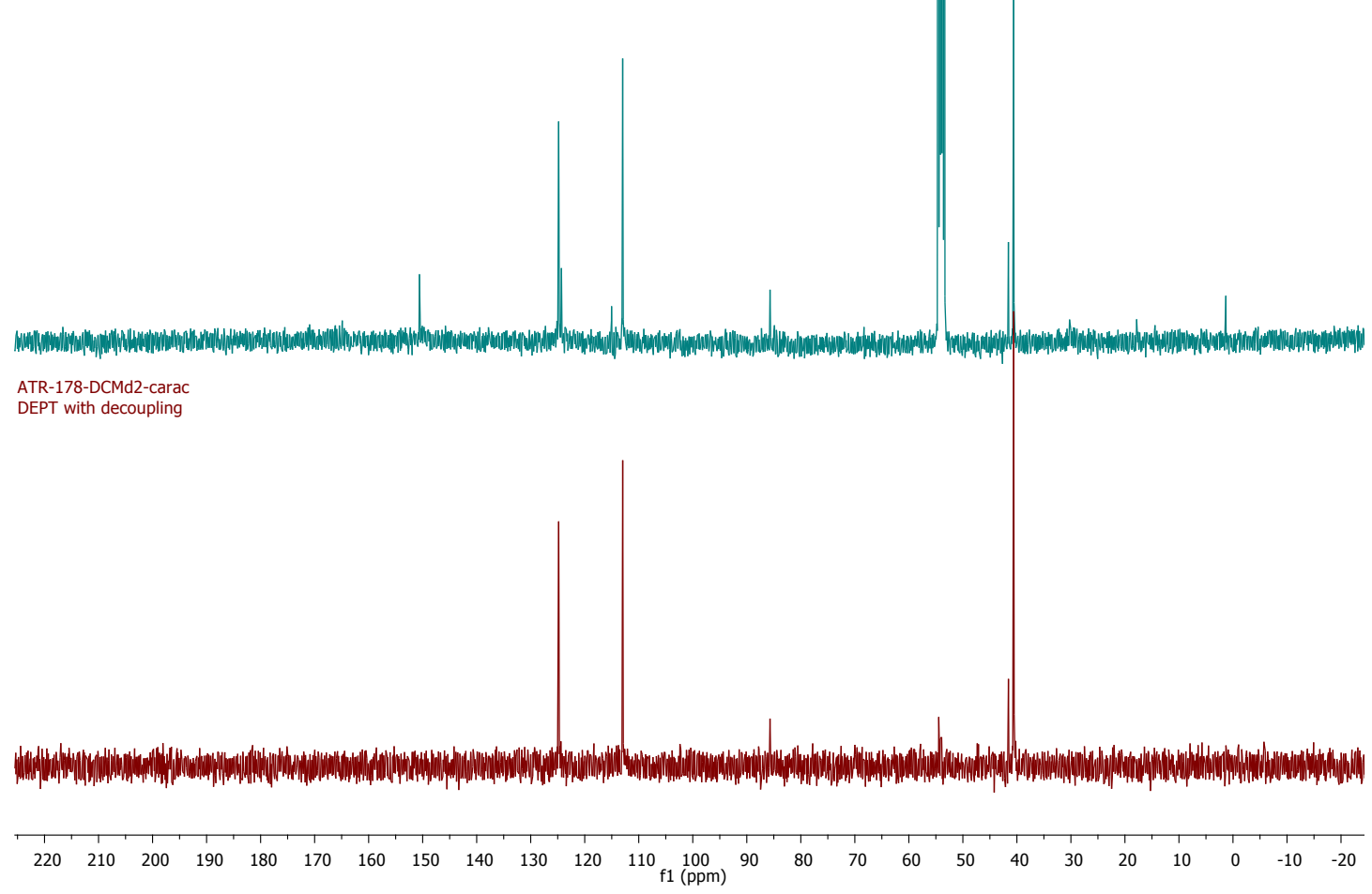

Figure $\mathrm{S}$ 15. Comparison between ${ }^{13} \mathrm{C}$ (top) and DEPT (bottom) NMR spectra (101 MHz, $\mathrm{CD}_{2} \mathrm{Cl}_{2}$ ) of compound 6 .<smiles>CC1=C(O)C(=Nc2ccc(N(C)C)cc2)C=C(Nc2ccc(N(C)C)cc2)C1=O</smiles>

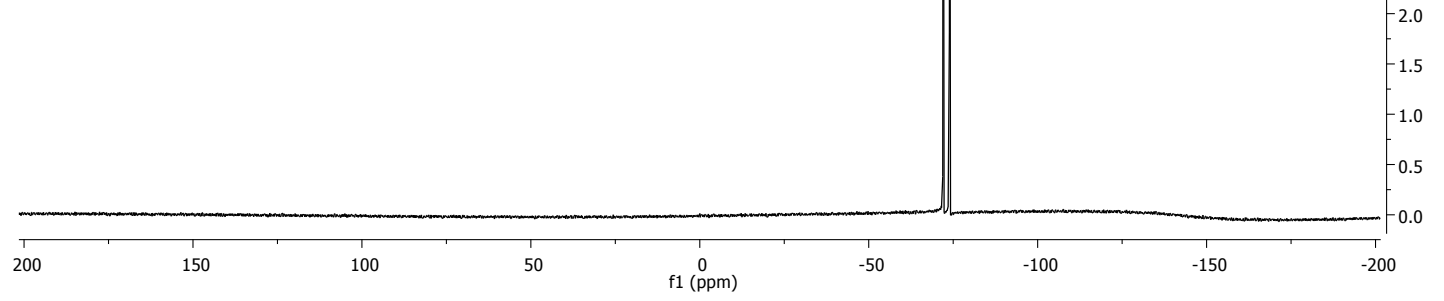

Figure S 16. ${ }^{19} \mathrm{~F}$ NMR (376 MHz, $\left.\mathrm{CD}_{2} \mathrm{Cl}_{2}\right)$ : of compound 6 . 


\section{MASS SPECTROMETRY}

ATR-012_Mex3 3 (0.086) AM2 (Ar,18000.0,0.00,0.00); Cm (1:20)

321.1344

1: TOF MS ES+ $6.42 \mathrm{e} 6$

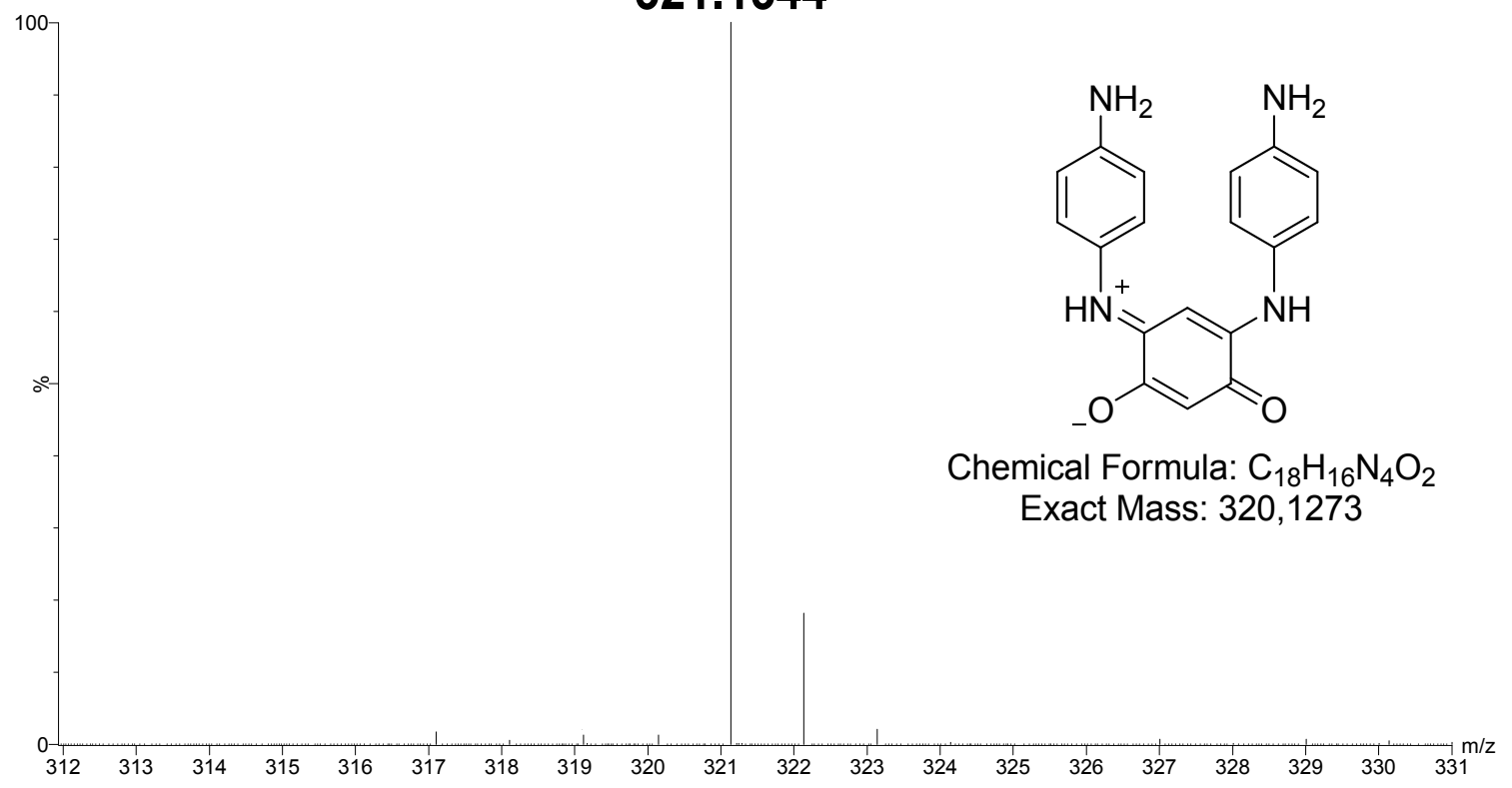

Figure S 17. HRMS spectrum of compound 3c.

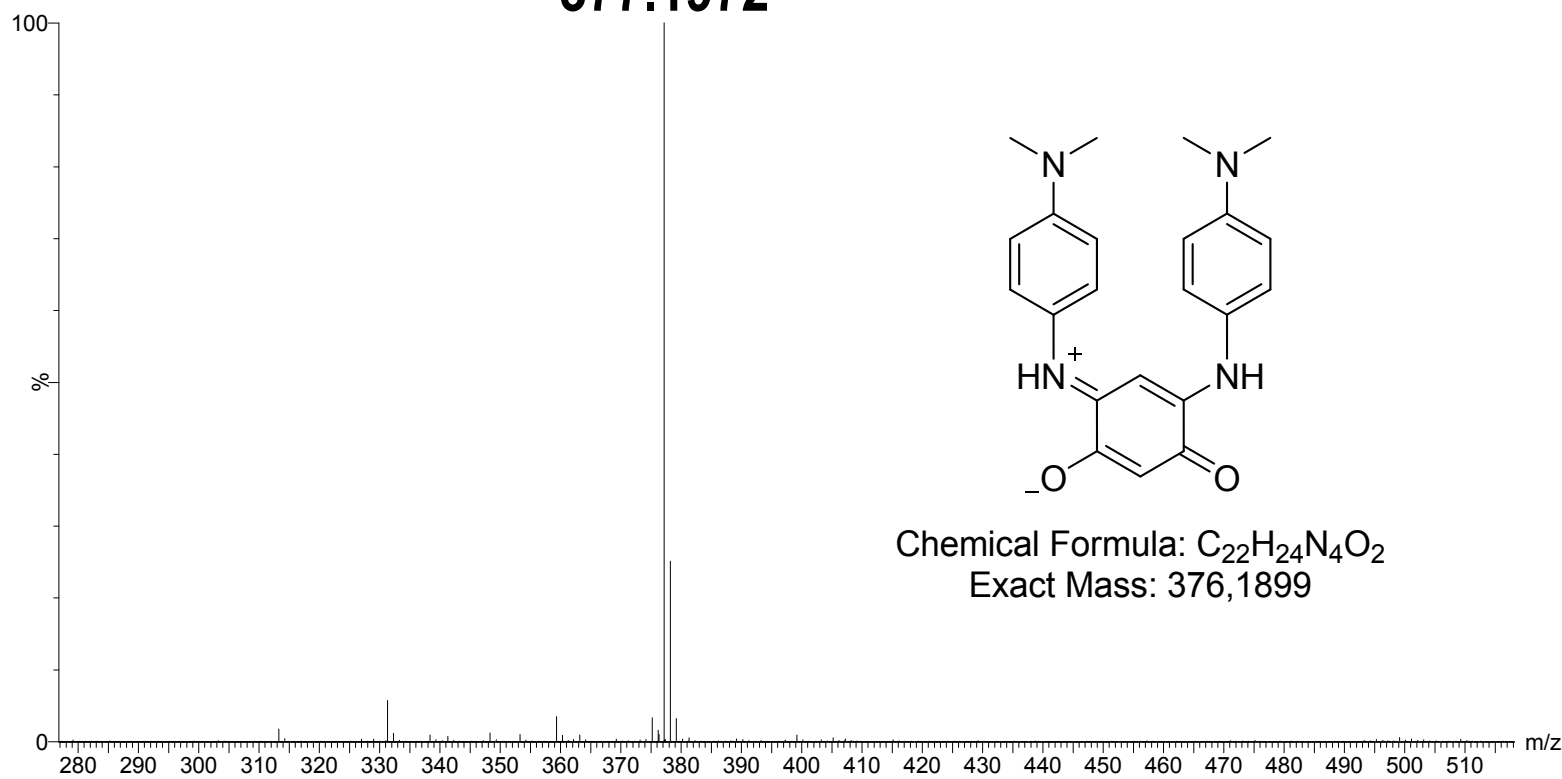

Figure S 18. HRMS spectrum of compound $\mathbf{3 d}$. 


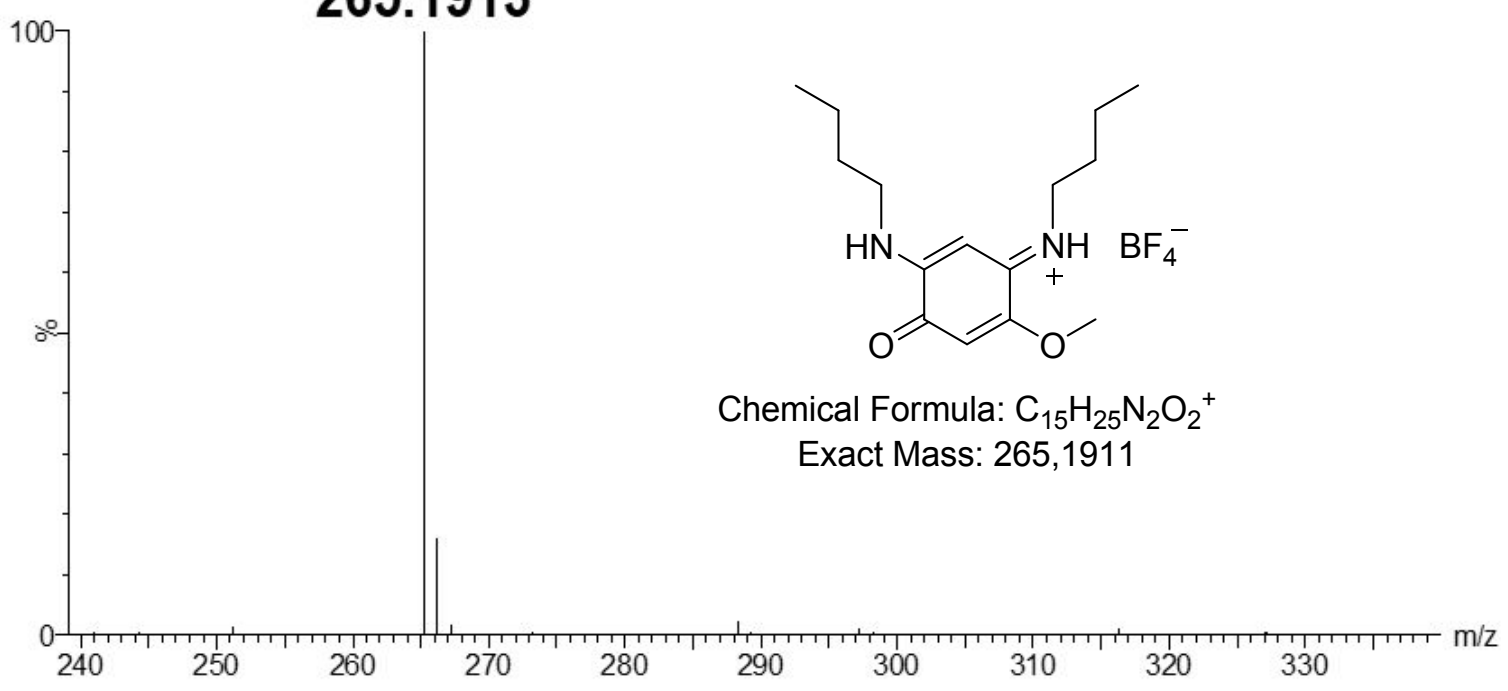

Figure S 19. HRMS spectrum of compound 4.

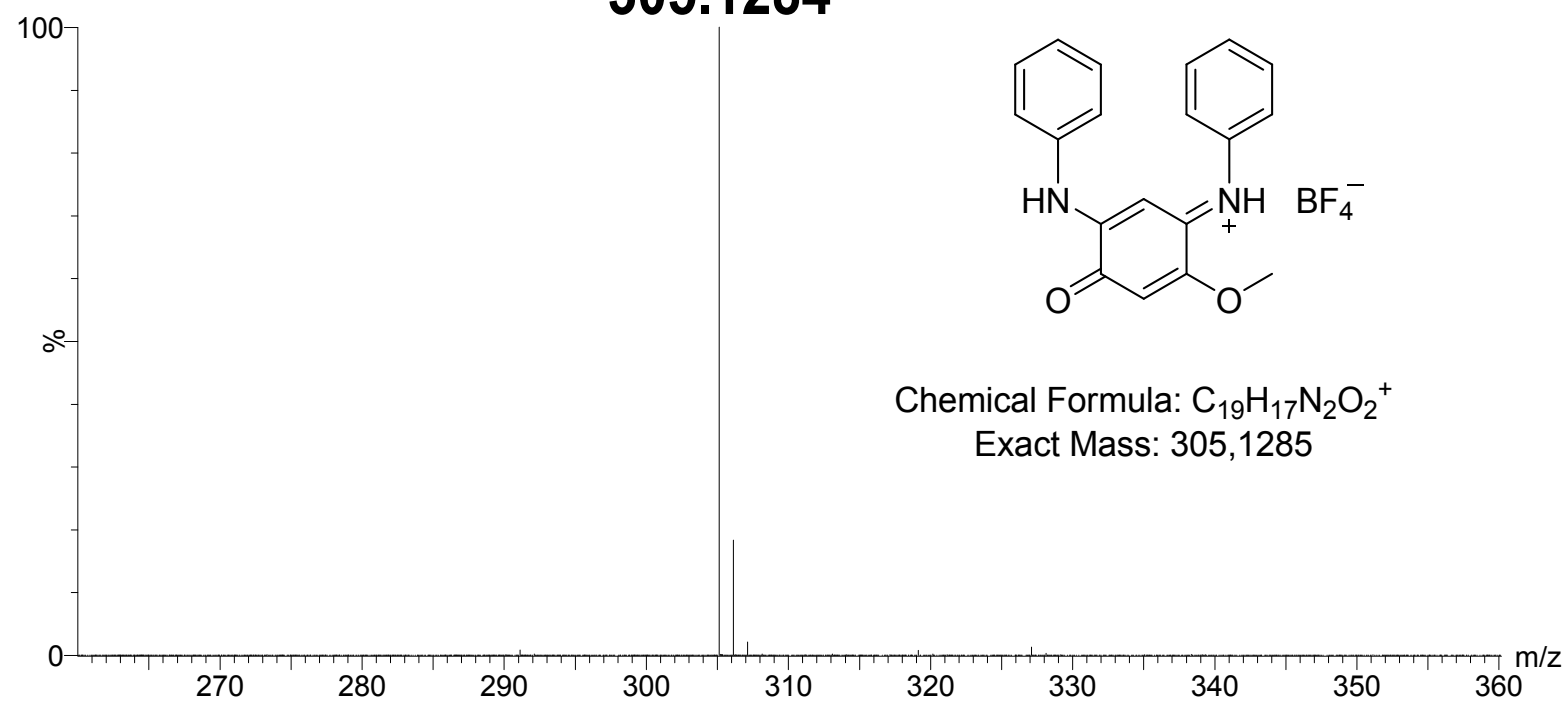

Figure S 20. HRMS spectrum of compound $\mathbf{5 a}$. 


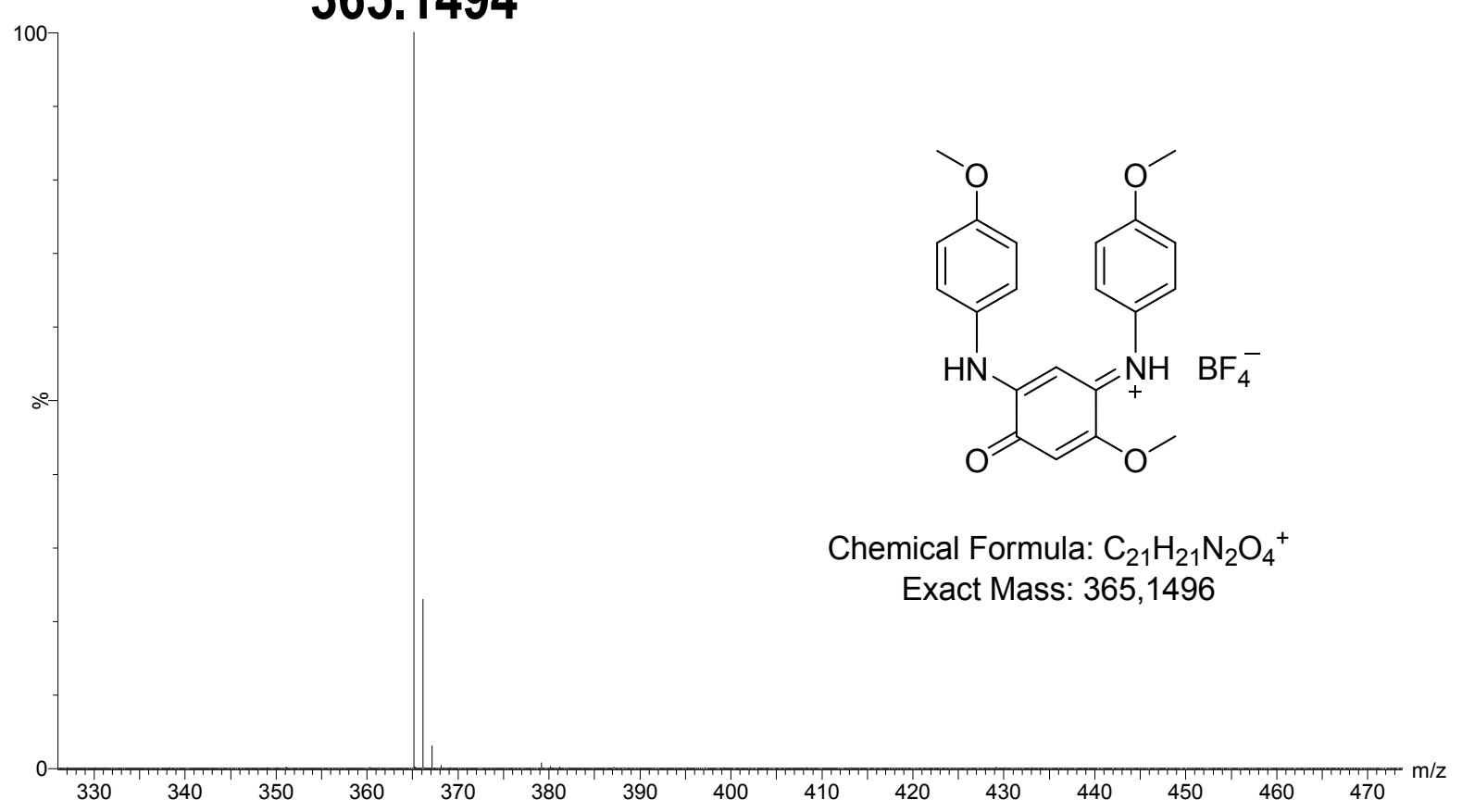

Figure S 21. HRMS spectrum of compound $\mathbf{5 b}$.

\subsection{6}

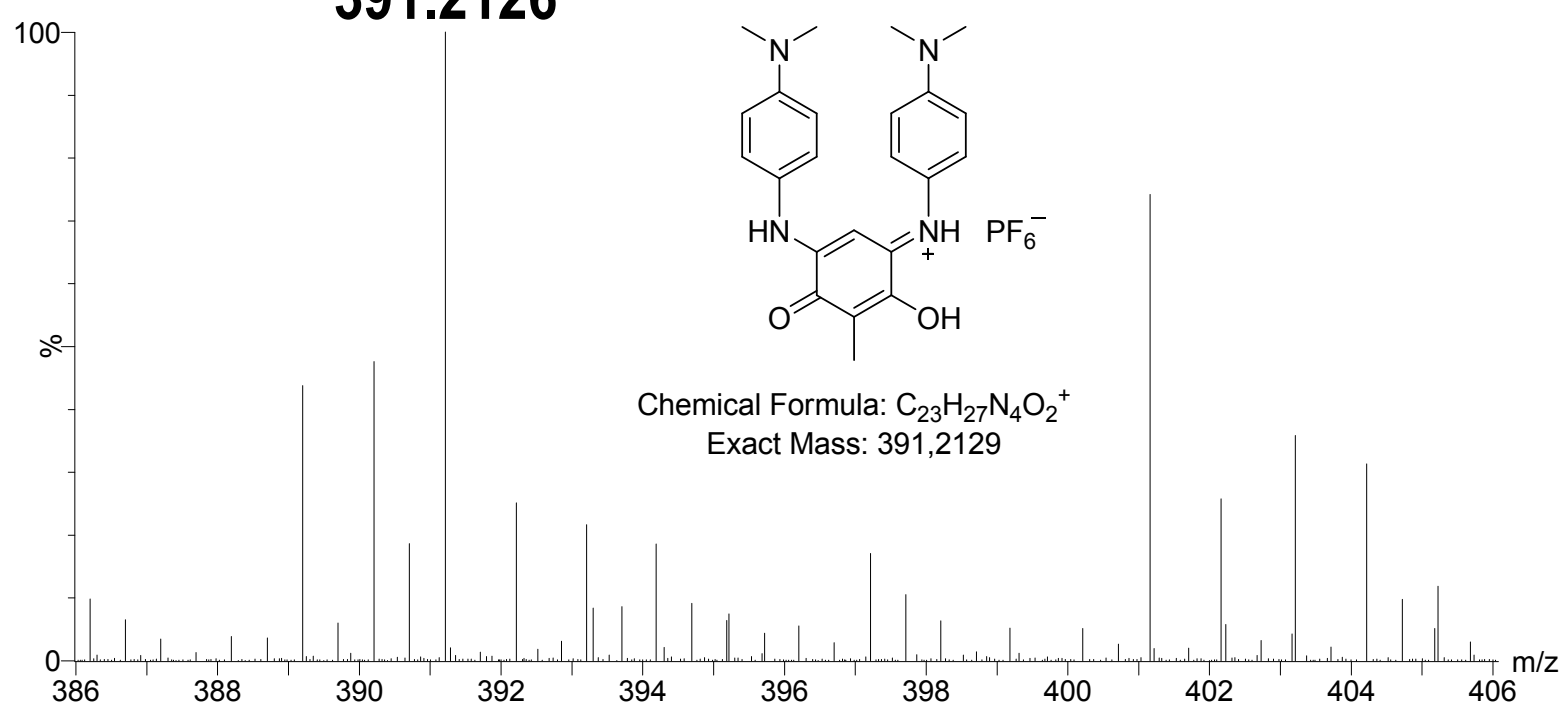

Figure S 22. HRMS spectrum of compound 6. 


\section{INFRARED SPECTROSCOPY}

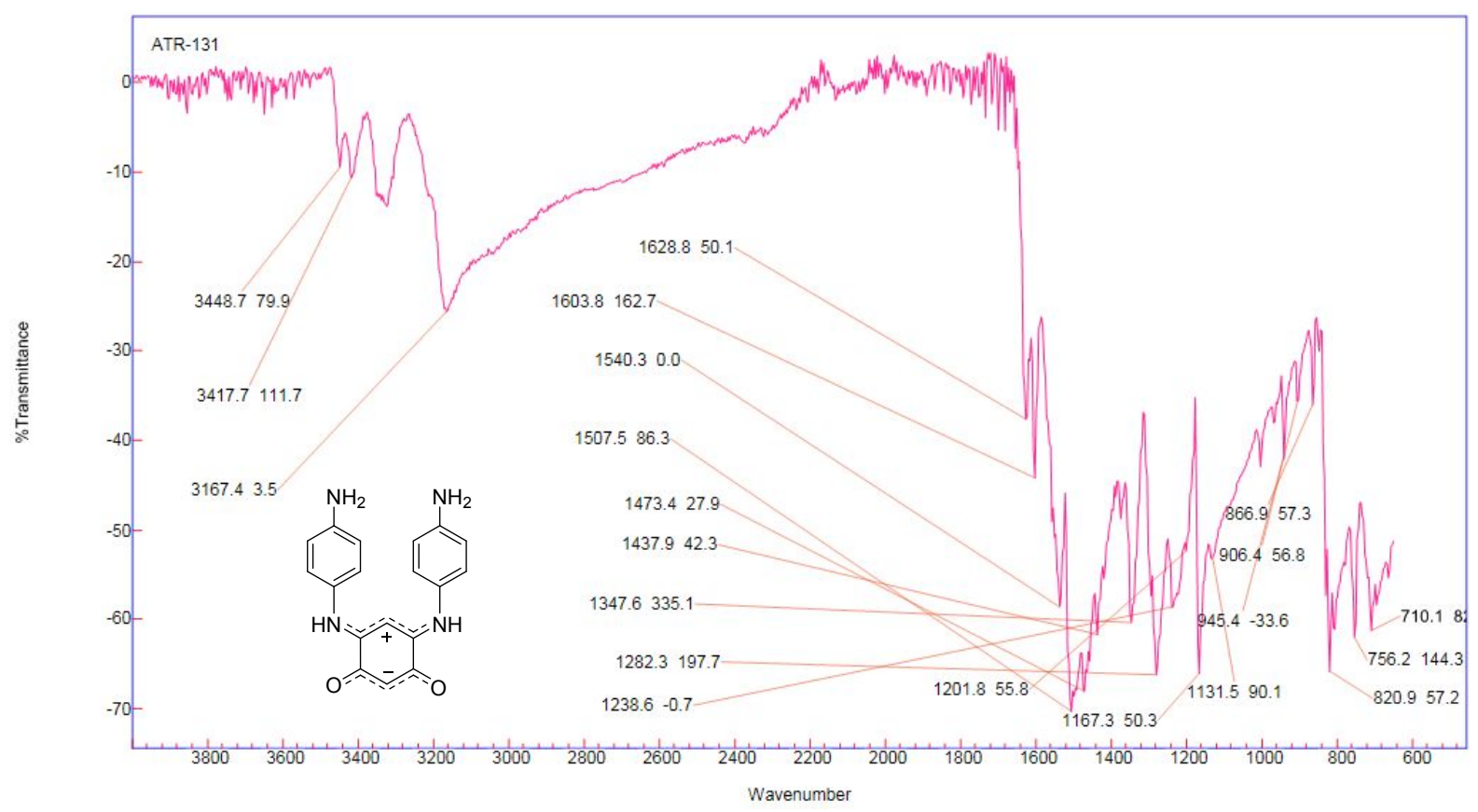

Figure S 23. Infrared spectrum of compound 3c.

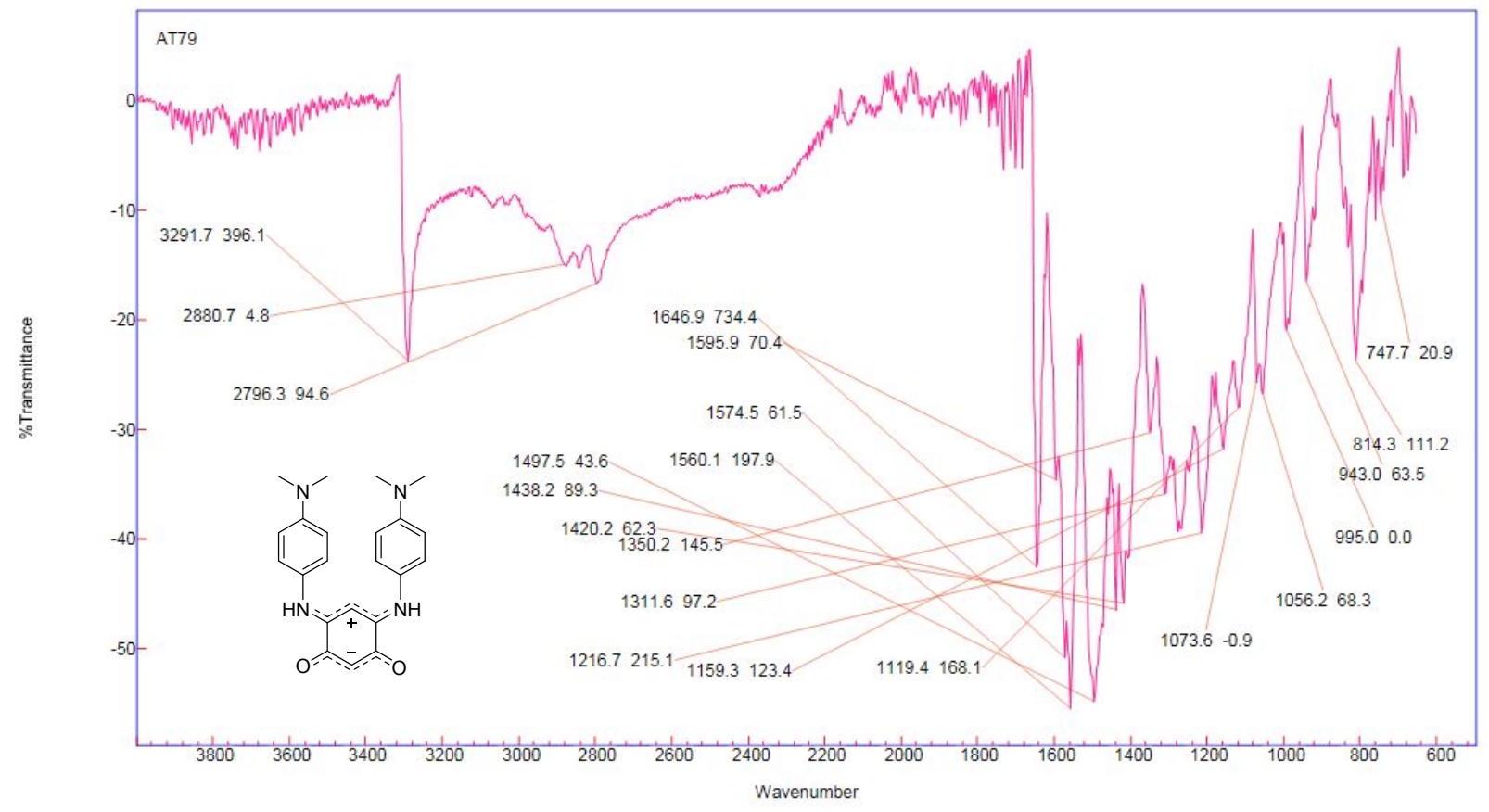

Figure S 24. Infrared spectrum of compound 3d. 


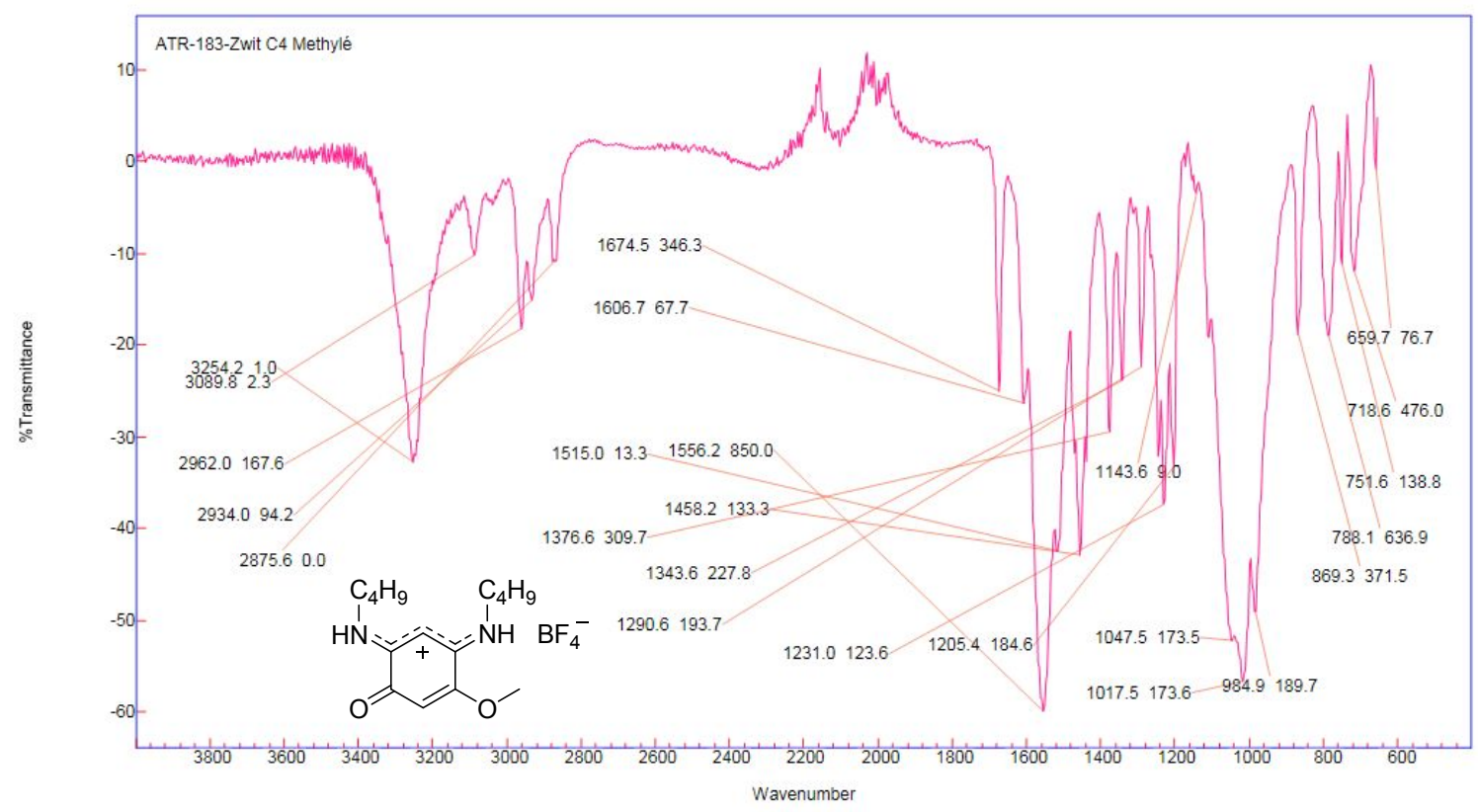

Figure S 25. Infrared spectrum of compound 4.

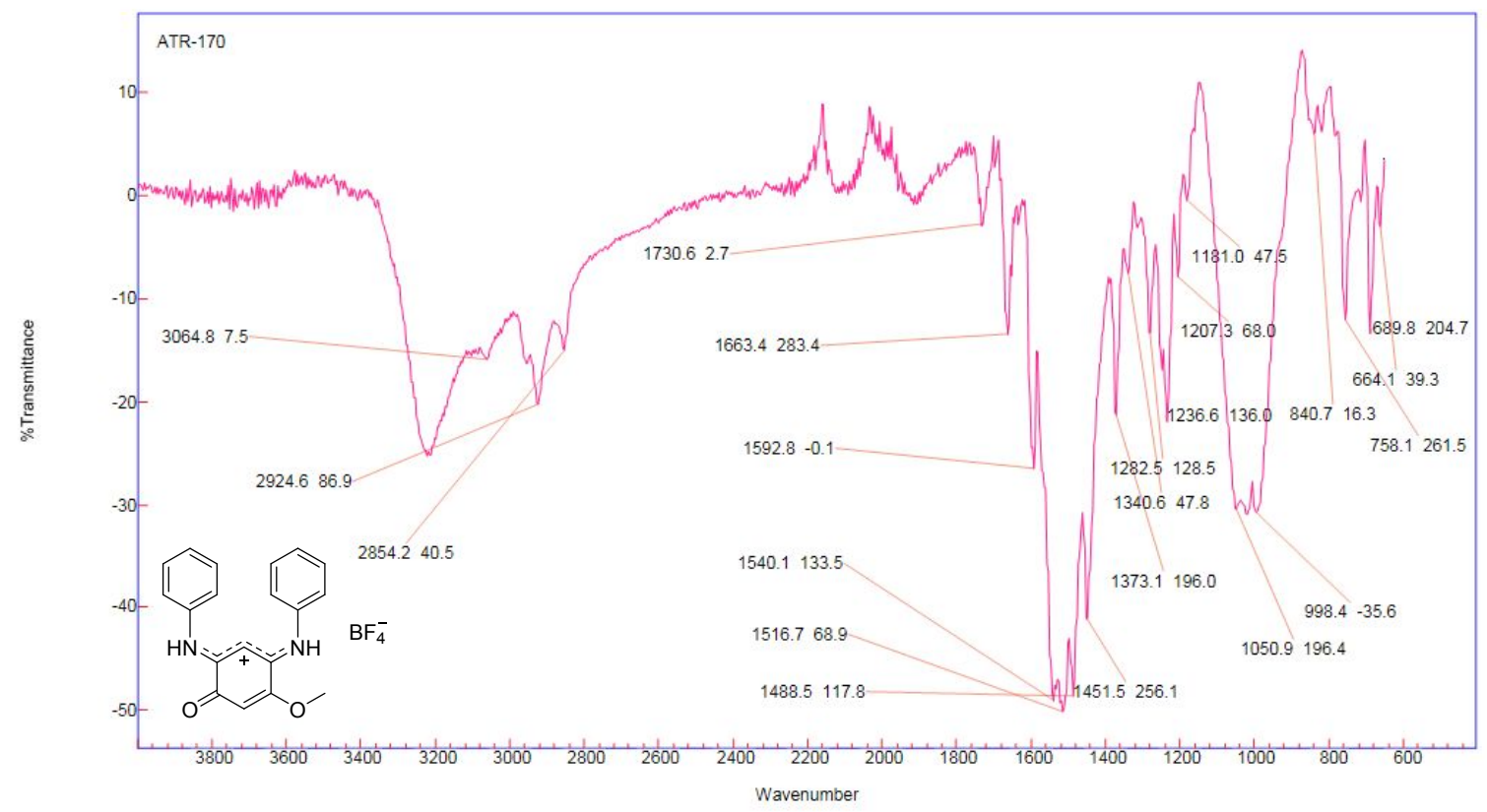

Figure S 26. Infrared spectrum of compound $\mathbf{5 a}$. 


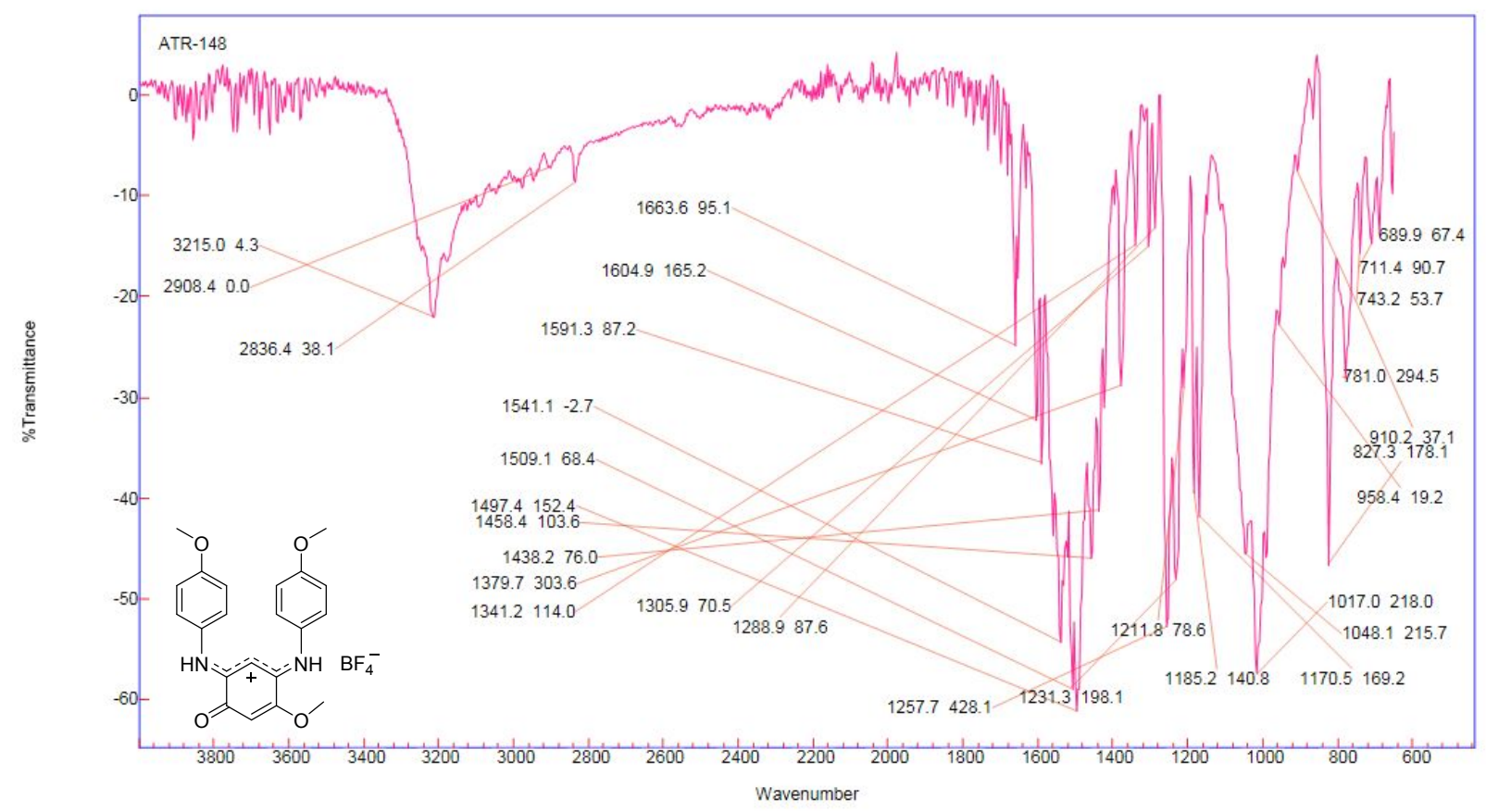

Figure S 27. Infrared spectrum of compound $\mathbf{5 b}$.

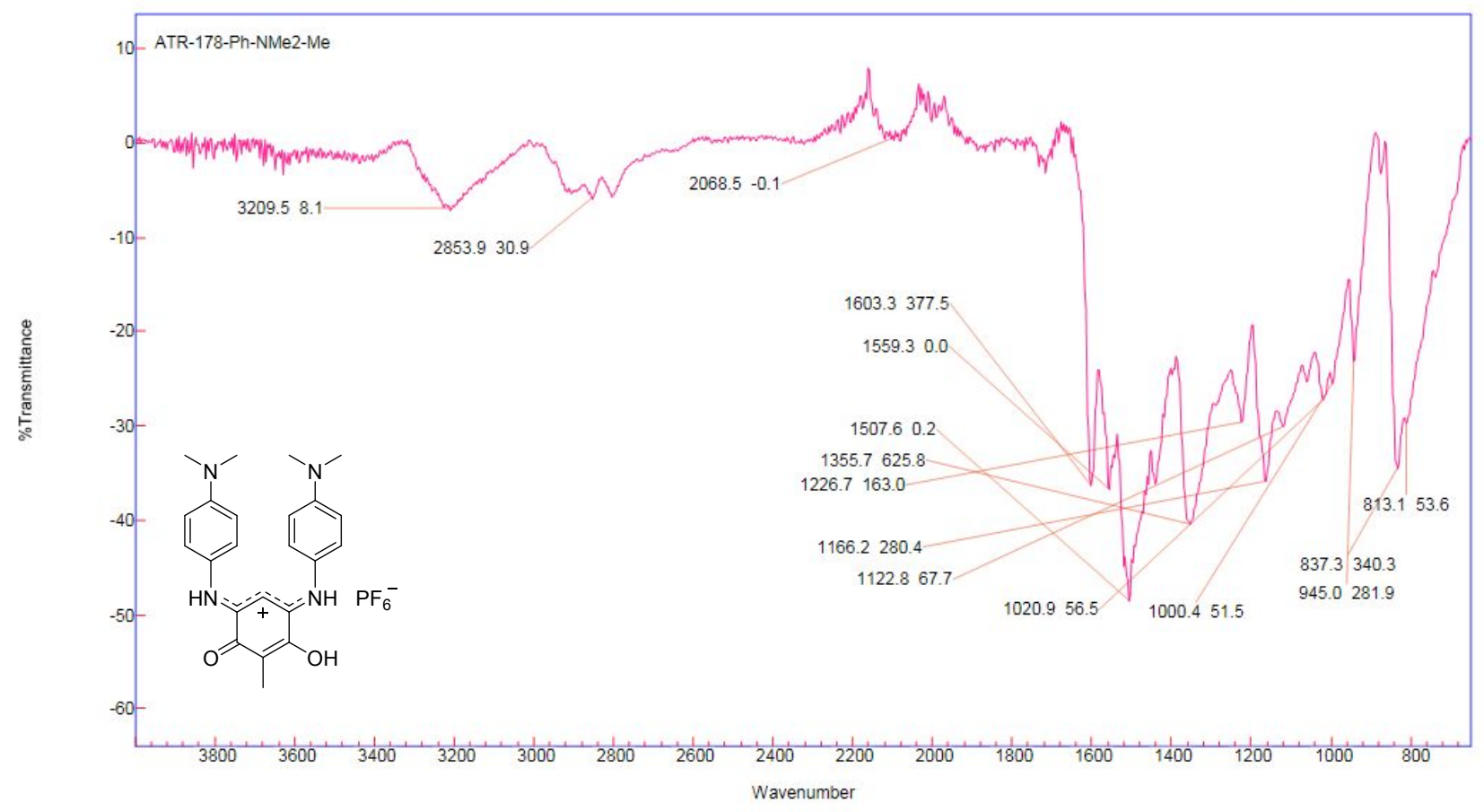

Figure S 28. Infrared spectrum of compound $\mathbf{6}$. 


\section{X-RAY DIFFRACTION}

A single crystal suitable for diffraction analysis was obtained by slow diffusion of an acetone solution containing the dye 2 towards pentane. The single crystal structure shows that the compound crystallizes as dimers arranged in a head-to-tail fashion via hydrogen bonding interactions (Figure $S$ 29). The bond length alternation measured in the cationic and anionic subunits (BLA $=0$ and $0.002 \AA$, respectively), together with the length of the bond binding them $(1.52 \AA)$ indubitably confirms the zwitterionic nature of the solid state structure.

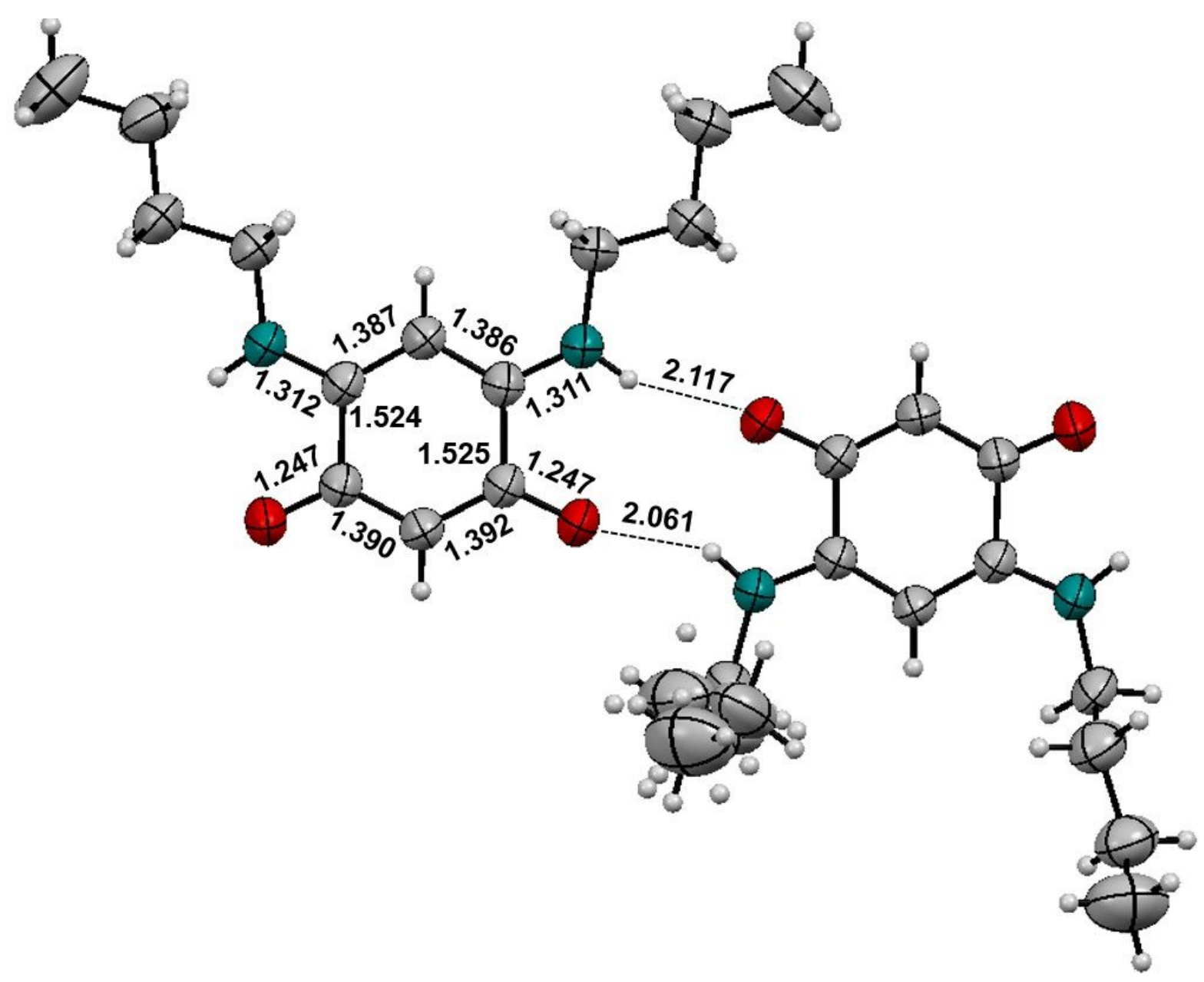

Figure S 29. Single crystal structures of compound 2 with selected bond lengths $(\AA)$. Anisotropic displacement ellipsoids plot at the 50 percent probability level. Butyl chains are omitted for clarity. 
Table S 1. Crystal data and structures refinements of compound $2 \mathbf{a}$.

\begin{tabular}{|c|c|}
\hline Empirical formula & $\mathrm{C}_{28} \mathrm{H}_{44} \mathrm{~F}_{2} \mathrm{~N}_{4} \mathrm{O}_{4}$ \\
\hline $\mathrm{M}_{\mathrm{w}}$ & 500.67 \\
\hline Crystal system & triclinic \\
\hline Measurement temperature/ $\mathrm{K}$ & 295 \\
\hline Space group & $P-1$ \\
\hline $\mathrm{a} / \AA$ & $10.0649(3)$ \\
\hline $\mathrm{b} / \AA$ & $10.8776(4)$ \\
\hline $\mathrm{c} / \AA$ & $13.9305(4)$ \\
\hline$\alpha /^{\circ}$ & $99.540(3)$ \\
\hline$\beta /{ }^{\circ}$ & $103.023(2)$ \\
\hline$\gamma /^{\circ}$ & $90.287(3)$ \\
\hline $\mathrm{V} / \AA^{3}$ & $1463.94(8)$ \\
\hline z & 2 \\
\hline $\mathrm{Dc} / \mathrm{g} \cdot \mathrm{cm}^{-3}$ & 1.136 \\
\hline Crystal colour & colorless \\
\hline Crystal size/mm³ & $0.3 \times 0.16 \times 0.1$ \\
\hline$\mu(\mathrm{Mo}-\mathrm{K} \alpha) / \mathrm{mm}^{-1}$ & 0.608 \\
\hline $\mathrm{N}^{\circ}$ of refl. measured & 19154 \\
\hline $\mathrm{N}^{\circ}$ of unique refl. & 5372 \\
\hline $\mathrm{N}^{\circ}$ of observed refl. $\left[\mathrm{F}^{2}>4 \sigma \mathrm{F}^{2}\right]$ & 5670 \\
\hline $\mathrm{N}^{\circ}$ parameters refined & 347 \\
\hline $\mathrm{R}_{1}\left[\mathrm{~F}^{2}>4 \sigma \mathrm{F}^{2}\right]$ & 0.0500 \\
\hline$w R_{1}\left[F^{2}>4 \sigma F^{2}\right]$ & 0.1564 \\
\hline $\mathrm{R}_{2}$ [all refl.] & 0.0577 \\
\hline$w_{2}$ [all refl.] & 0.1665 \\
\hline Goodness of fit [all refl.] & 1.069 \\
\hline Residual Fourier/e. $\AA^{-3}$ & $-0.16,0.27$ \\
\hline
\end{tabular}


VII. CYCLIC VOLTAMMETRY
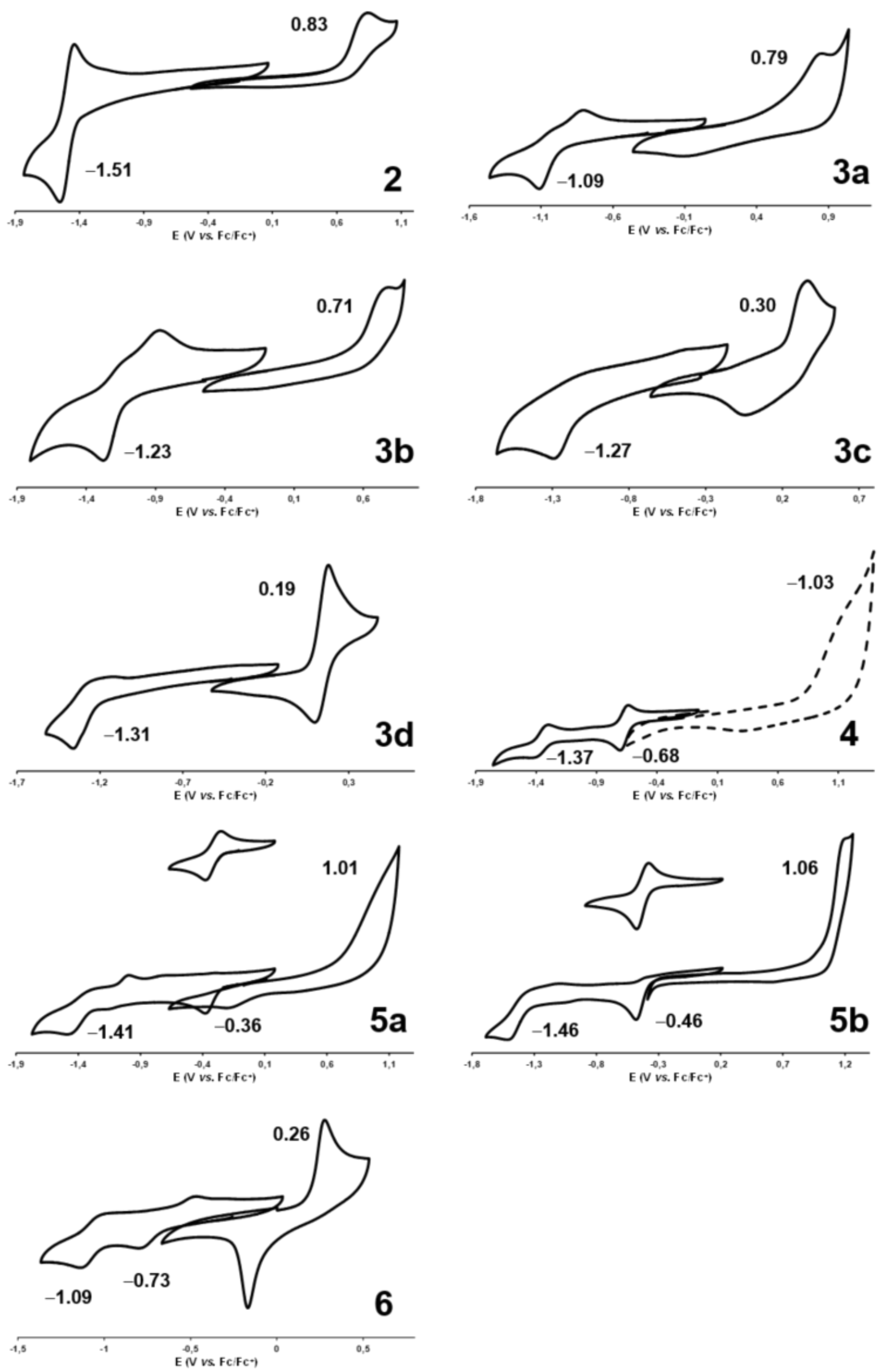

Figure S 30. Cyclic voltammograms of the compounds in DCM. 
VIII. ADDITIONAL OPTICAL SPECTRA

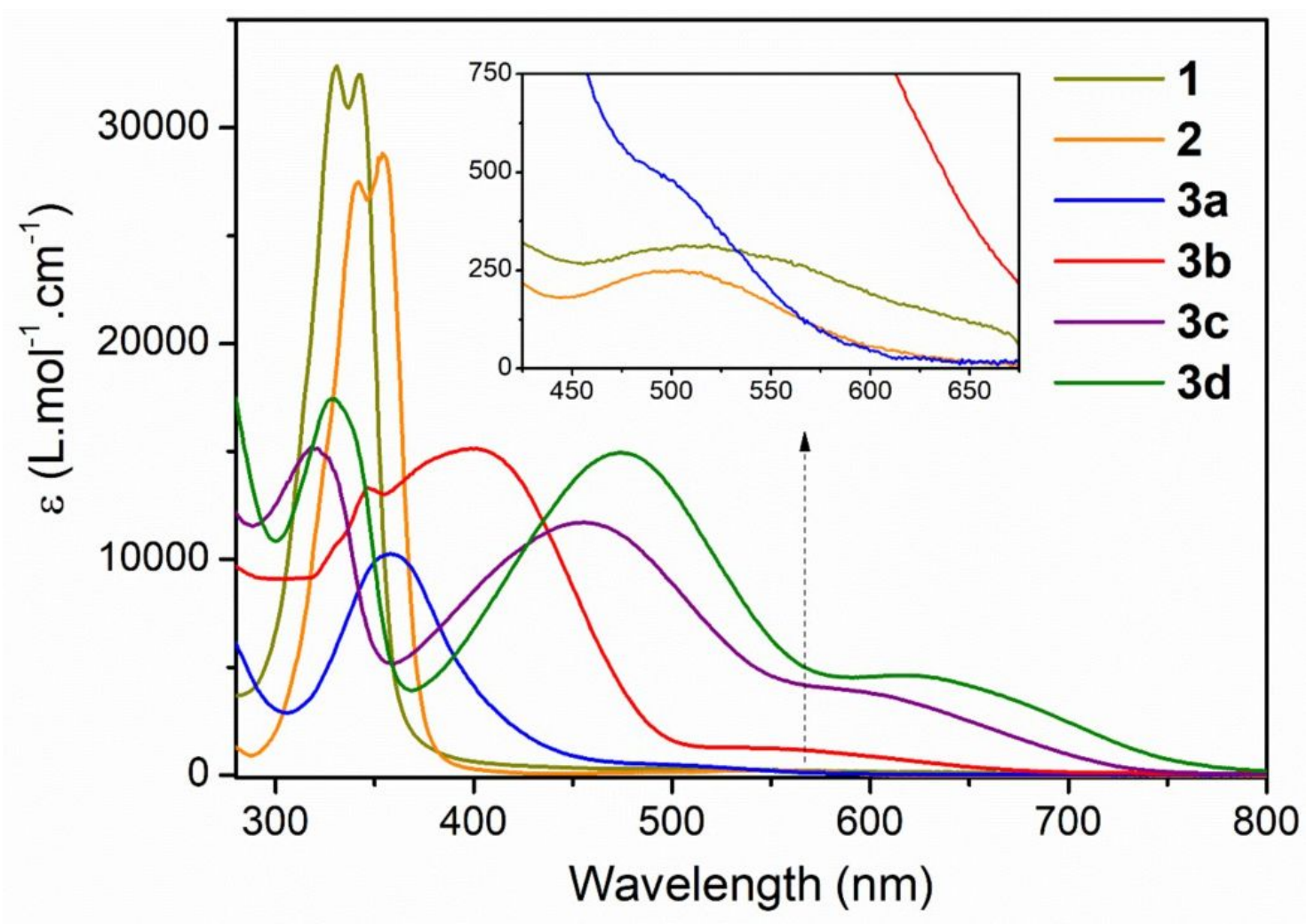

Figure S 31. Electronic absorption spectra of compounds 1-3 in DMSO solution. 

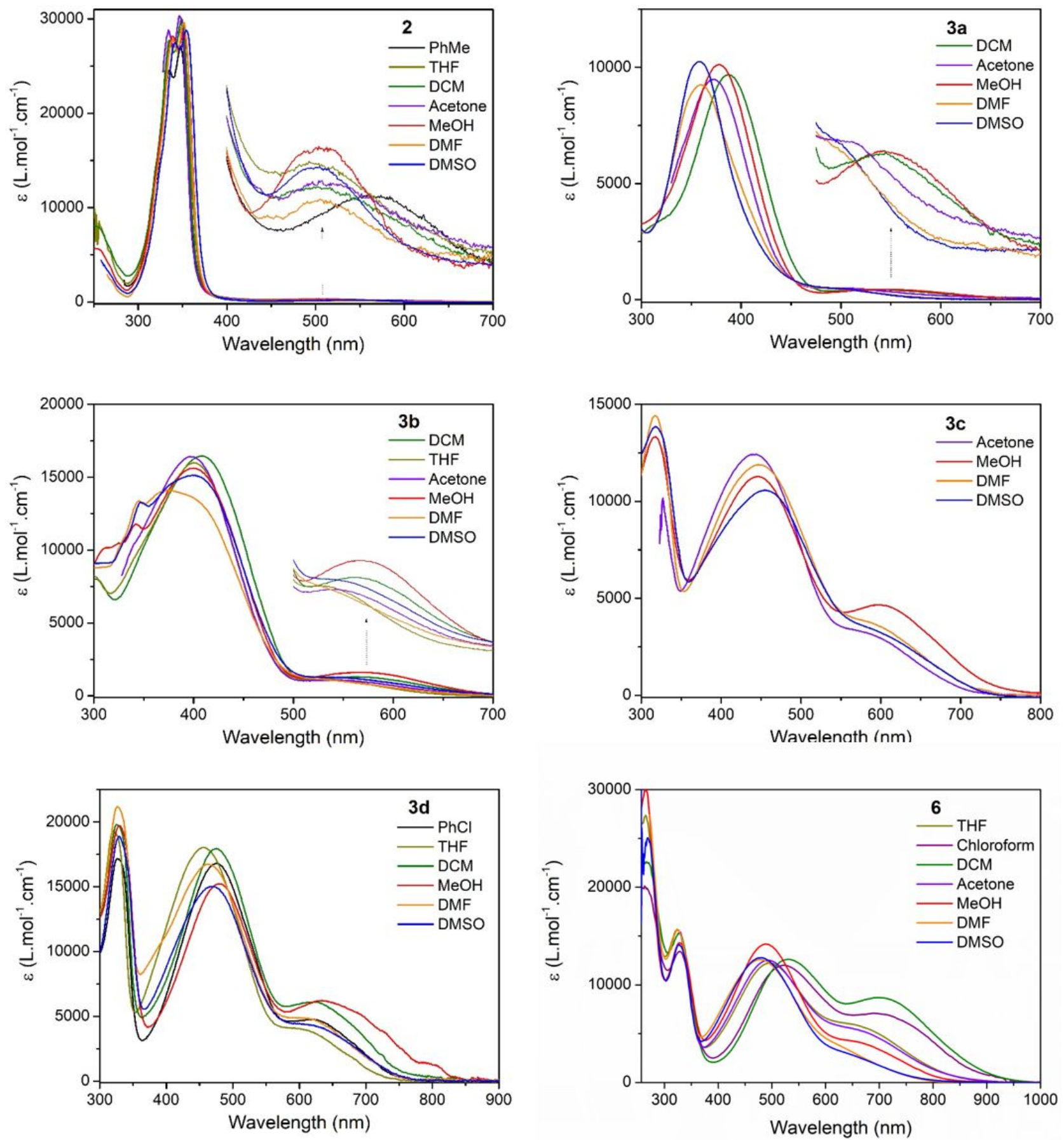

Figure S 32. Absorption solvatochromism of compounds $\mathbf{2}$, 3a-d and $\mathbf{6}$. 

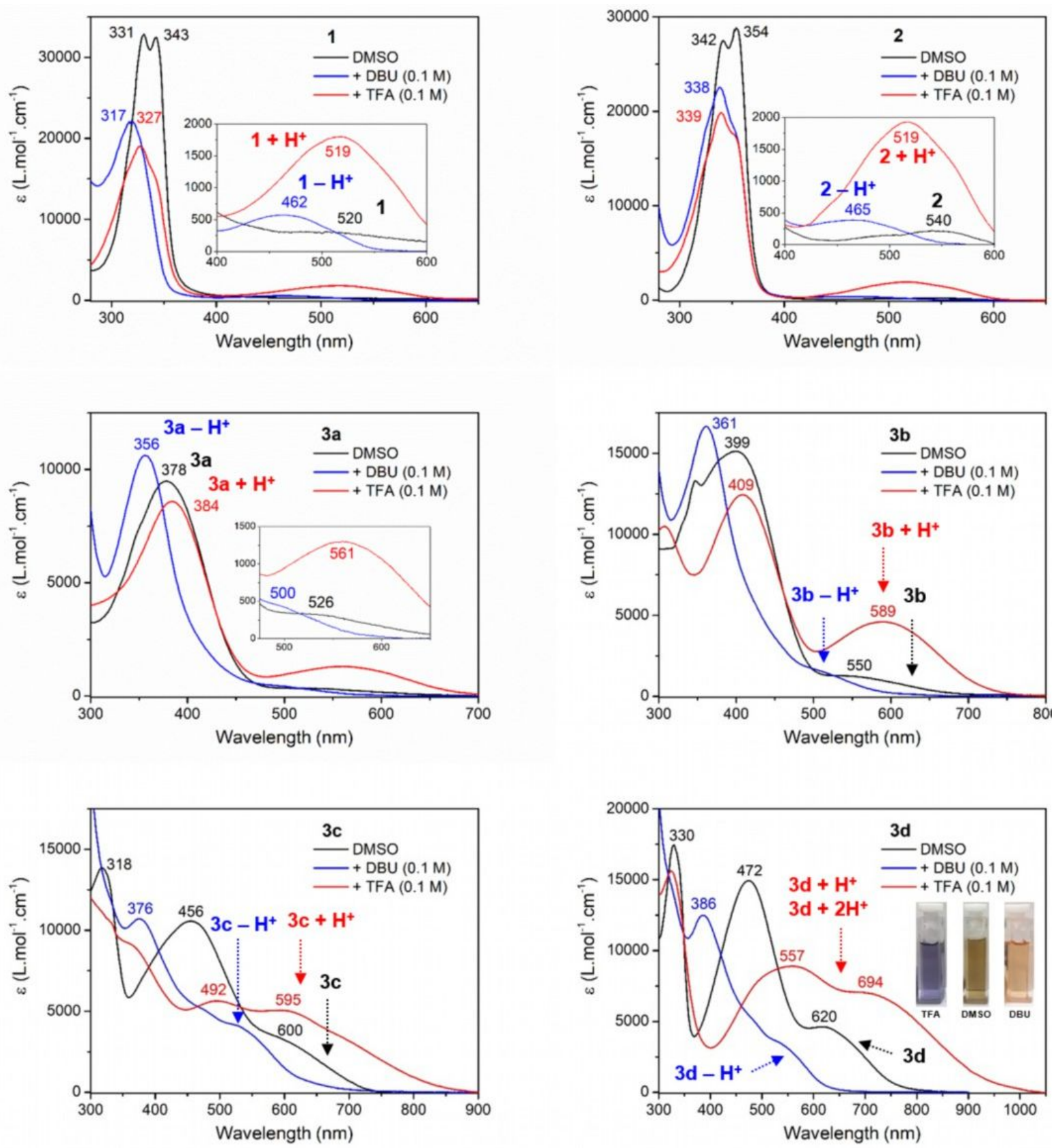

Figure S 33. pH-dependent absorption spectra of compounds 1, 2 and 3a-d in DMSO without or with presence of $0.1 \mathrm{M}$ trifluoroacetic acid (TFA) or $0.1 \mathrm{M}$ 1,8-diazabicyclo[5.4.0]undec-7-ene (DBU). 

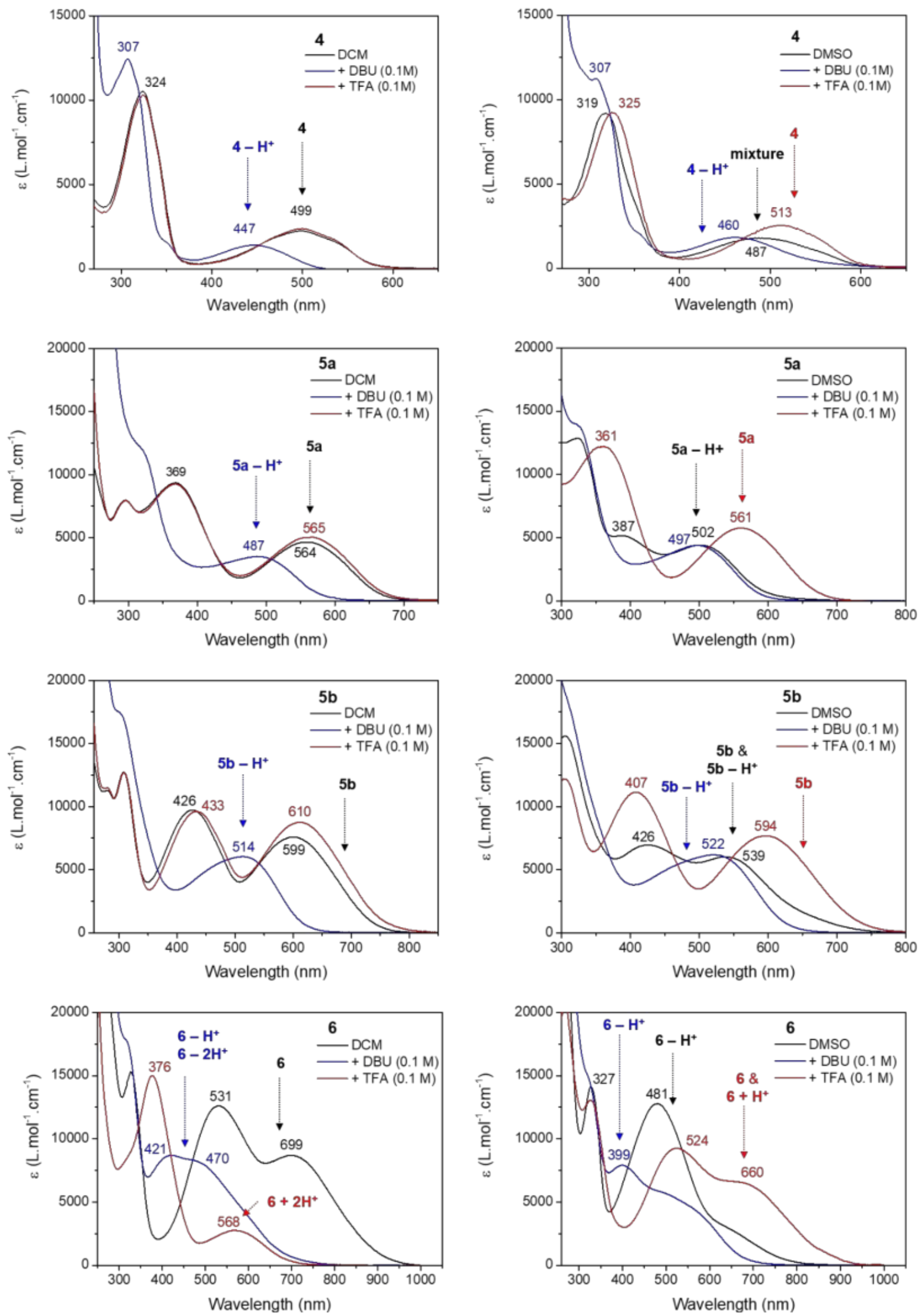

Figure S 34. pH-dependent absorption spectra of compounds $\mathbf{4}, \mathbf{5 a}, \mathbf{b}$ and $\mathbf{6}$ in DCM or DMSO without or with presence of $0.1 \mathrm{M}$ trifluoroacetic acid (TFA) or $0.1 \mathrm{M} \mathrm{1,8-diazabicyclo[5.4.0]undec-7-ene} \mathrm{(DBU).}$ 


\section{UV-VIS ABSORPTION vs. pH TITRATIONS}

\section{- Compound 3a}
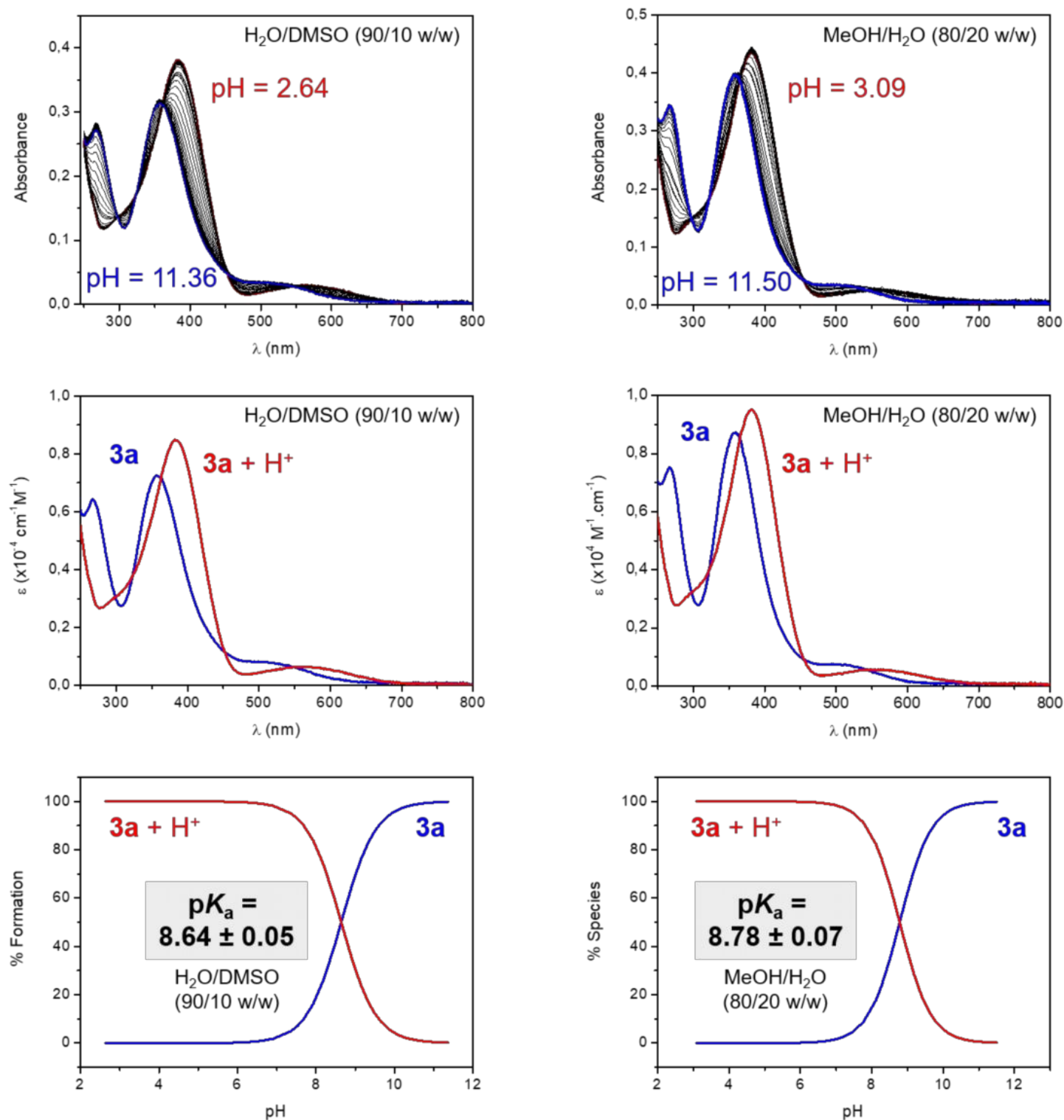

Figure S 35. Electronic absorption spectra vs. $\mathrm{pH}$ titration and distribution diagrams of the protonated species of 3a. Left column: Solvent: $\mathrm{H}_{2} \mathrm{O} / \mathrm{DMSO}(90 / 10 \mathrm{w} / \mathrm{w}) ; I=0.1 \mathrm{M}(\mathrm{NaCl}) ; T=25.0^{\circ} \mathrm{C}$; $[3 \mathrm{a}]=4.54 \times 10^{-5} \mathrm{M}$. Right column: Solvent: $\mathrm{MeOH} / \mathrm{H}_{2} \mathrm{O}(80 / 20 \mathrm{w} / \mathrm{w}) ; I=0.1 \mathrm{M}\left(n \mathrm{NBu}_{4} \mathrm{ClO}_{4}\right) ; T=25.0^{\circ} \mathrm{C} ;[3 \mathrm{a}]=4.66 \times 10^{-5} \mathrm{M}$.<smiles>C#[PH+]C1=CC(=Nc2ccccc2)C(Nc2ccccc2)=CC1=O</smiles>

3a<smiles>O=C1C=C(O)C(=Nc2ccccc2)C=C1Nc1ccccc1</smiles>

$3 \mathrm{a}+\mathrm{H}^{+}$

Scheme S 1. Structure of the protonated form of $\mathbf{3 a}$. 


\section{- Compound 3d}
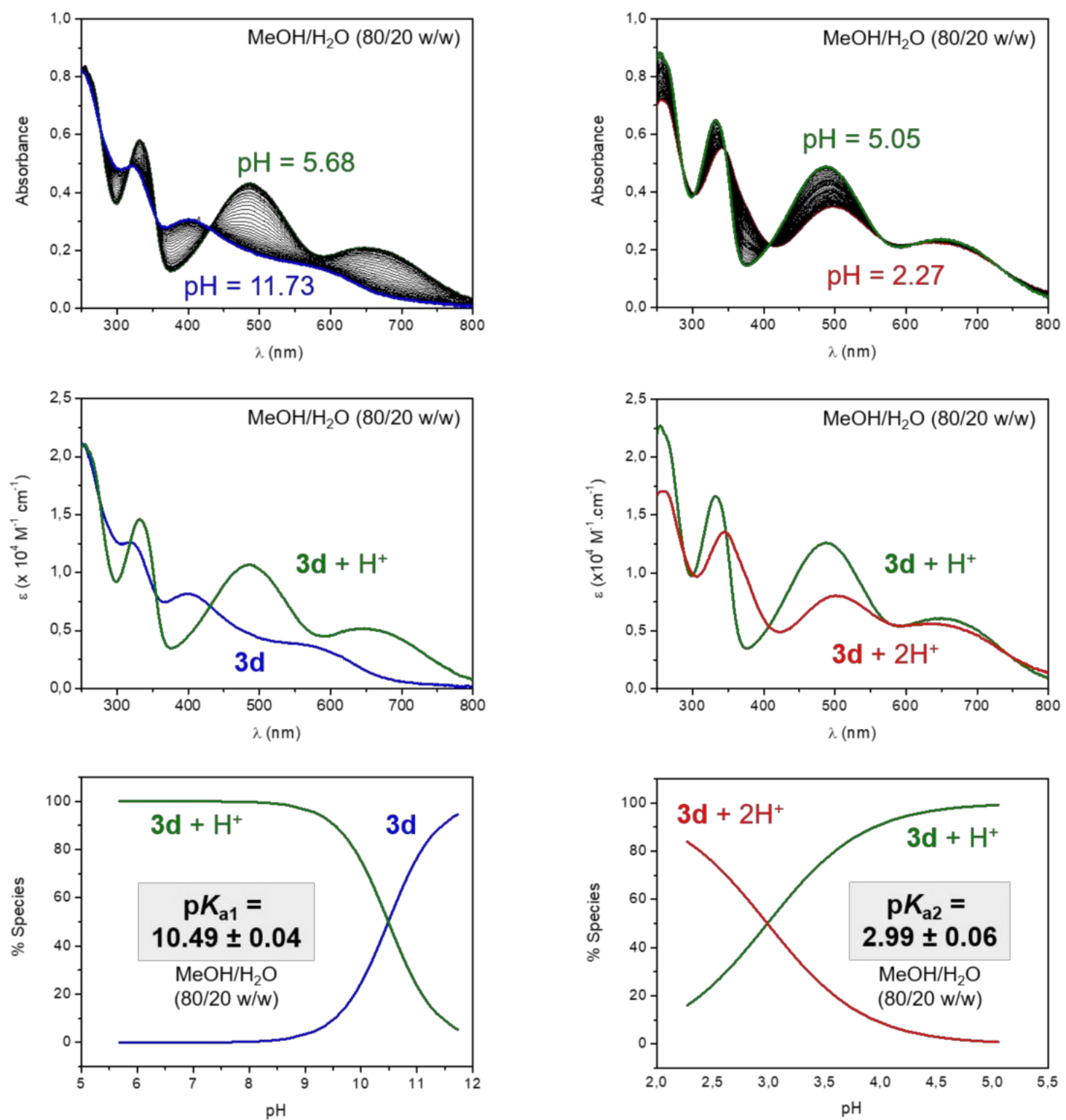

Figurfe S 36. Electronic absorption spectra vs. pH titration and distribution diagrams of the protonated species of 3d. Solvent: $\mathrm{MeOH} / \mathrm{H}_{2} \mathrm{O}(80 / 20 \mathrm{w} / \mathrm{w}) ; I=0.1 \mathrm{M}\left(n \mathrm{NBu}_{4} \mathrm{ClO}_{4}\right) ; T=25.0^{\circ} \mathrm{C}$; for $2.27<\mathrm{pH}<5.05$, [3d] = $4.03 \times 10^{-5} \mathrm{M}$ and for $5.68<\mathrm{pH}<11.73$, [3d] $=3.96 \times 10^{-5} \mathrm{M}$.

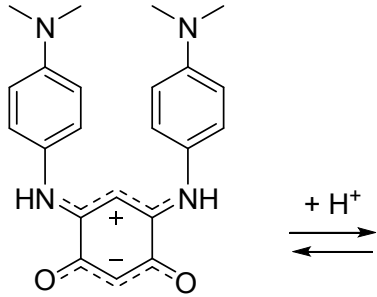

3d<smiles>CN(C)c1ccc(NC2=CC(Nc3ccc(N(C)C)cc3)C(=O)C=C2O)cc1</smiles>

$3 \mathrm{~d}+\mathrm{H}^{+}$<smiles></smiles>

$3 \mathrm{~d}+2 \mathrm{H}^{+}$

Scheme S 2. Structure of the protonated form of $\mathbf{3 d}$. 


\section{THEORETICAL CALCULATIONS}

Computational details. All DFT calculations are performed with Gaussian16 (a03p version), ${ }^{12}$ using default procedures and algorithms except for those noted below. Geometric and energy convergences are set to a tight threshold with the application of an ultrafine integration grid for $\mathrm{E}_{\mathrm{xc}}$. Ground-state geometries are optimized with the def2-TZVPP atomic basis set and the absence of imaginary frequencies is controlled by analytical computation of the Hessian at the same level of theory. TimeDependent (TD) calculations use the aug-cc-pVTZ (AVTZ) atomic basis set. The condensed phase studies are performed using the Polarizable Continuum Model (PCM), an implicit solvatation model that does not explicitly account for the specific interactions between the solvent and the solute molecules, the latter being placed inside a cavity. As the specific interactions between the solute and the solvent molecules are mostly hydrogen bonds, using the PCM is often a valid approximation for aprotic solvents.

Post-HF computations of the ground state energies [CCSD $(\mathrm{T})]$ and excited state (ES) properties [CC2] are performed using Turbomole (version 7.11$)^{13}$ with defaults procedures and algorithms. For each calculations, the frozen core approximation is used and CC2 computations are carried on using the Resolution of the Identity (RI) approximation, this mathematical approximation uses auxiliaries basis set on all atoms to reduce the computation cost of the integral calculation between the MO. ${ }^{14}$ Finally, the Dalton program (2017.alpha version) ${ }^{15}$ is used for the $\operatorname{CCSDR}(3)$ and CC3 calculations, again applying defaults procedures and convergence thresholds.

As described in the following paragraphs, we use a combination of post-HF and DFT computations to reach the theoretical best estimate (TBE) for the relative free energies $(G)$ of the various tautomers and vertical transitions energies for the absorption ( $\mathrm{E}^{\mathrm{VT}-\mathrm{a}}$ ), see Eq. 1, Eq. 2 and Eq. 3. Following the benchmark step, the free energy of each tautomers is estimated ( $\mathrm{G}^{\mathrm{TBE}}$ ) from the computation of the total electronic energy at the CCSD(T)/AVDZ level of theory corrected thanks to Eq. 1.

Moreover, as the solvent electronic density is locally coupled to the solute's electronic structure (in its vicinity), the changes in this density during a vertical transition have an impact on the solvent local polarization. Accurately determining the polarization response of the solvent helps reaching a better accuracy on the transition energies and can be accomplished with continuum models as well. ${ }^{16}$ During a vertical transition, only the electrons of the solvent do adapt to the new electronic density of the solute. For such cases, the non-equilibrium PCM limit is best suited and is therefore selected throughout. Subject to the nature of the electronic vertical transition to be modelled, different methods can in addition be applied to determine the vertical transition energies in solution. The Linear Response (LR) gives a correction related to the transition dipole moment $\mu_{12}$, and consequently $L R$ is adequate for bright transitions. ${ }^{17}$ The corrected Linear Response (cLR) provides a correction proportional to $\Delta \mu$, the difference of the total dipoles of the ES and the GS, a value that is large for charge-transfer (CT) transitions. ${ }^{18}$ The corrections made by these methods can be added together following the Eq. 2 where $\omega_{0}$ is the transition energy obtained through TD-DFT calculations where the solvent does not experience any changes in polarity during the transition, i.e., where the solvent is frozen in the GS configuration. In the studied series, several state switching occur between condensed and gas phase, making the state 
identification almost infeasible (but for the lowest transition) and $L R+c L R$ energies difficult to obtain. Thus, only the LR correction is taken into account for the excited-states above $S_{1}$. This is not a problem, as it is typically only the lowest ES that has a significant CT character. It was found during the benchmark process that the studied functionals are giving non-accurate transitions energies and that $\mathrm{CC} 2$ is providing much more accurate estimates. The vertical transitions are therefore computed at the CC2/AVTZ level of theory in gas phase and the solvent effects are determined with TD- M06-2X/AVTZ calculations (see Eq. 3)

$$
\begin{aligned}
& G^{\mathrm{TBE}}=E_{\text {gas }}^{\mathrm{CCSD}(\mathrm{T}) / \mathrm{AVDZ}}+\left[G_{\mathrm{PCM}}^{\mathrm{B} 3 \mathrm{YP} / \mathrm{def} 2-\mathrm{TZVPP}}-E_{\mathrm{PCM}}^{\mathrm{B} 3 \mathrm{YP} / \mathrm{def} 2-\mathrm{TZVPP}}\right]+\left[E_{\mathrm{PCM}}^{\mathrm{M} 06-2 \mathrm{X} / \mathrm{AVTZ}}-E_{\text {gas }}^{\mathrm{M} 06-2 \mathrm{X} / \mathrm{AVTZ}}\right] \\
& \text { Eq. } 1 \\
& E^{\mathrm{LR}+\mathrm{cLR}}=E^{\mathrm{LR}}+E^{\mathrm{cLR}}-\omega_{0}
\end{aligned}
$$

Eq. 2

$$
E^{\mathrm{VT}-\mathrm{a}}=\left[E_{\mathrm{gas}}^{\mathrm{CC} 2 / \mathrm{AVTZ}}-E_{\mathrm{gas}}^{\mathrm{M} 06-2 \mathrm{X} / \mathrm{AVTZ}}\right]+E^{\mathrm{LR}+\mathrm{cLR} / \mathrm{AVTZ}}
$$

Eq. 3

Benchmark. Direct comparison of DFT results to experimental data is not straightforward in our case as the possible existence of a Z/Q equilibrium is deduced from the recorded UV-visible spectra but their relative energies in several media remain unknown experimentally, and reproducing such spectra by computational chemistry is not straightforward. ${ }^{19}$ Post-HF methods can produce accurate data, yet they are extremely costly in terms of computational time, the use of DFT and related methods is compulsory to treat large compounds. As a series of homologous systems is considered here, a systematic study of a model system is carried out, using, on the one hand, a pathway of systematic improvement allowing to get accurate results with post-HF methods and, on the other hand, a statistical approach to analyze DFT results obtained with several functionals. This process should lead to an adequate choice of functional giving suitable results. Compound $\mathbf{1}$ is used as model since the other dyes are obtained by modification of this core. Based on the provided experimental data, a first guess is that 1 could be present in two stable tautomers, i.e., $Z$ and $Q$ forms, along with an Anionic (A) and Cationic (C) forms, obtained from protonation or deprotonation processes. A benchmark is carried on and focuses on three key properties (i) the relative energies of the zwitterionic and canonical structures, (ii) the optimized geometries of the GS for each of the structures, (iii) the vertical transition characteristics. As the tautomeric equilibrium $(Z / Q)$ is of large importance, reaching a good accuracy on the relative free energies of these two species is required.<smiles>N=CC1=CC(=O)C(O)=CC1=O</smiles>

a<smiles></smiles>

Z<smiles></smiles>

C $\stackrel{1}{\mathrm{H}}$<smiles></smiles>

A<smiles>C[As]1OB(O)SS(=O)O1</smiles>

Figure S 37. Most stable conformers of 1. (LEFT) Schemes highlighting the intramolecular hydrogen bonds in the most stable structure. (RIGHT) Numbering used as reference for the measurement of the interatomic distances, nitrogen atoms are depicted in blue and oxygens in red, all the bonds explicitly shown are used in the statistical analysis. 
As a first step, different possible conformers (depending on the orientation of the intra-molecular hydrogen bond) for each members of the series are optimized by DFT (PBE0/def2-TZVPP) and the absence of imaginary frequencies is checked. The structures sketched in Figure S 37 are kept for the following study as they present no imaginary frequencies and are the most stable conformers found. A batch of five "popular" exchange-correlation functionals are used in our benchmark: PBE0, ${ }^{20}$ B3LYP, ${ }^{21}$ M06-2X, ${ }^{22}$ CAM-B3LYP, ${ }^{23}$ and $\omega B$ 97X-D, ${ }^{24}$ that is carried on in gas phase, in order to reduce the computational cost for post-HF methods and to limit the deviation of the results inherent to the various solvent models and parameters used in different computing softwares.

\section{- Energies}

A comparative study is carried on the total (electronic) energy difference between the zwitterionic and canonic forms, optimized by using a post-HF method (CC2/def2-TZVPP, with the frozen core approximation). The geometry of the canonical structure is non-planar after computation of the frequencies, however, since the difference in total energy between planar and non-planar geometry is trifling $\left(<0.01 \mathrm{kcal}^{\mathrm{mol}}{ }^{-1}\right)$, the following study is carried on the planar structure. Computations of the total energy of these systems rely on the AVTZ atomic basis set with both post-HF [CC2, MP2, CCSD, and $\operatorname{CCSD}(\mathrm{T})]$ and DFT methods; the results are compiled in Table $S 2$.

Table S 2. Total energies for the zwitterionic (Z) and canonical (Q) tautomers of 1. Energy computations performed with the AVTZ basis set (except when noted for one case) and on the CC2/def2-TZVPP geometry, post-HF methods rely on the frozen core approximation. $\Delta Z / Q$ is given in $\mathrm{kcal}^{\mathrm{mol}}{ }^{-1}$.

\begin{tabular}{|c|c|c|c|}
\hline METHOD & Z (U.A.) & Q (U.A.) & $\Delta_{\mathbf{Z} / \mathbf{Q}}$ \\
\hline MP2 & -491.502204152 & -491.505992303 & -2.377 \\
\hline $\mathrm{CC} 2$ & -491.540413656 & -491.538657684 & 1.102 \\
\hline CCSD & -491.513077817 & -491.520151231 & -4.439 \\
\hline $\operatorname{ccsD}(T)$ & -491.615315582 & -491.621047802 & -3.597 \\
\hline $\operatorname{ccsD}(\mathrm{T}) / A V D Z$ & -491.014116060 & -491.020039908 & -3.717 \\
\hline PBEO & -491.822560696 & -491.821517716 & 0.654 \\
\hline B3LYP & -492.381615388 & -492.380030684 & 0.994 \\
\hline M06-2X & -492.180972732 & -492.184124277 & -1.978 \\
\hline CAM-B3LYP & -492.153844350 & -492.153630549 & 0.134 \\
\hline 凡B97X-D & -492.203875194 & -492.203269576 & 0.380 \\
\hline
\end{tabular}

The best accuracy that could be reach is given by the $\operatorname{CCSD}(T)$ "golden standard", giving the $Q$ tautomer to be more stable than its $Z$ counterpart by $3.6 \mathrm{kcal}^{\mathrm{mol}}{ }^{-1}$ (we recall: in gas phase). In DFT, the only consistent result is obtained by the use of the M06-2X functional giving the $Q$ structure to be more stable than the $Z$ one by $2 \mathrm{kcal}^{\mathrm{mol}}{ }^{-1}$. This difference between the $\operatorname{CCSD}(\mathrm{T})$ method and the M06-2X functional $\left(1.6 \mathrm{kcal}^{\mathrm{mol}}{ }^{-1}\right)$ is high enough to consider that the use of the $\operatorname{CCSD}(\mathrm{T})$ method could be mandatory for the determination of the $Z / Q$ equilibrium. As $\operatorname{CCSD}(T)$ calculation are very demanding, the use of AVDZ basis set is also tested with this method and it turns out that this double- $\zeta$ basis is sufficient to provide 
an accurate $\Delta Z / Q$ as the difference in the $Z / Q$ equilibrium between $\operatorname{CCSD}(T) / A V T Z$ and $C C S D(T) / A V D Z$ is as small as $0.1 \mathrm{kcal} . \mathrm{mol}^{-1}$ for the same system.

\section{- Geometries}

A statistical study of ten interatomic distances is performed on the optimized geometries of the four forms of 1 sketched in Figure $S 37$. Along with the geometric optimization, the frequencies are computed in order to confirm that the optimized geometry correspond to a true minimum. As references, the geometries optimized at the MP2/def2-TZVPP level (with the frozen core approximation) are used as this approach delivers very accurate geometries, often outperforming both CC2 and CCSD. ${ }^{25}$ Using the same def2-TZVPP basis set, the DFT geometries obtained with the studied set of functionals are upraised. The functional discrimination is carried on using usual descriptive statistical tools: the Mean Absolute Error (MAE) and the Root Mean Square (RMS). The statistical results are shown in Table $S$ 3.

Two main conclusions emerge. First, all five exchange-correlation functional deliver accurate results with RMS smaller than $0.01 \AA$. Second, B3LYP delivers the smallest MAE and RMS. As this holds for all four structures considered, B3LYP is selected for the ground-state optimization as well as for the determination of the vibrational frequencies. With the frequency computations, it is possible to correct the total energy $E^{\text {elec }}$ with thermodynamic contributions which mostly stems for the vibrational contributions, to estimate Gibbs energies. Therefore in the following, these corrections are determined at the B3LYP level.

Table S 3. Statistical analysis of the interatomic distances within 1 in gas phase. Ten interatomic distances (C-X with $X=C, O$ or $N$ ) are studied for the four forms depicted on Figure $S 37$. The presented values of MAE and RMS are obtained with respect to MP2[FC]/def2-TZVPP optimized geometries.

\begin{tabular}{r|lllll} 
Functional & B3LYP & PBE0 & M06-2X & CAM-B3LYP & $\omega B 97 X-D$ \\
\hline MAE & 0.005 & 0.007 & 0.009 & 0.009 & 0.008 \\
RMS & 0.006 & 0.008 & 0.010 & 0.010 & 0.010
\end{tabular}

\section{- Vertical transitions}

All computations (post-HF and TD-DFT methods) of vertical excitation energies are performed on the PBE0/def2-TZVPP optimized geometry. The excited states of interest are deduced from a comparison between the experimental UV-vis spectra and TD-DFT results, obtained with the AVTZ basis set. This first step is necessary to reduce the cost of the post-HF computation as one targets only the states of interest. At this stage, the anionic structure is excluded from the vertical transitions computation as it is not possible to deduce from the TD-DFT calculations a limited number of vertical transitions to be investigated. For the three other structures, two vertical transitions are selected, the first and either the third $(\mathrm{Q}$ and $\mathrm{C})$ or the fourth one $(\mathrm{Z})$, the latter being strongly dipole allowed and corresponding to the intense UV absorption band visible in the experimental spectra (see Figure S 31 and Figure S 33) References values for vertical transitions are computed at CC3/AVDZ and CCSDR(3) with double and triple $\zeta$ basis-set $s$ (AVDZ and AVTZ) levels of theory, which allows to approximate with a good accuracy 
the results that would have been obtained with CC3/AVTZ, denoted Theoretical Best Estimate (TBE) in the following (see Eq. 4). A statistical study of the transitions energies is performed between the results of lower cost theories (TD-DFT with several functionals and CC2) and the TBE references, using usual descriptive statistical tools [MAE, RMS and the Mean Squared Error (MSE)], the results are compiled in Table $\mathbf{S} 4$ and Table $\mathbf{S} 5$.

$$
E_{\mathrm{VT}-\mathrm{a}}^{\mathrm{TBE}} \equiv E^{\mathrm{CC} 3 / \mathrm{AVTZ}} \approx E^{\mathrm{CCSDR}(3) / \mathrm{AVTZ}}+\left(E^{\mathrm{CC} 3 / \mathrm{AVDZ}}-E^{\mathrm{CCSDR}(3) / \mathrm{AVDZ}}\right)
$$

Eq. 4

Table S 4. Vertical transitions of 1 and determined TBE. The vertical transitions are computed in gas phase. All values are in $\mathrm{eV}$, except when noted.

\begin{tabular}{ccccccc} 
& & \multicolumn{2}{c}{ AVDZ } & AVTZ & \multicolumn{2}{c}{ TBE } \\
\cline { 2 - 7 } & $\mathbf{S}_{\mathbf{n}}$ & $\mathbf{C C 3}$ & $\mathbf{C C S D R ( 3 )}$ & $\mathbf{C C S D R ( 3 )}$ & $\mathbf{e V}$ & $\mathbf{n m}$ \\
\hline $\mathrm{Z}_{\mathbf{Z}}$ & $\mathrm{S}_{1}$ & 2.171 & 2.215 & 2.221 & 2.177 & 570 \\
& $\mathrm{~S}_{4}$ & 4.298 & 4.352 & 4.353 & 4.300 & 288 \\
& $\mathrm{~S}_{1}$ & 3.137 & 3.216 & 3.221 & 3.142 & 395 \\
$\mathbf{Q}$ & $\mathrm{S}_{3}$ & 4.645 & 4.706 & 4.699 & 4.637 & 267 \\
& & & & & & \\
$\mathbf{C}$ & $\mathrm{S}_{1}$ & 2.843 & 2.896 & 2.899 & 2.846 & 436 \\
& $\mathrm{~S}_{3}$ & 4.575 & 4.671 & 4.667 & 4.572 & 271
\end{tabular}

Table S 5. TD-DFT and CC2 vertical transitions energies of 1 and statistical analysis compared to the TBE The vertical transitions are computed in gas phase, with the AVTZ basis set.

\begin{tabular}{|c|c|c|c|c|c|c|c|}
\hline & $S_{n}$ & B3LYP & PBEO & M06-2X & CAM-B3LYP & $\omega B 97 X-D$ & $\mathrm{CC2}$ \\
\hline \multirow{2}{*}{ Z } & $S_{1}$ & 2.106 & 2.192 & 2.358 & 2.359 & 2.356 & 2.161 \\
\hline & $\mathrm{S}_{4}$ & 4.251 & 4.351 & 4.546 & 4.514 & 4.495 & 4.317 \\
\hline \multirow{2}{*}{$\mathbf{Q}$} & $S_{1}$ & 2.820 & 2.913 & 3.176 & 3.115 & 3.097 & 3.136 \\
\hline & $\mathrm{S}_{3}$ & 4.198 & 4.316 & 4.646 & 4.568 & 4.535 & 4.607 \\
\hline \multirow{2}{*}{ C } & $S_{1}$ & 2.665 & 2.743 & 2.928 & 2.889 & 2.882 & 2.874 \\
\hline & $\mathrm{S}_{3}$ & 4.170 & 4.292 & 4.659 & 4.579 & 4.545 & 4.583 \\
\hline \multicolumn{2}{|c|}{ MAE } & 0.244 & 0.166 & 0.107 & 0.091 & 0.098 & 0.018 \\
\hline \multicolumn{2}{|c|}{ MSE } & 0.083 & 0.041 & 0.018 & 0.014 & 0.014 & 0.000 \\
\hline \multicolumn{2}{|c|}{ RMS } & 0.288 & 0.203 & 0.135 & 0.120 & 0.119 & 0.020 \\
\hline
\end{tabular}

It turns out that the tested functionals give different results and can be classified into two groups as function of their percentage of exact exchange. B3LYP and PBE0 provide accurate transitions energies for the zwitterionic structure whereas M06-2X, CAM-B3LYP, and $\omega B$ 97X-D are more suited for the quinoidal and cationic structures. This is a nice illustration that it is challenging to obtain a uniform description of states of different natures with a single functional in TD-DFT. In addition, we are well aware that the computation of cyanine-like transitions with TD-DFT remains a challenge,${ }^{26}$ probably explaining the relative poor performances of TD-DFT here. In contrast, CC2 delivers an accurate and 
uniform description of all transitions for the three forms. This method clearly provides the smallest RMS $(0.022 \mathrm{eV})$ compared to the TBE. We therefore compute the electronic transitions with CC2 in the following. However, as condensed phase computations are not straightforward with post-HF methods, the solvent effect is accounted for as correction obtained through TD-DFT calculations performed at the M06-2X/AVTZ level of theory. This functional is selected because (i) it gives the most accurate results for the total energies ( $Z / Q$ equilibrium) (ii) it delivers a reasonable description of the excited states for all the structures.

\section{- Conclusions on the benchmark}

Several conclusions emerged from this benchmark step. First, different conformers could be envisaged for each investigated structure. The most stable ones (possessing no imaginary frequencies) are depicted in Figure S 37 and have been studied during the benchmarking process. Theses conformations for the core structure will be kept for the following of the work. Moreover, a unique functional cannot be used to compute all the properties as (i) B3LYP is found to provide the most accurate geometries and selected for this purpose, (ii) M06-2X is found to be the only one amongst the tested functionals giving the same sign for the relative $Z / Q$ energies as $\operatorname{CCSD}(T)$, with a relative difference of $1.6 \mathrm{kcal}^{\mathrm{mol}}{ }^{-1}$ between M06-2X and $\operatorname{CCSD}(\mathrm{T})$ relative energies, which is nevertheless too different to make M06-2X very reliable, and (iii) no functional gives sufficiently accurate results for the transition energies for all the structures, the $\mathrm{CC} 2$ method has to be used although solvent effects have to be accounted for at the TD-DFT level in practice.

In the following of this work, geometries are optimized at the B3LYP/def2-TZVPP level of theory, the $Z / Q$ equilibrium is estimated from total-energies computation with the $\operatorname{CCSD}(T)$ "golden standard" method corrected with the free energy corrections obtained at the B3LYP/def2-TZVPP level and solvent corrections calculated at the M06-2X/AVTZ level. The excited state energies are determined through CC2/AVTZ computations corrected for solvent effects obtained at the M06-2X/AVTZ level.

Theoretical study of all systems. The results of the computations concerning the tautomeric equilibria between the $Z$ and $Q$ structures along with their conformers are shown in Table $S$, the results of the computations concerning the vertical transitions energies and their features for the most stables conformers are summarized in Table $S 9$.

\section{- Detailed study of 1}

No significant differences are found between the DMF and DMSO solvents, in terms of energies, geometries, nor spectroscopic properties of the tautomers. Indeed, in each solvent, the Boltzmann distribution gives the zwitterionic structure to be the only form present with a $100 \%(\mathbf{1 Z})$ to $0 \%(\mathbf{1 Q})$ ratio, indicating that the observed difference in intensity within the absorption spectra when switching solvent is not due to a change of the proportion of the zwitterionic structure. Note that this differs from the gas phase, illustrating that, as expected, polar solvents do stabilize the zwitterionic form. 
The UV-vis transitions of interest mainly involve an excitation from the Highest Occupied Molecular Orbital (HOMO) or HOMO-1 to the Lowest Unoccupied Molecular Orbital (LUMO). As the vertical transition features are identical in both solvents, the electronic density differences (EDD) and the molecular orbitals (MO) of interest are plotted in DMSO only (Figure S 38). The cyanine-like structure of the zwitterionic form can be seen by the topology of the HOMO and HOMO-1, respectively centered on the oxygen (HOMO) and the nitrogen (HOMO-1) moieties which is chemically intuitive as the negative charge is borne by the oxygen-capped cyanine. The LUMO is located on the single $\mathrm{C}-\mathrm{C}$ bonds, meaning that they gain a double bond character upon excitation which is consistent with the coupling principle (see also the EDD in Figure S 38).

On the experimental UV-vis spectrum, two bands can be observed. The first one, around $450 \mathrm{~nm}$, exhibits a solvent-dependent intensity and position whereas the second one with a vibronic structure, at ca. $330-340 \mathrm{~nm}$, possesses both a high intensity. In agreement with previous studies, ${ }^{15}$ the longwavelength absorption can be attributed to the first transition of the zwitterionic structure. The intense UV absorption band is computed at relatively similar energies for both the zwitterionic and the canonical structures (see Table $S$ ) and corresponds to a $S_{0} \rightarrow S_{4}$ transition in the former. The computed transition $S_{0} \rightarrow S_{1}$ for the canonical structure at $428 \mathrm{~nm}$ is not observed in the experimental spectra, which is consistent with the absence of $1 Q$ tautomer in solution. In $1 Z$, the first transition $\left(S_{0} \rightarrow S_{1}\right)$ induces a strong decrease of the dipole moment upon excitation which fits the EDD where it is clear that the density change is significant for the first transition. This means that the first transition possesses a significant CT character with a CT distance reaching ca. $1 \AA$ according to Le Bahers' model. ${ }^{27}$ Moreover, the chemical intuition of the negative charge located around the oxygen atoms being displaced to the positive charge located around the nitrogen atoms is confirmed by the dipole orientation in both $S_{0}$ and $S_{1}$ of $\mathbf{Z Z}$ (see Table $S 7$ ). Despite these successes, we note that the computations are unable to reproduce the experimentally observed solvatochromism between DMF and DMSO, which we attribute to the limits of the PCM model.

Table S 6. Relevant excited states of $1 Z$ and $1 Q$ in DMF and DMSO. Geometries computed at the B3LYP/def2TZVPP level of theory, MO compositions are extracted from M06-2X/AVTZ results. Vertical transitions energies are calculated using Eq. 3. The Boltzmann distributions $\left(p_{i}\right)$ are calculated from the free energies obtained through Eq. 1.

\begin{tabular}{|c|c|c|c|c|c|c|c|}
\hline & & pi & Sn & $f$ & eV & $\mathrm{nm}$ & MO composition \\
\hline \multirow{4}{*}{ 옴 } & \multirow{2}{*}{$1 \mathrm{Q}$} & \multirow{2}{*}{$0 \%$} & $\mathrm{~S}_{1}$ & 0.041 & 2.895 & 428 & $H \rightarrow L(95 \%)$ \\
\hline & & & $\mathrm{S}_{3}$ & 0.561 & 4.444 & 279 & $\mathrm{H}-1 \rightarrow \mathrm{L}(93 \%)$ \\
\hline & \multirow{2}{*}{$1 Z$} & \multirow{2}{*}{$100 \%$} & $\mathrm{~S}_{1}$ & 0.005 & 2.279 & 544 & $\mathrm{H} \rightarrow \mathrm{L}(98 \%)$ \\
\hline & & & $\mathrm{S}_{4}$ & 0.601 & 4.004 & 310 & $\mathrm{H}-1 \rightarrow \mathrm{L}(97 \%)$ \\
\hline \multirow{4}{*}{$\sum_{\Delta}^{L}$} & \multirow{2}{*}{$1 \mathrm{Q}$} & \multirow{2}{*}{$0 \%$} & $S_{1}$ & 0.041 & 2.895 & 428 & $H \rightarrow L(95 \%)$ \\
\hline & & & $\mathrm{S}_{3}$ & 0.565 & 4.350 & 285 & $\mathrm{H}-1 \rightarrow \mathrm{L}(93 \%)$ \\
\hline & \multirow{2}{*}{$1 Z$} & \multirow{2}{*}{$100 \%$} & $\mathrm{~S}_{1}$ & 0.005 & 2.276 & 545 & $\mathrm{H} \rightarrow \mathrm{L}(98 \%)$ \\
\hline & & & $\mathrm{S}_{4}$ & 0.604 & 4.001 & 310 & $\mathrm{H}-1 \rightarrow \mathrm{L}(97 \%)$ \\
\hline
\end{tabular}


Table S 7. Dipole moment of the GS and key ES for the tautomers of $\mathbf{1}(\mathbf{1 Z}$ and $1 \mathrm{Q})$ in DMSO. The density analysis is performed at the M06-2X/AVTZ level using the PCM to model the solvent. $\mu, D$ and QP are the dipole moment, the CT distance and the CT charge, respectively. The two latter are computed using Le Bahers' model. The $\mu$ vector is scaled by 0.5

\begin{tabular}{|c|c|c|c|c|c|c|}
\hline & \multicolumn{3}{|c|}{$1 Q$} & \multicolumn{3}{|c|}{$1 Z$} \\
\hline & & $\mathbf{S}_{1}$ & $\mathbf{S}_{3}$ & $\begin{array}{l}S_{0} \\
\end{array}$ & $\mathbf{S}_{1}$ & S \\
\hline$\mu(D)$ & 5.53 & 8.18 & 3.87 & 11.38 & 8.32 & 12.23 \\
\hline$D(\AA)$ & I & 0.93 & 0.89 & I & 1.09 & 0.49 \\
\hline QP (e) & I & 0.63 & 0.42 & I & 0.56 & 0.36 \\
\hline
\end{tabular}
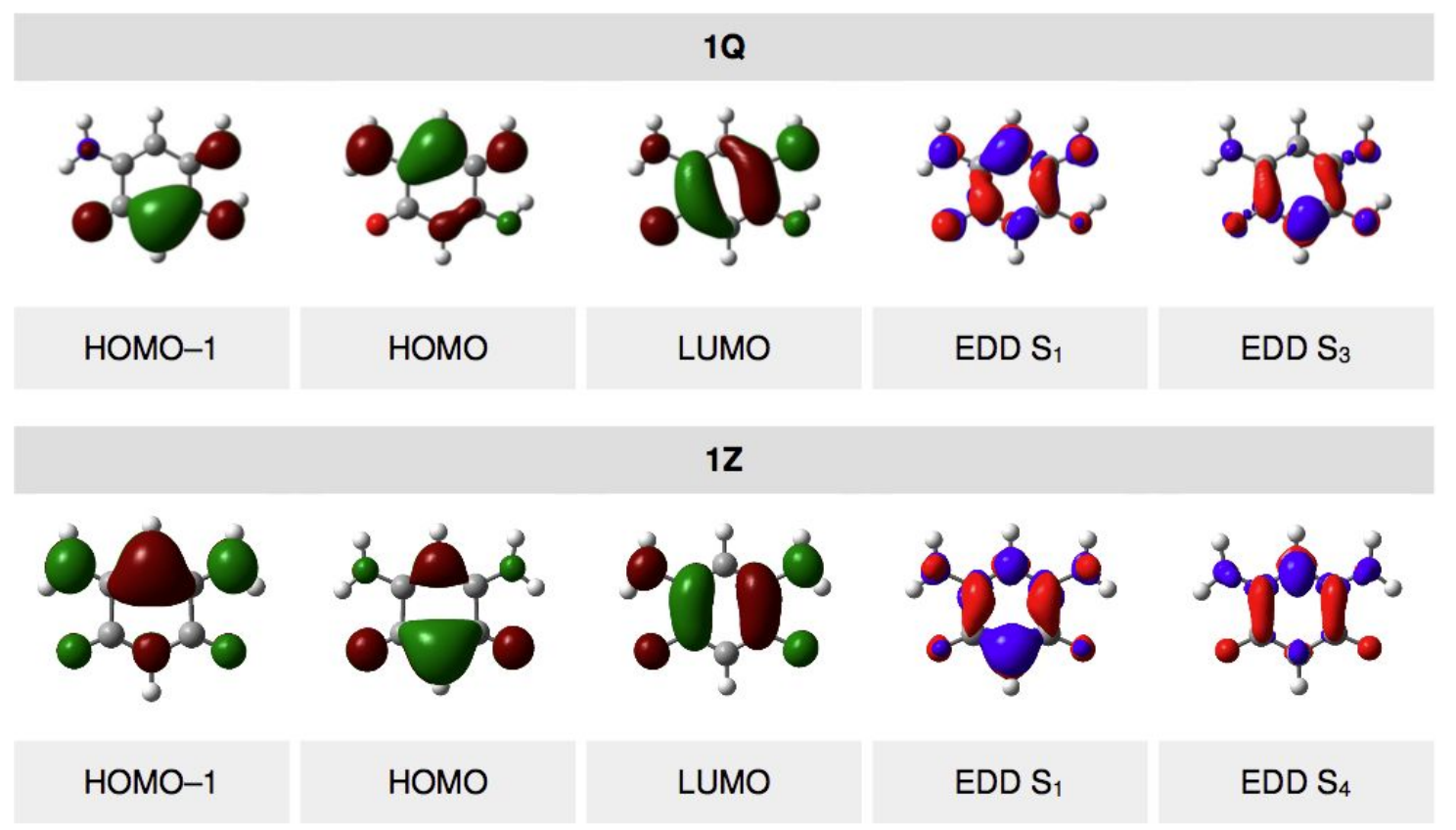

Figure S 38. MO and EDD plots for $1 \mathrm{Q}$ and $1 \mathrm{Z}$ in DMSO. Calculations performed at the M06-2X/AVTZ level using the PCM as solvatation model. MO isovalue: $0.04 \mathrm{au} \& E D D$ isovalue: $0.003 \mathrm{au}$. The red and blue lobes of the $E D D$ indicate increase and decrease of the electron density upon absorption, respectively.

\section{- Conformation and tautomeric equilibrium in $2-3 x$}

First, as the degrees of freedom for long alkyl chains are too numerous to obtain a complete picture of the conformational space, the butyl substituents of $\mathbf{2}$ are replaced by methyl groups in order to reproduce the electronic (inductive) effects of such substitution. The inductive effects are rather accurately simulated by this approximation and thus the variation of the transitions energies are expected to be reliable.

Given the previously established most stable conformers for 1 , two conformers per structure can be envisaged for 2 , depending on the relative orientation of the methyl groups' hydrogens. The optimized geometries, independently of the starting point, yields the same structures where the methyl groups are 
staggered compared to the central hydrogen atom (see Figure $S 39$ ). For the anionic structure, the interaction of the methyl group with the oxygen atom leads to a structure less stable than when the methyl group is oriented as displayed in Figure S 39. The addition of methyl groups does not modify the relative stabilities of the $\mathbf{Z}$ and $\mathbf{Q}$ conformers, as the Boltzmann distribution still gives $100 \%$ of the $\mathbf{Z}$ form.

For $\mathbf{3 a}$, considering the $\pi-\pi$ interactions of the phenyl groups, two orientations can be envisaged for the side groups: one where the phenyl rings are parallel to each other, and another with a perpendicular orientation, named slip-shaped (s) and t-shaped ( $\mathrm{t}$ ), respectively. For each configuration, the phenyls are oriented at $60^{\circ}$ from the $[N, N, O, O]$-core at the start of the optimization process. After the optimization, a slight stabilization is observed for the $t$ structures compared to their s counterparts (see Figure $S 40$ ). The addition of phenyl groups on the nitrogen-capped cyanine generates a small difference in the relative stabilities of the $\mathbf{Z}$ and $\mathbf{Q}$ forms, in favor of the latter as compared to $\mathbf{1}$. However, the zwitterionic form remains strongly dominant and only the vertical transitions of the most stable conformer (3aZt) are studied in the following.

For $\mathbf{3 c}$ and $\mathbf{3 d}$, the addition of amino groups in the para position of the phenyls slightly modifies the Boltzmann distribution for $t$ and $s$ shaped conformers (see Figure $S 41$ and Figure $S 42$ ), however the zwitterionic forms are always found to be most stable compared to the canonical ones and for all compounds, the $t$ shaped zwitterion is found to be the most present one in solution (noted $3 c \mathbf{z t}$ and $3 \mathrm{dZt}$ respectively).

As a more technical note, we underline that the $\operatorname{CCSD}(T)$ computations were not carried on for $\mathbf{3 b}$ as (i) more than 8 conformers exists per $Q$ and $Z$ forms for this molecule (ii) CCSD(T)/AVDZ computations are very time-consuming, and (iii) the substituents variations have no impact on the tautomeric equilibria for the other molecules and thus, it is unlikely that the -OMe substituent would modify the equilibrium, especially because the non-substituted and amino-substituted phenyl yield the same conclusions. 
Table S 8. Tautomeric equilibria for the investigated conformers of each structures of the $[N, N, O, O]-$ series in DMSO. The $\Delta G$ in $\mathrm{kcal} / \mathrm{mol}$ and the Boltzmann distributions $\left(p_{i}\right)$ are calculated from the free energies (G $\left.{ }^{T B E}\right)$ obtained through Eq. 1. Note that for $3 d$, the free energies are determined using CCSD instead of CCSD(T) in Eq. 1 , as the latter method is beyond reach for this large compound.

\begin{tabular}{|c|c|c|c|}
\hline & Conformer & $\Delta \mathbf{G}$ & $\mathbf{p}_{\mathrm{i}}$ \\
\hline \multirow{2}{*}{1} & Z & 0.000 & $100 \%$ \\
\hline & Q & 3.982 & $0 \%$ \\
\hline \multirow{2}{*}{2} & Z & 0.000 & $100 \%$ \\
\hline & Q & 6.741 & $0 \%$ \\
\hline \multirow{4}{*}{$3 a$} & $\mathrm{Zt}$ & 0.000 & $80 \%$ \\
\hline & Zs & 0.914 & $17 \%$ \\
\hline & Qt & 2.301 & $2 \%$ \\
\hline & Qs & 2.509 & $1 \%$ \\
\hline \multirow{4}{*}{$3 c$} & $\mathrm{Zt}$ & 0.000 & $88 \%$ \\
\hline & Zs & 1.185 & $12 \%$ \\
\hline & Qt & 4.104 & $0 \%$ \\
\hline & Qs & 4.044 & $0 \%$ \\
\hline \multirow{4}{*}{$3 d$} & $\mathrm{Zt}$ & 0.000 & $71 \%$ \\
\hline & Zs & 0.517 & $29 \%$ \\
\hline & Qt & 3.715 & $0 \%$ \\
\hline & Qs & 3.860 & $0 \%$ \\
\hline
\end{tabular}

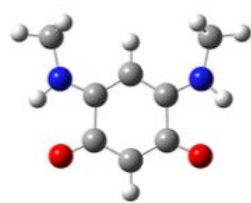

Z $100 \%$

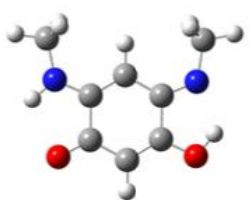

Q

$0 \%$

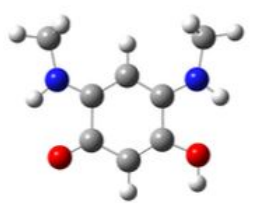

C

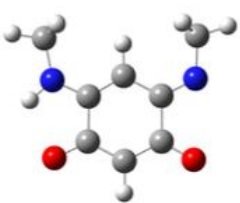

A

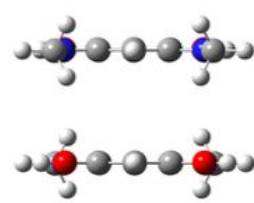

Z lateral view

Figure S 39. Representation of the four forms of 2. The Boltzmann distributions are obtained through Eq. 1. The lateral view of $\mathbf{2 Z}$ shows the staggered conformation of the methyl groups. 


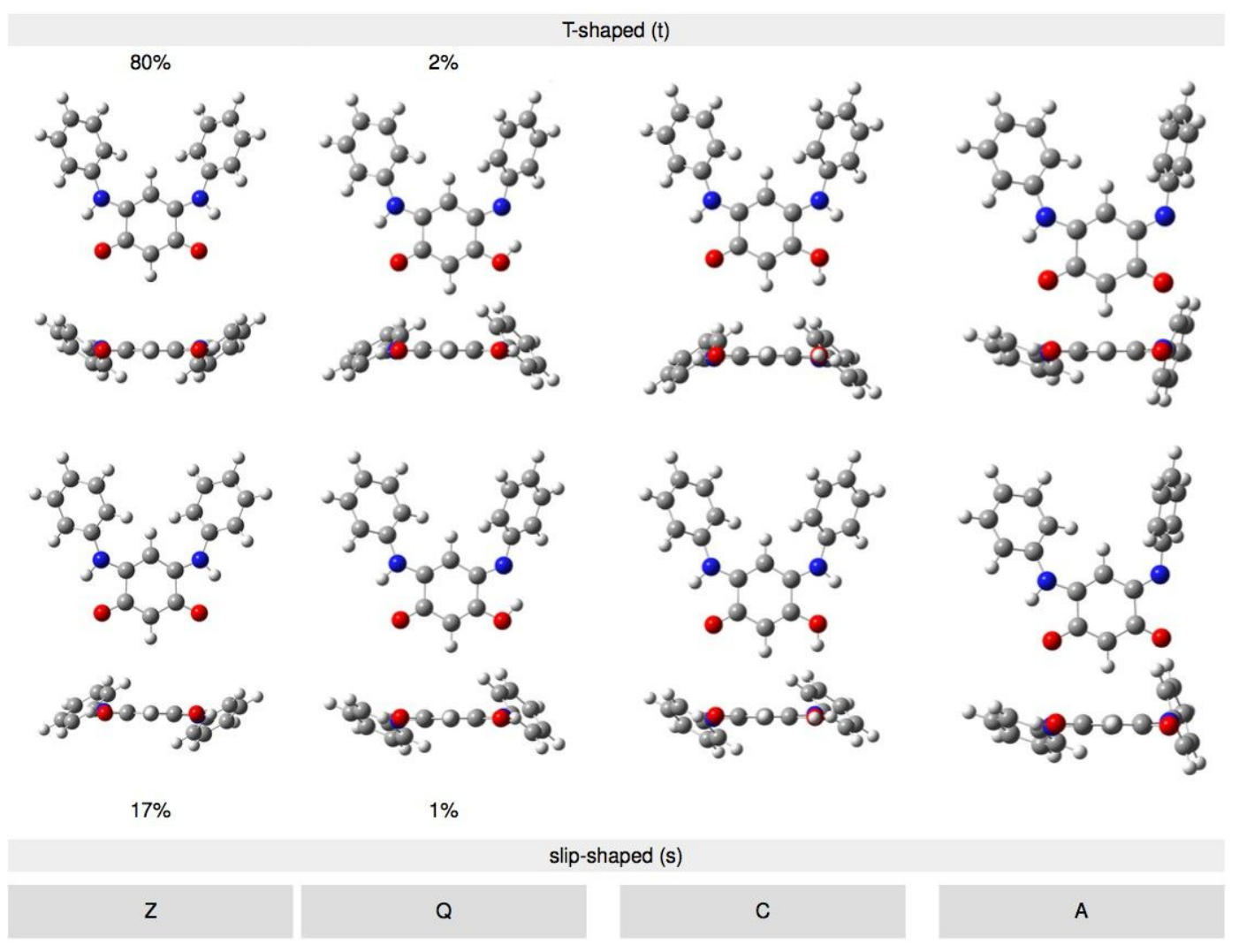

Figure S 40. 3D representation of 3a along with their respective Boltzmann distributions. (UP) view from above. (BOTTOM) front view. Geometries computed at the B3LYP/def2-TZVPP level in DMSO (PCM). In the $t$-shaped zwitterionic compound (the most stable one), dihedral angles of $35.6^{\circ}$ and $-37.1^{\circ}$ between the core and the $N$ substituted phenyls are measured.

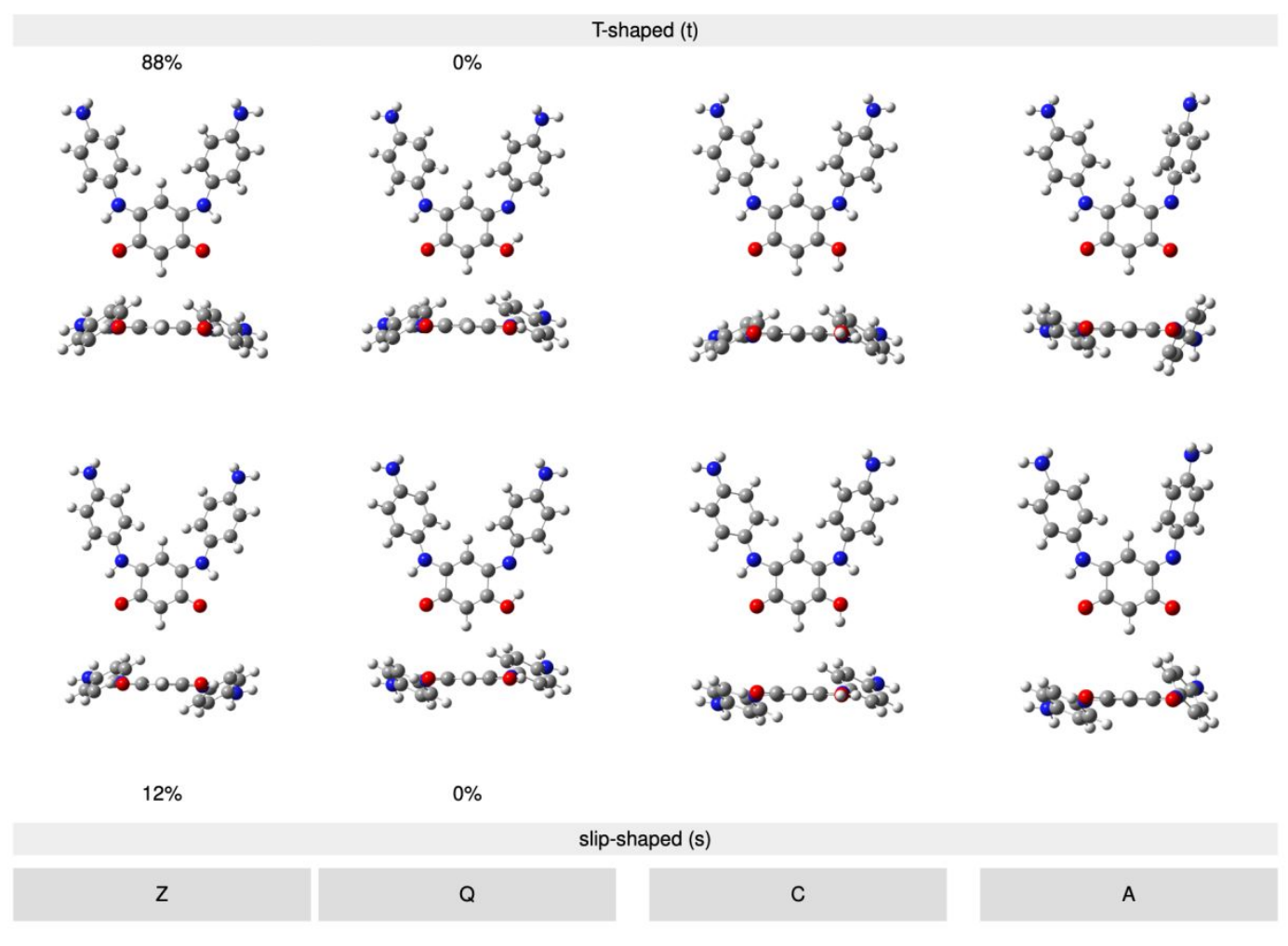

Figure S 41. 3D representation of 3c along with their respective Boltzmann distributions. In the t-shaped zwitterionic compound (the most stable one), dihedral angles of $32.2^{\circ}$ and $-35.1^{\circ}$ between the core and the $\mathrm{N}$-substituted phenyls are measured. See caption on Figure S 40 on page 40 for details. 


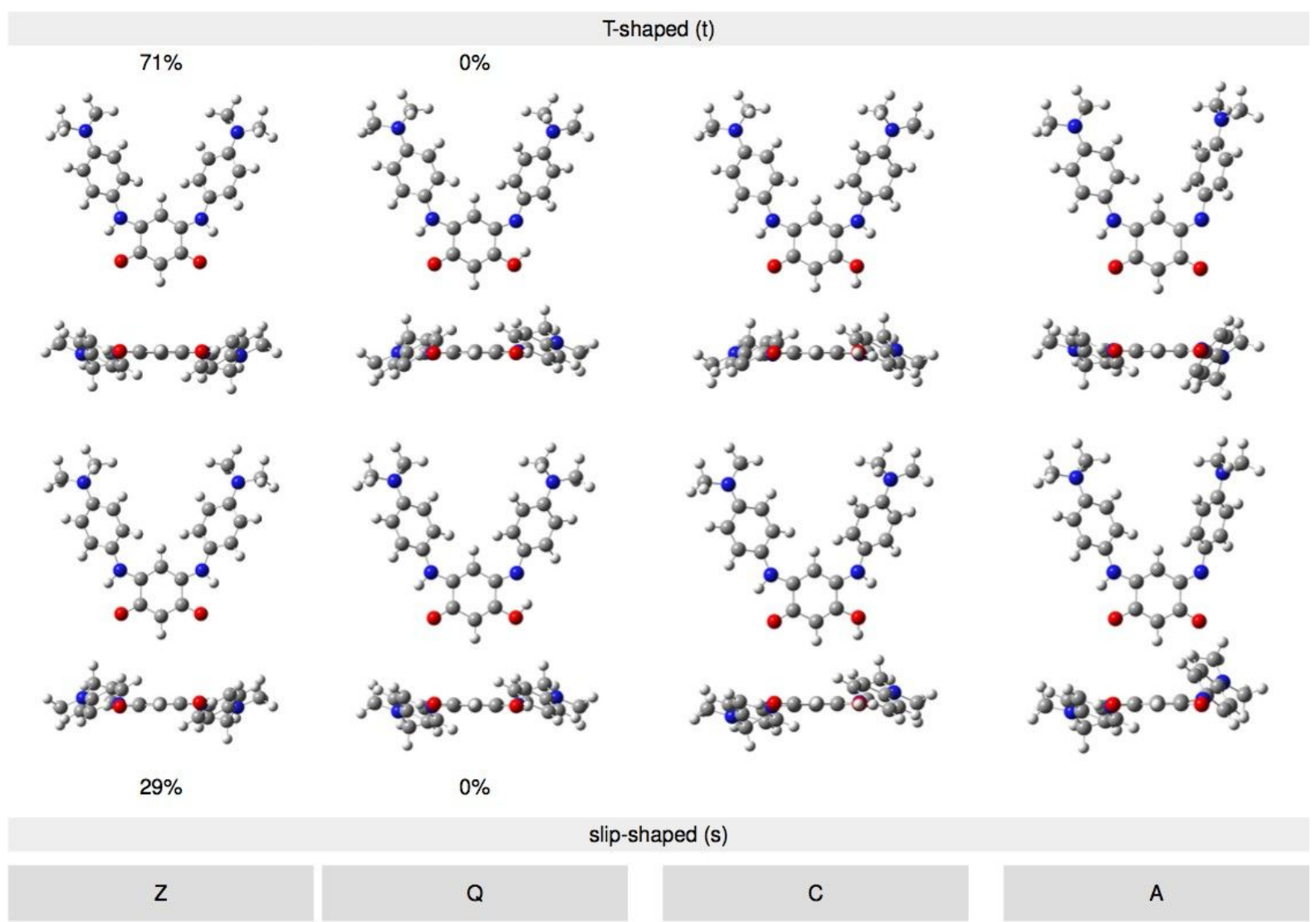

Figure S 42. 3D representation of $3 \mathbf{d}$ along with their respective Boltzmann distributions. In the t-shaped zwitterionic compound (the most stable one), dihedral angles of $29.9^{\circ}$ and $-32.5^{\circ}$ between the core and the $\mathrm{N}$-substituted phenyls are measured. See caption on Figure S 40 on page 40 for details.

\section{- Substitution effect on the absorption properties of (zwitterionic) $2-3 x$}

Going from 1 to 2 , the experimental spectrum shows a redshift of the intense UV absorption band whereas the second band, around ca. $505 \mathrm{~nm}$ is slightly blue-shifted. The computations reproduce these effects for both transitions, see Table S 9. The addition of methyl groups does not change the general repartition of the electronic density over the molecule as can be seen on the MO and EDD plots (see Figure S 43): the HOMO-1, HOMO, and LUMO are very similar to those of the non-alkylated counterpart. Likewise the characters of the transitions are similar, the first one $\left(S_{0} \rightarrow S_{1}\right)$ exhibiting a significant CT character in $\mathbf{2 Z}$ as in $\mathbf{1 Z}$ (see Table $S$ 9). The EDD pictures show that the nitrogen-capped cyanine part acts an acceptor in the $\mathrm{S}_{0} \rightarrow \mathrm{S}_{1}$ transition and a donor in the $\mathrm{S}_{0} \rightarrow \mathrm{S}_{4}$ transition, in both $\mathbf{1 Z}$ and $\mathbf{2 Z}$. Indeed, the stabilization of the positive charge of the $\mathrm{N}$-capped cyanine induced by the inductive effect of the alkyl groups should make the $\mathrm{N}$-capped cyanine less willing to accept incoming electronic density and thus destabilizing the $\mathrm{S}_{1}$. With the same reasoning, the $\mathrm{N}$-capped cyanine being a donor in the $S_{0} \rightarrow S_{4}$ transition, its increase of electronic density would make it more willing to give a part of its electronic density and thus stabilizing the $\mathrm{S}_{4}$ when comparing $\mathbf{1 Z}$ and $\mathbf{2 Z}$.

The phenyl's substitution (3a) leads to clear modifications of the UV-vis spectrum, both experimentally and theoretically. The intense band of 1 with a clear vibronic aspect around $340 \mathrm{~nm}$ becomes structureless and redshifted to ca. $380 \mathrm{~nm}$. The long-wavelength absorption band (around $500-520 \mathrm{~nm}$ ) is slightly more intense in $\mathbf{3 a}$ than in $\mathbf{1}$. This transition presents, as in $\mathbf{1 Z}$, a pure HOMO to LUMO 
transition. These two orbitals are faintly delocalized on the phenyl groups in $\mathbf{3 a Z t}$ and their general topology are alike the ones of $\mathbf{1 Z}$ and $\mathbf{2 Z}$. Consequently, the EDD of $\mathrm{S}_{1}$ in $\mathbf{3 a Z t}$ is close to the one of $\mathbf{Z Z}$ (see Figure $S 44$ ). As highlighted in the Table $S 9$, this transition possesses almost the same CT characteristics in $\mathbf{3 a Z t}$ and in $\mathbf{1 Z}$, indicating that the phenyl substitution has a limited impact on the transition energy, consistent with the measurements. In fact, the experimental UV-vis spectra show a small displacement of the long-wavelength absorption band from $\mathbf{1 Z}$ to $3 \mathrm{aZ} t$ to lower energies $\left({ }^{1} \lambda_{\max }^{\text {expt. }}=\right.$ $\left.518 \mathrm{~nm} \rightarrow{ }^{3 a} \lambda_{\max }^{\text {expt. }}=536 \mathrm{~nm}\right)$ which is corroborated by the computations $\left({ }^{1} \lambda_{\max }^{\text {th. }}=544 \mathrm{~nm} \rightarrow{ }^{3 \mathrm{a}} \lambda_{\max }^{\text {th. }}=\right.$ $582 \mathrm{~nm}$ ). In 1Z, the only UV transition possessing a significant oscillator strength is the $S_{0} \rightarrow S_{4}$ excitation with $f=0.601$. In contrast, in $3 \mathrm{aZt}$, two transitions are found to possess large oscillator strengths in the UV domain, the $S_{0} \rightarrow S_{2}$ and $S_{0} \rightarrow S_{5}$ transitions with respectively $f=0.772$ and $f=$ 0.345. These transitions mainly involve transitions from the HOMO-1 $\left(\mathrm{S}_{2}\right)$ or the HOMO-2 $\left(\mathrm{S}_{5}\right)$ orbitals, which are significantly delocalized on the phenyl rings, to the LUMO, which mostly conserves its topology with contributions on the $\mathrm{C}-\mathrm{C}$ single bonds of the core. These UV transitions in 3aZt present strong CT characters, which is obviously different from the non-substituted scenario.

The addition of methoxy moieties in para-position of the phenyls leads in $\mathbf{3 b}$ to an overall increase of the absorption along with a bathochromic shift of the bands (compared to $\mathbf{3 a}$ ) in the recorded UV-vis spectra up to $399 \mathrm{~nm}$ and $550 \mathrm{~nm}$ for the second and first bands respectively. As for 1, 2 and 3a, the $\mathrm{S}_{0} \rightarrow \mathrm{S}_{1}$ involves predominantly the HOMO and LUMO orbitals, however the latter is less pure in terms of composition than in the previous dyes $(78 \% \mathrm{H} \rightarrow \mathrm{L}$ and a slight $(20 \%) \mathrm{H}-1 \rightarrow \mathrm{L}$ contributions). The LUMO is still located on the $\mathrm{C}-\mathrm{C}$ single bonds of the 6 -carbon ring of the $[N, N, O, O]$-core, meaning that the bis-cyanines nature pertains (see Figure $S 45$ ). The HOMO in $\mathbf{3 b Z t}$ _ee however is largely delocalized on the methoxy-phenyls substituents and on the nitrogen-capped cyanine. The HOMO-1 is located on the oxygen-capped cyanine. The EDD plot shows that the $S_{0} \rightarrow S_{1}$ transition still induces a gain of electronic density on the single $\mathrm{C}-\mathrm{C}$ bonds of the $[N, N, O, O]$-core, together with a loss of density on the cyanines central carbons for this first band, we note that the methoxy-phenyl groups do not significantly contribute to the topology of the transition. The addition of donating groups (-OMe) destabilizes the charge borne by the nitrogen-capped cyanine, thus the latter is less likely to accept electronic density coming from the oxygen-capped cyanine. The stabilization effect can be seen on the weakening of the CT character (see Table S 9) with the diminution of all three $\Delta \mu$, D and QP compared to $3 a$.

The recorded UV-vis spectra of $3 c$ with amino substituents shows three absorption bands possessing no vibronic features. Compared to $\mathbf{3 a}$, the bands are displaced to lower energies to such an extent that two absorption bands are now located in the visible region, at $456 \mathrm{~nm}$ and $600 \mathrm{~nm}$, the latter being more intense than $\mathbf{3 a}$. As for $\mathbf{3 b}$, the computed lowest transition in $\mathbf{3 c}\left(\mathrm{S}_{0} \rightarrow \mathrm{S}_{1}\right)$ still involves predominantly the HOMO and LUMO orbitals but also comprises a small HOMO-2 to LUMO contribution (32\%). The bis-cyanine nature persists as highlighted by the LUMO still centered on the single $\mathrm{C}-\mathrm{C}$ bonds of the central $[O, O, N, N]$-core (see Figure $S 46$ ). The HOMO-2 is centered on the oxygen-capped cyanine, presenting a nice analogy in its topology to the HOMO in $\mathbf{1 Z}$. As in the previous dyes, the EDD plot shows a gain of electronic density on the single $\mathrm{C}-\mathrm{C}$ bonds of the $[O, O, N, N]$-core along with a loss of 
density on both of the cyanines central carbons. However, the changes on the oxygen atoms become completely negligible. Like in $\mathbf{3 b}$, the amino-phenyl groups do not significantly contribute to the transition according to our calculations. As for $\mathbf{2}$ and $\mathbf{3 b}$, the addition of donor groups $\left(-\mathrm{NH}_{2}\right)$ stabilizes the positive charge borne by the nitrogen-capped cyanine. Here, the stabilization effect is more intense than in $\mathbf{3 b}$ probably because the addition of amino functions changes the nature of the transition: unlike the previous molecules in the $[N, N, O, O]$-series, this transition does not possesses a CT character in $\mathbf{3 c Z t}$ with $D=0.19 \AA$ (see Table S 9). There is likely a kind of compensation between the $C T$ internal to the zwitterion and the amino-to-core $\mathrm{CT}$. This band is indeed not very solvatochromic experimentally. This decrease of the CT character is also consistent with the increase of the oscillator strength of the transition, noticed experimentally.

In $\mathbf{3 d}$, as in $\mathbf{3 c}$, the three bands displayed on the UV-vis spectra do not shows vibronic features. Going from $3 \mathbf{c}$ to $3 \mathbf{d}$, the UV-vis spectrum in DMSO shows a redshift and an increase of the absorption with a maximum at $472 \mathrm{~nm}$ and $620 \mathrm{~nm}$ for the two bands in the visible region. Such changes are quite expected when using dialkylamino groups instead of amino groups. The first computed transition $\left(\mathrm{S}_{0} \rightarrow\right.$ $\mathrm{S}_{1}$ ) involves predominantly the HOMO and LUMO orbitals (70\%) but as in $3 \mathrm{c}$, also and the HOMO-2 to LUMO excitation $(27 \%)$. The LUMO presents a general topology close to the one of the previously studied dyes in the series, mostly located on the single $\mathrm{C}-\mathrm{C}$ bonds on the 6 -carbon ring of the central $[O, O, N, N]$-core, thus the bis-cyanine nature pertains in $\mathbf{3 d}$. Nevertheless, as in $\mathbf{3 c}$, the HOMO-2 is located on the oxygen-capped cyanine. Unlike in $3 c$ or in any of the other dyes, this transition presents not only a strong CT character in $\mathbf{3 d}$, with $D=1.22 \AA$, but is also accompanied by an increase of the dipole moment upon transition, i.e., one would expect positive solvatochromism to appear, which is the case for $\mathbf{3 d}$ experimentally and therefore clearly differs from the negative solvatochromism noted for, e.g, 1. We interpret this as a CT from the alkyl-amino groups towards the core of the dye rather than an "in-core" CT. This is visible by comparing the EDD plots of $\mathbf{3 a}, \mathbf{3 b}, \mathbf{3 c}$, and $\mathbf{3 d}$. 
Table S 9. Relevant excited states of the $Z$ conformers of $\mathbf{1}, \mathbf{2}, \mathbf{3} \mathbf{a}, \mathbf{3 b} \mathbf{b} \mathbf{3} \mathbf{c}$ and $\mathbf{3 d}$ in DMSO. Geometries computed at the B3LYP/def2-TZVPP level of theory, MO compositions are extracted from M06-2X/AVTZ results. Vertical transitions energies are calculated using Eq. 3 for $S_{1}$ and without the cLR correction for the others. $\mu, D$ and $Q P$ are the dipole moment, the CT distance and the CT charge, respectively. The two latter are computed using Le Bahers' model.

\begin{tabular}{|c|c|c|c|c|c|c|c|c|}
\hline & Sn & f & $E(e V)$ & $\mathrm{nm}$ & MO comp & $\Delta \mu(D)$ & $D(A)$ & QP (e) \\
\hline \multirow{2}{*}{$1 Z$} & $\mathrm{~S}_{1}$ & 0.005 & 2.279 & 544 & $H \rightarrow L(98 \%)$ & -3.07 & 1.090 & 0.585 \\
\hline & $\mathrm{S}_{4}$ & 0.601 & 4.004 & 310 & $\mathrm{H}-1 \rightarrow \mathrm{L}(97 \%)$ & 0.85 & 0.490 & 0.360 \\
\hline \multirow{2}{*}{$2 Z$} & $\mathrm{~S}_{1}$ & 0.001 & 2.377 & 522 & $H \rightarrow L(99 \%)$ & -3.13 & 1.118 & 0.582 \\
\hline & $\mathrm{S}_{4}$ & 0.654 & 3.900 & 318 & $H-1 \rightarrow L(98 \%)$ & 0.90 & 0.526 & 0.359 \\
\hline \multirow{2}{*}{$3 a Z t$} & $\mathrm{~S}_{1}$ & 0.011 & 2.130 & 582 & $H \rightarrow L(96 \%)$ & -3.83 & 1.362 & 0.585 \\
\hline & $\mathrm{S}_{2}$ & 0.772 & 3.262 & 380 & $\mathrm{H}-1 \rightarrow \mathrm{L}(95 \%)$ & 2.02 & 0.930 & 0.452 \\
\hline \multirow{2}{*}{ 3bZt_ee } & $\mathrm{S}_{1}$ & 0.034 & 2.159 & 574 & $\begin{array}{l}\mathrm{H}-1 \rightarrow \mathrm{L}(20 \%) \\
\mathrm{H} \rightarrow \mathrm{L}(78 \%)\end{array}$ & -2.58 & 1.026 & 0.534 \\
\hline & $\mathrm{S}_{2}{ }^{1}$ & 0.743 & & & $\begin{array}{l}H-1 \rightarrow L(78 \%) \\
H \rightarrow L(19 \%)\end{array}$ & 3.75 & 1.413 & 0.558 \\
\hline \multirow{2}{*}{$3 c Z t$} & $\mathrm{~S}_{1}$ & 0.091 & 2.130 & 582 & $\begin{array}{l}H-2 \rightarrow L(32 \%) \\
H \rightarrow L(66 \%)\end{array}$ & -0.43 & 0.185 & 0.480 \\
\hline & $\mathrm{S}_{2}$ & 0.610 & 2.856 & 434 & $\begin{array}{l}\mathrm{H}-2 \rightarrow \mathrm{L}(67 \%) \\
\mathrm{H} \rightarrow \mathrm{L}(31 \%)\end{array}$ & 3.11 & 1.094 & 0.594 \\
\hline \multirow{2}{*}{$3 d Z t$} & $\mathrm{~S}_{1}$ & 0.211 & 2.045 & 606 & $\begin{array}{l}\mathrm{H}-2 \rightarrow \mathrm{L}(27 \%) \\
\mathrm{H} \rightarrow \mathrm{L}(70 \%)\end{array}$ & 3.00 & 1.217 & 0.512 \\
\hline & $\mathrm{S}_{2}$ & 0.495 & 2.583 & 480 & $\begin{array}{l}\mathrm{H}-2 \rightarrow \mathrm{L}(71 \%) \\
\mathrm{H} \rightarrow \mathrm{L}(26 \%)\end{array}$ & 1.28 & 0.419 & 0.635 \\
\hline
\end{tabular}

\footnotetext{
1 Due to a strong mixing of states between TD-DFT and CC2 computations, it was impossible to accurately determine the energy of the transition.
} 


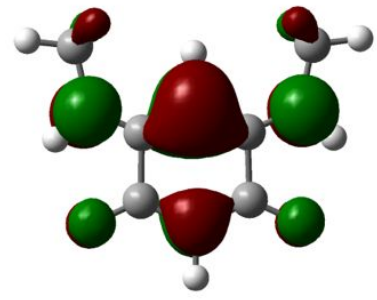

$\mathrm{H}-1(43)$

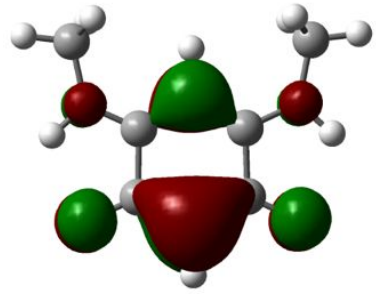

$\mathrm{H}(44)$

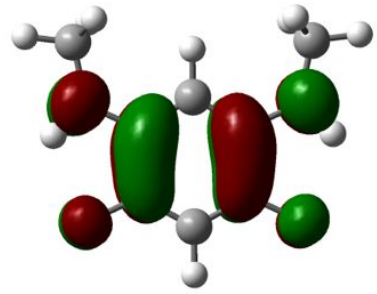

$L(45)$

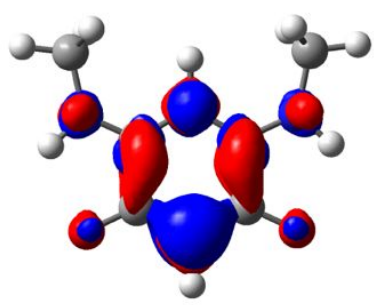

EDD $S 1$

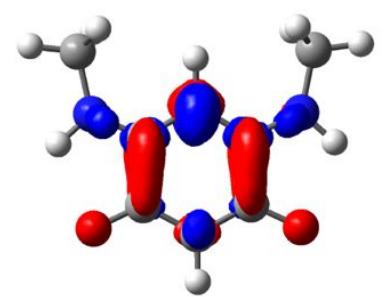

EDD S4

Figure S 43. MO and EDD plots for $\mathbf{2 Z}$ in DMSO. MO isovalue: 0.04 au \& EDD isovalue: 0.003 au. See caption of Figure $S 38$ on page 37 for other details. 


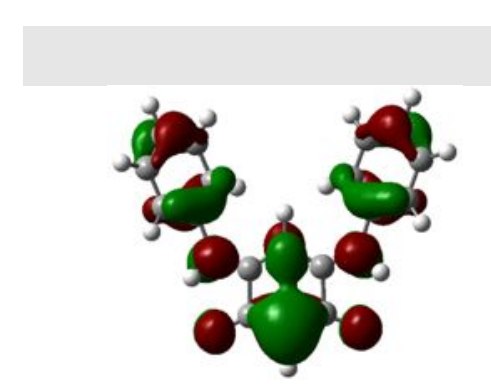

H-1 (75)

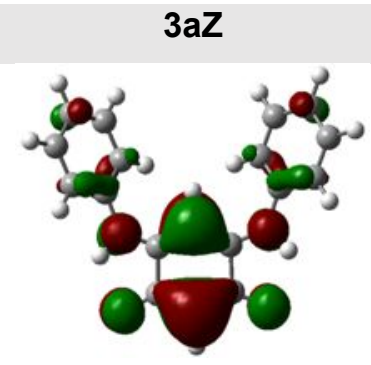

H (76)

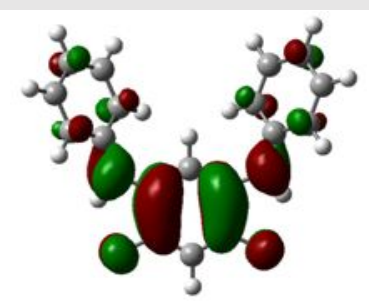

L (77)

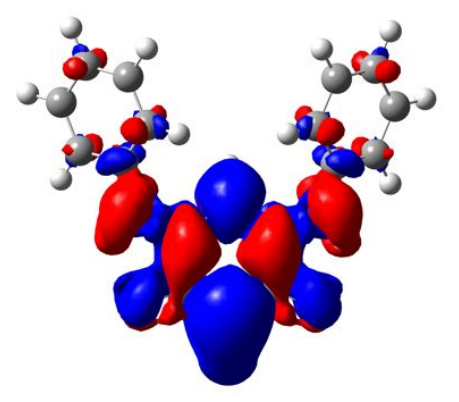

EDD $S 1$

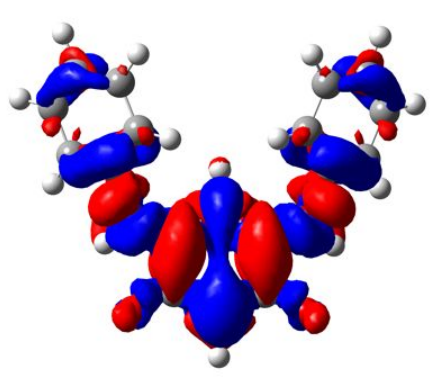

EDD $S 2$

Figure S 44. MO and EDD plots for 3aZ in DMSO. MO isovalue: 0.04 au \& EDD isovalue: 0.0006 au. See caption of Figure $S 38$ on page 37 for other details.

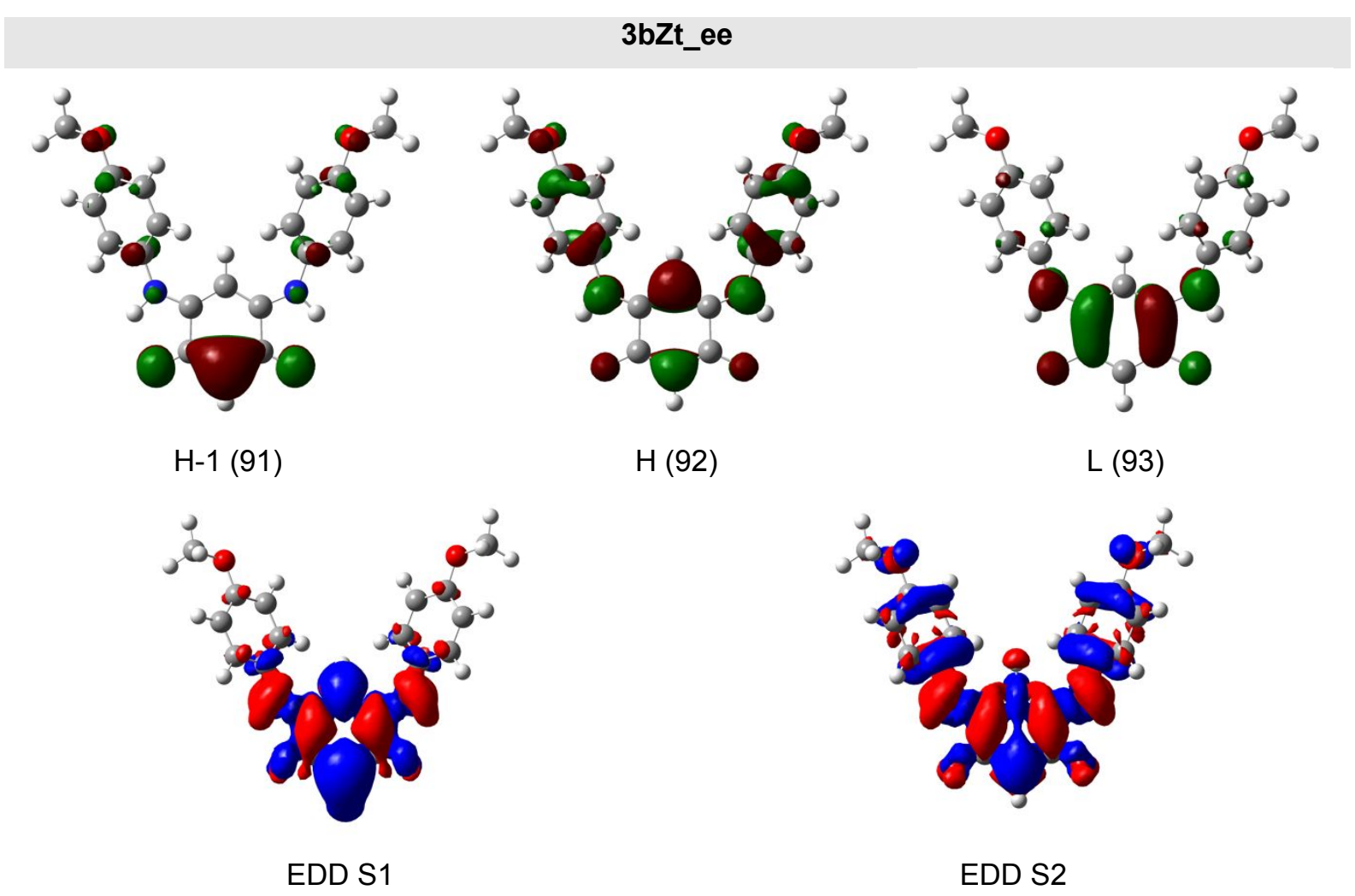

Figure S 45. MO and EDD plots for 3bZt_ee in DMSO. MO isovalue: 0.04 au \& EDD isovalue: 0.0006 au. See caption of Figure $S 38$ on page 37 for other details. 


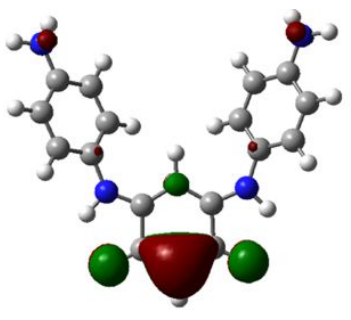

$\mathrm{H}-2(82)$

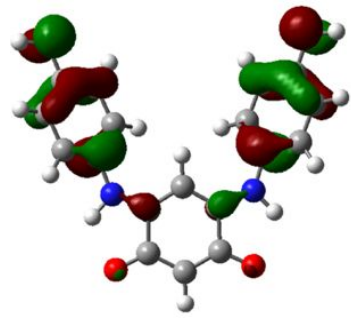

H-1 (83)

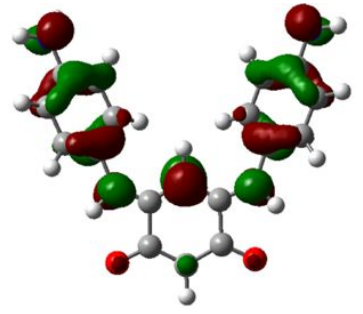

$H(84)$

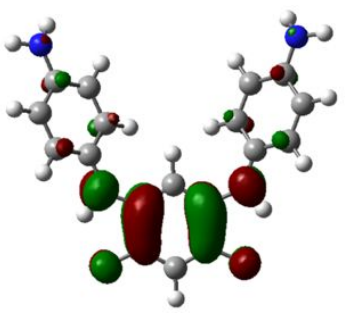

$L(85)$

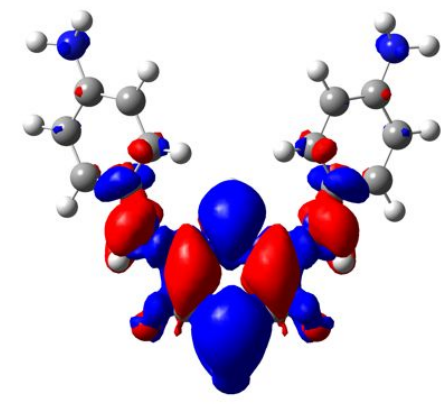

EDD S1

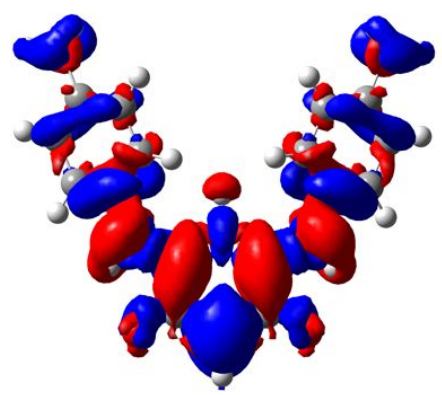

EDD S2

Figure S 46. MO and EDD plots for 3cZt in DMSO. MO isovalue: 0.04 au \& EDD isovalue: 0.0006 au. See caption of Figure $S 38$ on page 37 for other details.

$3 d Z t$

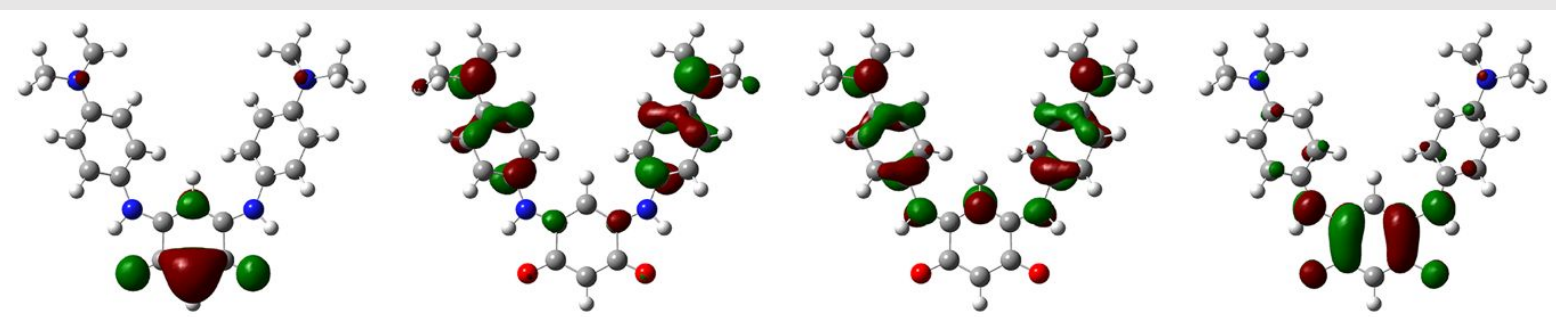

H-2 (98)

H-1 (99)

H (100)

L (101)

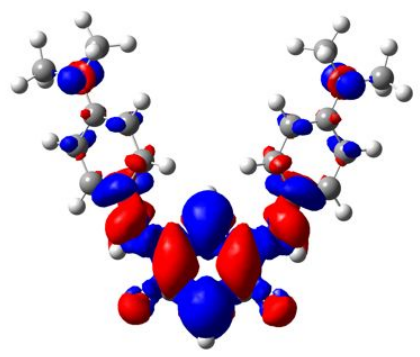

EDD S1

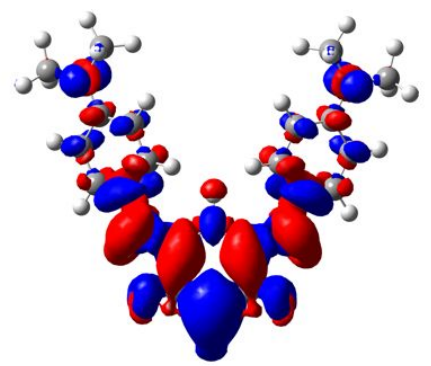

EDD S2

Figure S 47. MO and EDD plots for 3dZt in DMSO. MO isovalue: 0.04 au \& EDD isovalue: 0.0006 au. See caption of Figure $S 38$ on page 37 for other details. 


\section{Comparisons between neutral and cationic species}

To complete the previous investigation, we have also studied the spectral features of the cationic species and compared them to their neutral form. To this end, we focused on the $S_{0} \rightarrow S_{1}$ transitions only, which have been obtained using Eq. 3. In the present case, the Boltzmann populations between conformers were estimated using the following equation:

$$
G^{\mathrm{TBE}}=E_{\mathrm{gas}}^{\mathrm{CC} 2 / \mathrm{AVTZ}}+\left[G_{P C M}^{\mathrm{B} 3 \mathrm{LYP} / \mathrm{def} 2-\mathrm{TZVPP}}-E_{\mathrm{PCM}}^{\mathrm{B} 3 \mathrm{LP} / \mathrm{def} 2-\mathrm{TZVPP}}\right]+\left[E_{\mathrm{PCM}}^{\mathrm{M} 06-2 \mathrm{X} / \mathrm{AVTZ}}-E_{\mathrm{gas}}^{\mathrm{M} 06-2 \mathrm{X} / \mathrm{AVTZ}}\right]
$$

Eq. 5

In the case of the molecule 2, the experimental spectra recorded in DMSO+TFA shows a shift of the broad first absorption band towards higher energies compared to the neutral conditions : from $540 \mathrm{~nm}$ in DMSO to $519 \mathrm{~nm}$ in acidic medium (see Figure $S 48$ - top left). Indeed a charge transfer is more likely to occur in a zwitterionic structure (DMSO) than in a positively-charged molecule (DMSO+TFA). This displacement goes along with an increase of the intensity from ca. $200 \mathrm{~L}^{\mathrm{mol}}{ }^{-1} . \mathrm{cm}^{-1}$ (DMSO) to 2000 L. $\mathrm{mol}^{-1} . \mathrm{cm}^{-1}$ (DMSO+TFA). These experimental observations are nicely corroborated by the computed first transition of the putative cationic structure $\mathbf{2 C}$ in DMSO, which is slighted blue-shifted compared to the one of $\mathbf{2 Z}$ and, more importantly, shows a strong increase of the oscillator strength from 0.001 (2Z) to 0.051 (2C) (see Figure S 48 - middle top).

When going to the methylated cationic structure $\mathbf{4}$, we notice that the spectra of $\mathbf{2}$ and $\mathbf{4}$ in acidic medium exhibit very similar absorption band in the visible domain, however a displacement of this band toward higher energies is observed when going from an acidic to a neutral medium for 4 (see Figure S 48 bottom left). This unexpected behavior can be explained by the basic character of DMSO, which can be sufficient to deprotonate 4 , thus leading to the formation of a canonical $\mathbf{4}-\mathbf{H}^{+}$structure. This hypothesis is supported by the computational results: the first $\left(S_{0} \rightarrow S_{1}\right)$ transition computed in 4 is highly similar to the one computed for $\mathbf{2 C}$, and the first transition computed for the $\mathbf{4}-\mathbf{H}^{+}$structure (4Qa and $\mathbf{4 Q b}$ conformers) is displaced toward higher energies (see Figure $S 48$ - middle bottom).

For $\mathbf{3 a}$, one can note the same tendencies as for $\mathbf{2}$ when comparing neutral and acidic results with a blue-shift of the first absorption band and a strong increase of its intensity in DMSO+TFA (see Figure $S 49$ - top left). The simulated UV-vis spectra for the zwitterionic (3aZ) and the cationic (3aC) structures shows the same tendencies (see Figure $S 49$ - middle top). In this $\mathbf{3 a} / \mathbf{5 a}$ pair, one can also assume that the DMSO would be basic enough to deprotonate the methylated cation $5 \mathbf{a}$, leading to the formation a neutral form of the latter $\left(\mathbf{5 a}-\mathbf{H}^{+}\right)$in DMSO. Indeed, the experimental UV-vis in DMSO+TFA of $\mathbf{3 a}$ and $\mathbf{5 a}$ are almost equivalent, whereas a blue shift of the absorption is observed for $\mathbf{5 a}$ in (non-acidic) DMSO (see Figure $S 49$ - bottom left). Here again this hypothesis is supported by the calculation of the first transition in a neutral form of $\mathbf{5 a}(\mathbf{5 a Q})$, which is blue-shifted compared to the one of $\mathbf{5 a}$, the latter being similar to the one computed for $3 \mathrm{aC}$, in agreement with the experimental trends (see Figure $S 49$ - middle bottom). 


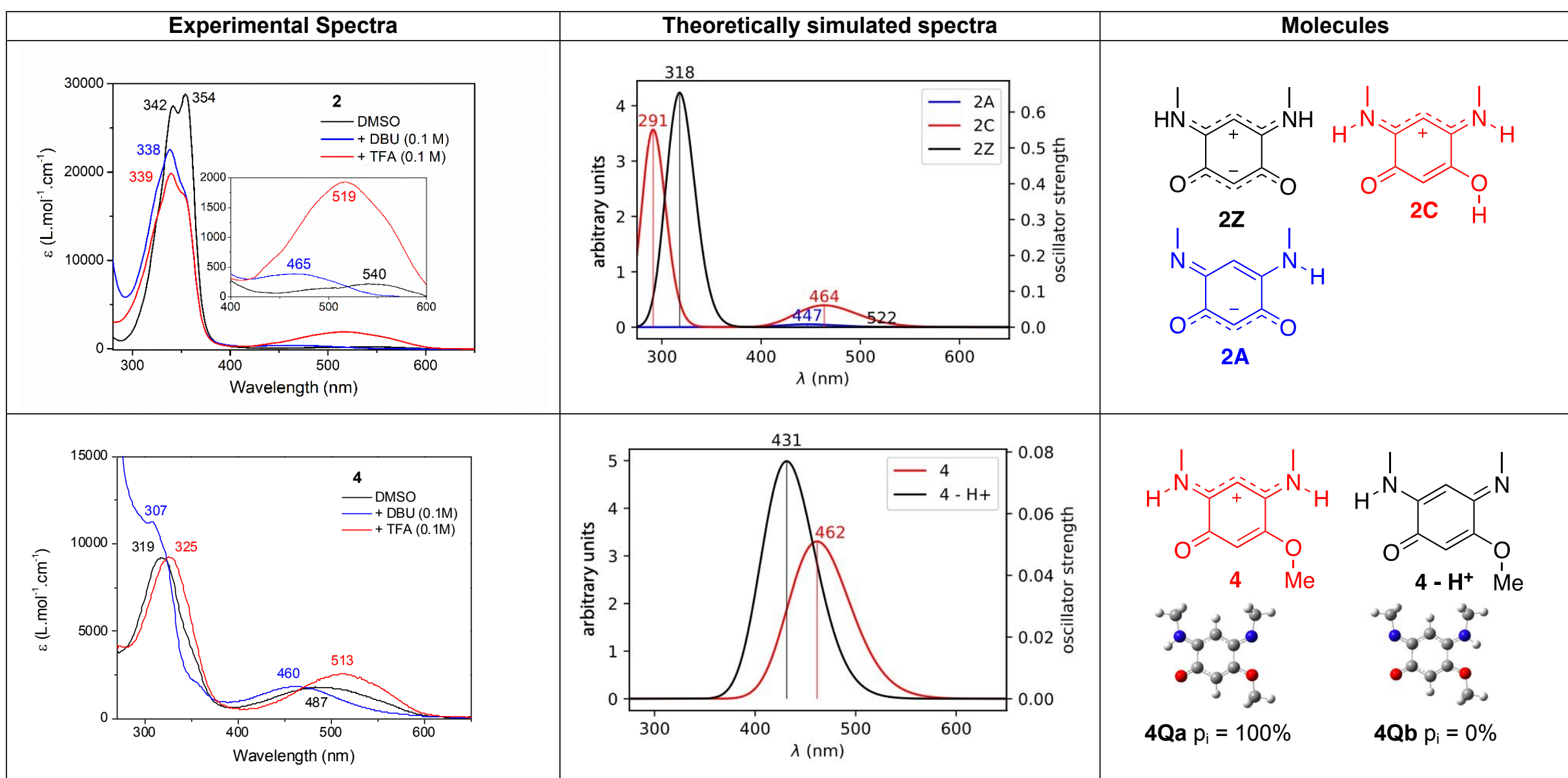

Figure S 48. (left) Experimental spectra of the compound $\mathbf{2}$ (top) and $\mathbf{4}$ (bottom) recorded in DMSO. (middle) Theoretical spectra obtained for the molecules 2, 2C, and 2A (top) and $\mathbf{4}$ and $\mathbf{4 Q a}$ (bottom) with a broadening Gaussian with $\sigma=0.25 \mathrm{eV}$. (right) schemes of the computed structures along with the $3 \mathrm{D}$ representation of the conformers for $\mathbf{4 Q}$ 


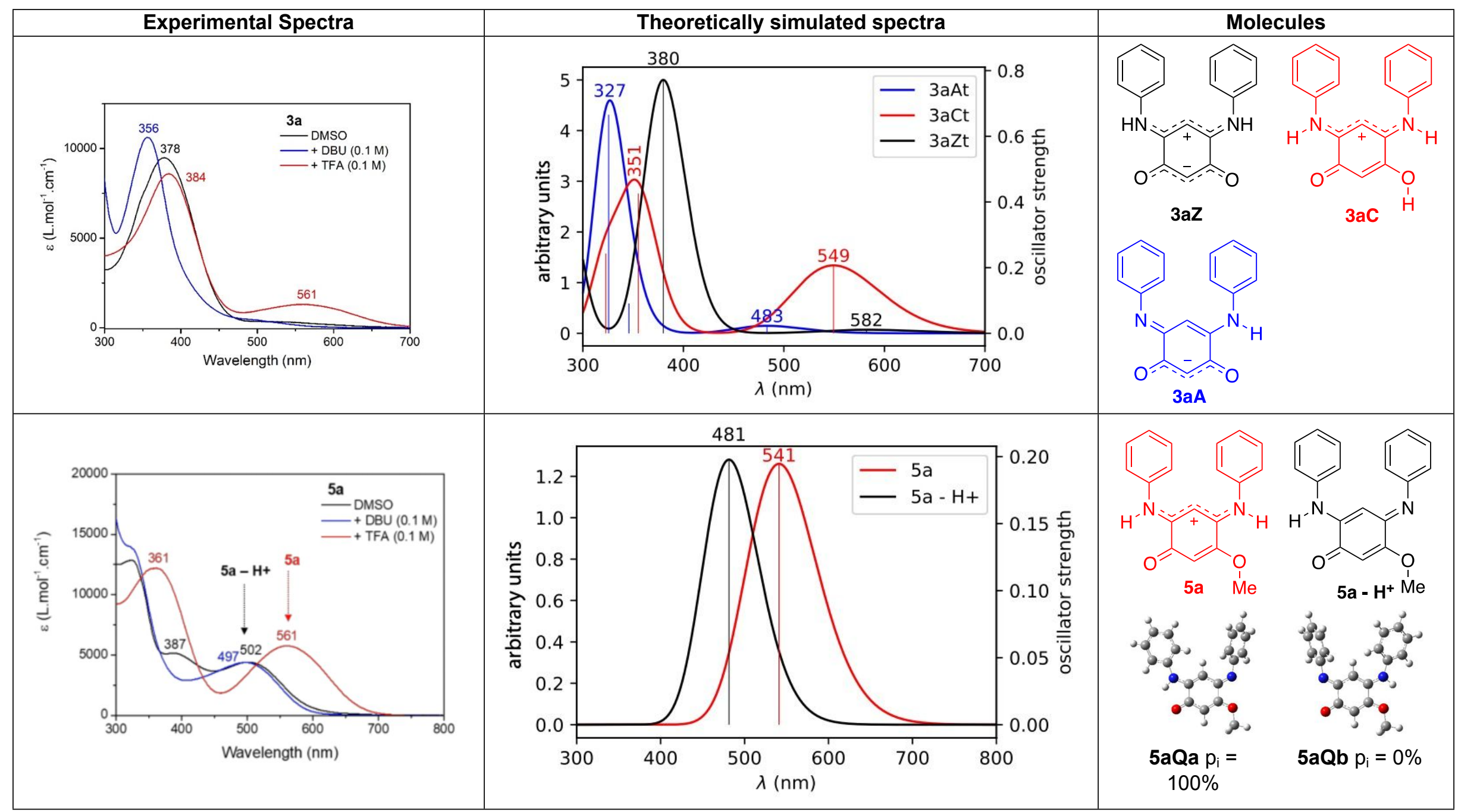

Figure S 49. (left) Experimental spectra of the compound 3a (top) and 5a (bottom) recorded in DMSO. (middle) Theoretical spectra obtained for the molecules 3aZt, 3aCt, and $\mathbf{3 a A t}$ (top) and $\mathbf{5 a}$ and $\mathbf{5 Q} \mathbf{a}$ (bottom) with a Gaussian broadening function with $\sigma=0.25 \mathrm{eV}$. (right) schemes of the computed structures along with the $3 \mathrm{D}$ representation of the conformers for $5 a Q$ 


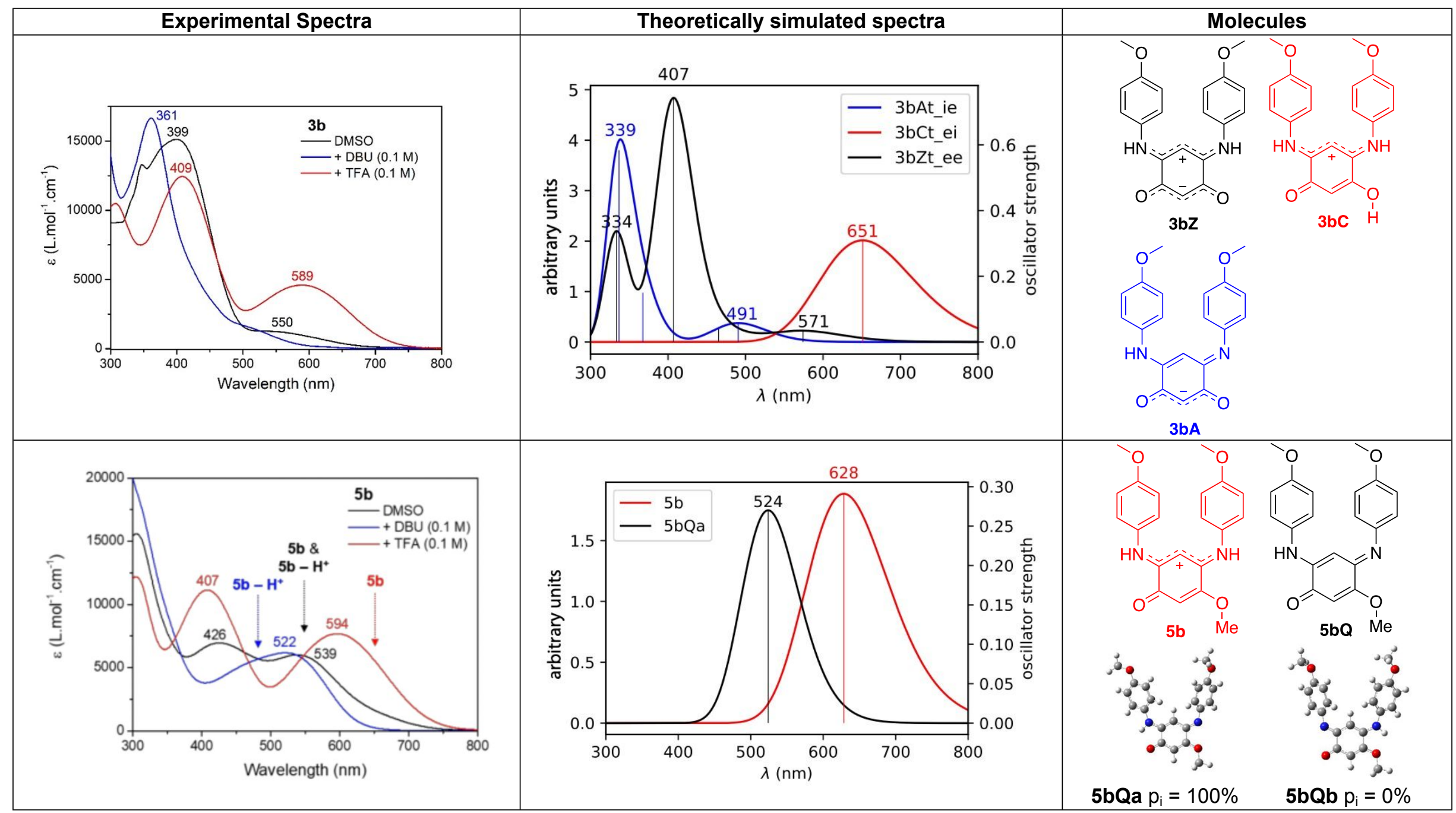

Figure S 50. (left) Experimental spectra of the compound $\mathbf{3 b}$ (top) and $\mathbf{5 b}$ (bottom) recorded in DMSO. (middle) Theoretical spectra obtained for the molecules $\mathbf{3 b Z t}$ ee, $\mathbf{3 b C t}$ ei, and $\mathbf{3 b A t}$ ie (top) and $\mathbf{5 b}$ and $\mathbf{5 b Q a}$ (bottom) with a Gaussian broadening function with $\sigma=0.25 \mathrm{eV}$. (right) schemes of the computed structures along with the 3D representation of the conformers for $\mathbf{5 b Q}$ 


\section{- Comparison of the nucleophilicity between $3 c$ and $3 d$ using conceptual DFT}

In order to explore the specific C-methylation happening in $\mathbf{3 d}$ versus the O-methylation observed in $3 \mathbf{b}$, we investigated the nucleophilicity of the $\mathrm{C}$ and $\mathrm{O}$ atoms of the negatively charged polymethine within the BQI core. To this end, we followed the principles of conceptual DFT, ${ }^{28}$ we calculated the change of partial atomic charges on C2 (central carbon) and 09 and 010 atoms when going from the neutral to a fictious positively charged compound. The greater the difference between $q($ neutral) and $q$ (radical cation), the greater the nucleophilicity of the corresponding atom. We tested several charge model (Table $S$ 10) however none of them was providing unequivocal results regarding the nucleophilicity of either the central carbon or one of the two oxygens. Given those results, it seems likely that nucleophilicity is not the only parameter playing a role in the different behavior of the two compounds.

Table S 10 Population analysis on compounds 3bZt_ee and 3dZt. Calculations carried at the M06-2X/aug-cc-pVTZ // B3LYP/Def2-TZVP level in DMSO(PCM).

\begin{tabular}{cccccccc}
\multirow{2}{*}{ Model } & Atom & $\mathbf{q}(\mathbf{0} \mathbf{1})$ & $\mathbf{q}(\mathbf{+ 1} \mathbf{2})$ & $\Delta(\mathbf{q})$ & $\mathbf{q}(\mathbf{0} \mathbf{1})$ & $\mathbf{q}(\mathbf{+ 1} \mathbf{2})$ & $\Delta(\mathbf{q})$ \\
\hline \multirow{3}{*}{ MK } & C2 & -0.705 & -0.584 & 0.120 & -0.794 & -0.779 & 0.015 \\
& O9 & -0.643 & -0.583 & 0.060 & -0.663 & -0.646 & 0.017 \\
& O10 & -0.643 & -0.583 & 0.060 & -0.665 & -0.649 & 0.016 \\
\hline \multirow{2}{*}{ Hirshfeld } & C2 & -0.147 & -0.090 & 0.057 & -0.149 & -0.140 & 0.009 \\
& O9 & -0.352 & -0.310 & 0.042 & -0.358 & -0.351 & 0.008 \\
& O10 & -0.352 & -0.310 & 0.042 & -0.359 & -0.351 & 0.008 \\
\hline \multirow{3}{*}{ NPA } & C2 & -0.496 & -0.137 & 0.359 & -0.484 & -0.236 & 0.248 \\
& O9 & -0.705 & -0.309 & 0.396 & -0.706 & -0.358 & 0.348 \\
& O10 & -0.698 & -0.308 & 0.390 & -0.706 & -0.357 & 0.349 \\
\hline \multirow{2}{*}{ NBO } & C2 & -0.496 & -0.137 & 0.359 & -0.484 & -0.474 & 0.011 \\
& O9 & -0.698 & -0.308 & 0.390 & -0.706 & -0.701 & 0.005 \\
& O10 & -0.705 & -0.309 & 0.396 & -0.706 & -0.699 & 0.006
\end{tabular}




\section{REFERENCES}

(1) Yang, Q.-Z.; Siri, O.; Braunstein, P. First transamination reactions for the one-pot synthesis of substituted zwitterionic quinones. Chem. Commun. 2005, 2660-2662.

(2) Tamboura, F. B.; Cazin, C. S. J.; Pattacini, R.; Braunstein, P. Reactions of Amines with Zwitterionic Quinoneimines: Synthesis of New Anionic and Zwitterionic Quinonoids. Eur. J. Org. Chem. 2009, 2009, 3340-3350.

(3) Dolomanov, O. V.; Bourhis, L. J.; Gildea, R. J.; Howard, J. A. K.; Puschmann, H. OLEX2: a complete structure solution, refinement and analysis program. J. Appl. Crystallogr. 2009, 42, 339-341.

(4) Sheldrick, G. SHELXT - Integrated space-group and crystal-structure determination. Acta Crystallogr. A 2015, 71, 3-8.

(5) Connelly, N. G.; Geiger, W. E. Chemical Redox Agents for Organometallic Chemistry. Chem. Rev. 1996, 96, 877-910.

(6) Gans, P.; O'Sullivan, B. GLEE, a new computer program for glass electrode calibration. Talanta 2000, 51, 33-37.

(7) Gampp, H.; Maeder, M.; Meyer, C. J.; Zuberbühler, A. D. Calculation of equilibrium constants from multiwavelength spectroscopic data-III: Model-free analysis of spectrophotometric and ESR titrations. Talanta 1985, 32, 1133-1139.

(8) Gampp, H.; Maeder, M.; Meyer, C. J.; Zuberbühler, A. D. Calculation of equilibrium constants from multiwavelength spectroscopic data-l: Mathematical considerations. Talanta 1985, 32, 95-101.

(9) Gampp, H.; Maeder, M.; Meyer, C. J.; Zuberbu"hler, A. D. Calculation of equilibrium constants from multiwavelength spectroscopic data-II132, 95.: Specfit: two user-friendly programs in basic and standard fortran 77. Talanta 1985, 32, 257-264.

(10) Marquardt, D. W. An Algorithm for Least-Squares Estimation of Nonlinear Parameters. J. Soc. Ind. Appl. Math. 1963, 11, 431-441.

(11) Maeder, M.; Zuberbuehler, A. D. Nonlinear least-squares fitting of multivariate absorption data. Anal. Chem. 1990, 62, 2220-2224.

(12) Frisch, M. J. et al., Gaussian 16, revision A.03p, Wallignford, CT, USA, 2016.

(13) TURBOMOLE V7.11: A development of University of Karlsruhe and Forschungszentrum Karlsruhe $\mathrm{GmbH}, 1989-2007$, TURBO-MOLE $\mathrm{GmbH}$, since 2007, available at: http://www.turbomole.com

(14) Hättig, C.; Weigend, F. CC2 excitation energy calculations on large molecules using the resolution of the identity approximation. 2000, 113, 5154-5161.

(15) Aidas, K.; Angeli, C.; Bak, K. L.; Bakken, V.; Bast, R.; Boman, L.; Christiansen, O.; Cimiraglia, R.; Coriani, S.; Dahle, P.; Dalskov, E. K.; Ekström, U.; Enevoldsen, T.; Eriksen, J. J.; Ettenhuber, P.; Fernández, B.; Ferrighi, L.; Fliegl, H.; Frediani, L.; Hald, K.; Halkier, A.; Hättig, C.; Heiberg, H.; Helgaker, T.; Hennum, A. C.; Hettema, H.; Hjertenæs, E.; Høst, S.; Høyvik, I.-M.; lozzi, M. F.; Jansík, B.; Jensen, H. J. A.; Jonsson, D.; Jørgensen, P.; Kauczor, J.; Kirpekar, S.; Kjærgaard, T.; Klopper, W.; Knecht, S.; Kobayashi, R.; Koch, H.; Kongsted, J.; Krapp, A.; Kristensen, K.; Ligabue, A.; Lutnæs, O. B.; Melo, J. I.; Mikkelsen, K. V.; Myhre, R. H.; Neiss, C.; Nielsen, C. B.; Norman, P.; Olsen, J.; Olsen, J. M. H.; Osted, A.; Packer, M. J.; Pawlowski, F.; Pedersen, T. B.; Provasi, P. F.; Reine, S.; Rinkevicius, Z.; Ruden, T. A.; Ruud, K.; Rybkin, V. V.; Sałek, P.; Samson, C. C. M.; de Merás, A. S.; Saue, T.; Sauer, S. P. A.; Schimmelpfennig, B.; Sneskov, K.; Steindal, A. H.; Sylvester-Hvid, K. O.; Taylor, P. R.; Teale, A. M.; Tellgren, E. I.; Tew, D. P.; Thorvaldsen, A. J.; Thøgersen, L.; Vahtras, O.; Watson, M. A.; Wilson, D. J. D.; Ziolkowski, M.; Ågren, H. The Dalton quantum chemistry program system. Comput. Mol. Sci. 2014, 4, 269-284.

(16) Guido, C. A.; Caprasecca, S. On the description of the environment polarization response to electronic transitions. 2019, 119, e25711.

(17) Cammi, R.; Mennucci, B. Linear response theory for the polarizable continuum model. J. Chem. Phys. 1999, 110, 9877-9886.

(18) Caricato, M.; Mennucci, B.; Tomasi, J.; Ingrosso, F.; Cammi, R.; Corni, S.; Scalmani, G. Formation and relaxation of excited states in solution: A new time dependent polarizable continuum model based on time dependent density functional theory. J. Chem. Phys. 2006, 124, 124520.

(19) Jacquemin, D.; Planchat, A.; Adamo, C.; Mennucci, B. TD-DFT Assessment of Functionals for Optical 0-0 Transitions in Solvated Dyes. J. Chem. Theory Comput. 2012, 8, 23592372.

(20) Adamo, C.; Barone, V. Toward reliable density functional methods without adjustable parameters: The PBE0 model. J. Chem. Phys. 1999, 110, 6158-6170. 
(21) Becke, A. D. Density-functional thermochemistry. III. The role of exact exchange. J. Chem. Phys. 1993, 98, 5648-5652.

(22) Zhao, Y.; Truhlar, D. The M06 suite of density functionals for main group thermochemistry, thermochemical kinetics, noncovalent interactions, excited states, and transition elements: two new functionals and systematic testing of four M06-class functionals and 12 other functionals. Theor. Chem. Acc. 2008, 120, 215-241.

(23) Yanai, T.; Tew, D. P.; Handy, N. C. A new hybrid exchange-correlation functional using the Coulomb-attenuating method (CAM-B3LYP). Chem. Phys. Lett. 2004, 393, 51-57.

(24) Chai, J.-D.; Head-Gordon, M. Long-range corrected hybrid density functionals with damped atom-atom dispersion corrections. Phys. Chem. Chem. Phys. 2008, 10, 6615-6620.

(25) Budzák, Š.; Scalmani, G.; Jacquemin, D. Accurate Excited-State Geometries: A CASPT2 and Coupled-Cluster Reference Database for Small Molecules. J. Chem. Theory Comput. 2017, 13, 6237-6252.

(26) Le Guennic, B.; Jacquemin, D. Taking Up the Cyanine Challenge with Quantum Tools. Acc. Chem. Res. 2015, 48, 530-537.

(27) Le Bahers, T.; Adamo, C.; Ciofini, I. A Qualitative Index of Spatial Extent in ChargeTransfer Excitations. J. Chem. Theory Comput. 2011, 7, 2498-2506.

(28) Geerlings, P.; De Proft, F.; Langenaeker, W. Conceptual Density Functional Theory. Chem. Rev. 2003, 103, 1793-1874. 